\title{
Interactive Bayesian Identification of Kinematic \\ Mechanisms
}

by

\author{
Patrick Rene Barragán
}

Submitted to the Department of Mechanical Engineering in partial fulfillment of the requirements for the degree of

Doctor of Philosophy in Mechanical Engineering at the

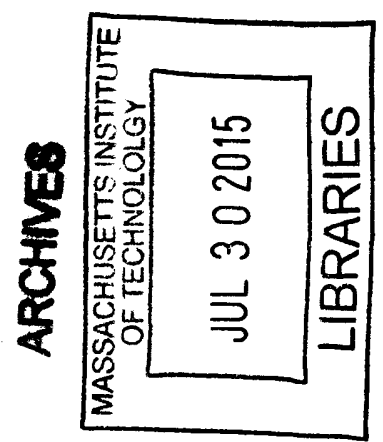

MASSACHUSETTS INSTITUTE OF TECHNOLOGY

June 2015

(c) Massachusetts Institute of Technology 2015. All rights reserved.

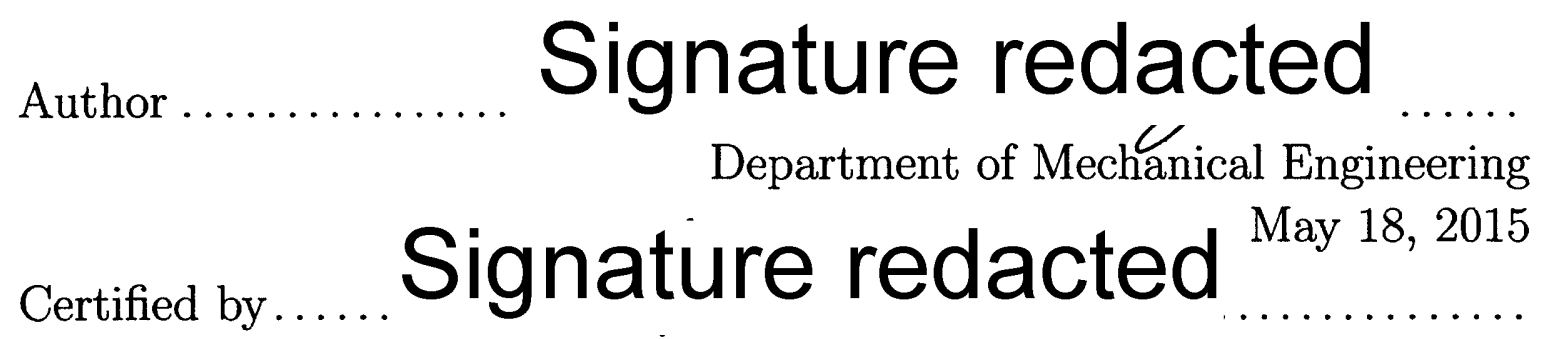

Leslie Pack Kaelbling Professor of Computer Science and Engineering Certified by.... Signäture redacted ${ }_{\text {Tómás Lozano-Pérez }}^{\text {Thesis Supervisor }}$ Professor of Computer Science and Engineering

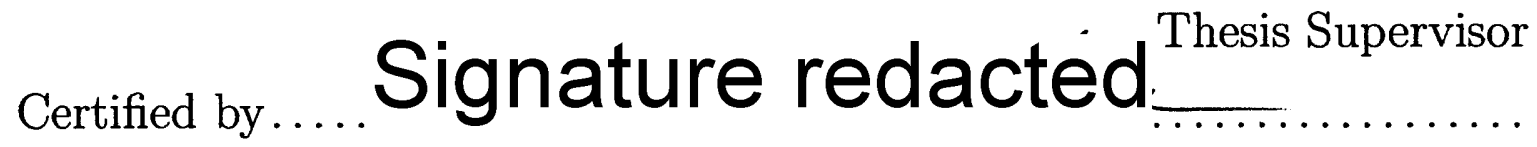
Franz S. Hover Associate Professor of Mechanical Engineering Than Thesis Committee Accepted by ..... Signature redacted

David E. Hardt Chairman, Department Committee on Graduate Students 



\title{
Interactive Bayesian Identification of Kinematic Mechanisms
}

\author{
by \\ Patrick Rene Barragán \\ Submitted to the Department of Mechanical Engineering \\ on May 18, 2015, in partial fulfillment of the \\ requirements for the degree of \\ Doctor of Philosophy in Mechanical Engineering
}

\begin{abstract}
This thesis addresses the problem of identifying mechanisms based on data gathered from a robot's interaction with them. We present a decision-theoretic formulation of this problem, using Bayesian filtering techniques to maintain a distributional estimate of the mechanism type and parameters.

We begin by implementing a discrete Bayesian filter. We demonstrate the approach on a domain with four primitive and two composite mechanisms. In order to reduce the amount of interaction required to arrive at a confident identification, we select actions explicitly to either a) reduce entropy in the current estimate or b) race the top two hypotheses to clearly distinguish between them. The results show that this approach can correctly identify complex mechanisms including mechanisms which behave different in different parts of their configuration spaces. The results also show that active action selection can significantly decrease the number of actions required to gather the same information while the racing technique does so without increasing step time of the filter over random action selection.

We analyze Bayesian filtering in a hybrid space for model comparison. We discuss the appropriateness of continuous state-space, parametric filters for the mechanism identification problem. We seek an alternative strategy because no parametric form is clearly suited to representing the posterior distributions during the filtering process. We find that Bayesian filtering in the hybrid space has some surprising consequences and discuss their effect on inference.

Finally, we implement a particle filter which allows filtering in a space expanded from 10 model-parameter pairs to 50,000. We demonstrate that with high accuracy, the particle filter can correctly identify the mechanism type. More crucially, we show that the filter's estimate allows the robot to reasonably predict the motion of the given mechanism regardless of classification. We demonstrate our method in simulation and on a real-world PR2 robot interacting with common mechanisms.
\end{abstract}

Thesis Supervisor: Leslie Pack Kaelbling

Title: Professor of Computer Science and Engineering

Thesis Supervisor: Tomás Lozano-Pérez

Title: Professor of Computer Science and Engineering 


\section{Acknowledgments}

First, I would like to thank the Lord Jesus Christ without whom nothing would be possible. Next, I would like to thank my entire family, near or far. Without all of the sacrifices my family has made for me over my entire life, I never would have had any the opportunities I have been so blessed to have. Just two generations ago, my grandparents struggled to feed their families. Now, I have the opportunity to acknowledge them in a doctoral thesis. I need to thank my father's parents Mauricio and Rebecca Barragán and my mother's parents Florentino and Zoraida Couce. I owe them immeasurably for struggling through decades of hardship that would never be rewarded in their lifetimes. I give thanks to them for never giving up and thanks to them for creating all of the opportunities that our family will ever see. I owe extra thanks to mi abuela Zorida for helping raise me. I learned so much from her that I will never forget. I will make sure to pass on those lessons to as many people as I can. She went to the Lord while I was a graduate student. Even when she was the most sick, she always told me to return to school, work hard, and finish my doctorate so that my family could have a better life. Abuela, I finished. I would like to thank my brother Blas Jr. for teaching me to analyze the world. He has always encouraged me to push for my best. I would not have accomplished a fraction as much as I have without him. Finally, I would like to thank my loving parents Blas Sr. and Maria. They have sacrificed their entire lives to put my brother and I where we are today. They always made our education top priority over anything else including any luxury or comfort they could have ever had. No words can express my thanks to them. I only hope they know they are the only reason I am here.

I would also like to thank the Learning and Intelligent Systems laboratory starting with Professor Leslie Pack Kaelbling and Professor Tomás Lozano-Pérez, my thesis advisors. They taught me how to be a researcher. They inspired a passion and excitement for research that is invaluable to me. Yet, they were more concerned with how I was and what I was learning than anything else. I cannot properly describe how they have changed the course of my life. I could not imagine having a better 
set of advisors. I would like to thank all of my lab-mates. Special thanks goes to Ariel Anders, Clement Gehring, Gustavo Goretkin, and Owen MacIndoe for all of the countless hours of your time spent helping me and discussing my thesis problem. Extra special thanks is owed to Jennifer Barry and Lawson Wong. Jenny helped me on my very first day in lab and never stopped. She guided me through a difficult acclimation to this field. Finally, I would have not completed this thesis without Lawson's guidance, knowledge, and friendship. He has helped me in nearly ever way imaginable. The strength of my work owes a great deal to him. If for nothing else, I am glad to have joined this lab for the amount of time I have been able to spend with him.

I would like to thank all of my professors, teachers, lab instructors, coaches, and colleagues from the beginning until now. I would like to thank my committee members Professor Franz Hover and Professor Alberto Rodriguez for their help in this process. I would like to specially thank Professor Seth Teller. He was one of the first people to get me excited about robotics. I miss him. The people who have taught me and helped me learn and become a better person are innumerable. Without them, I would have not made it here. I owe them more than I could ever repay. I owe special thanks to Halston W. Taylor, the director of the MIT track and field team. He has guided and supported me through my entire career at MIT. I owe him for helping me become the person I am today.

I would like to thank all of the groups that I am a part of at MIT including the gymnastics team, the sailing team, the track and field team, the MIT glass lab, and East Campus 1st West for the endless fun and enjoyment I have had. I would like to especially thank the pole vaulters with whom I spend the most time. Being part of MIT pole vault has been one of the most rewarding experiences I have had in my time here.

I would like to thank my countless friends for keeping me sane and supporting me everyday. The number of people to whom I owe thanks would not fit on these pages. I hope they all know how important they are to me. In a special way, I would like to thank James Albrecht. His gifts and his friendship cannot be replaced. I miss him 
dearly. I would also like to thank Lindsay Sanneman. She has supported me through the toughest years of my doctorate. I owe her so much. In a very special way, I need to thank Billy Putnam. Billy and I met at age four. We have gone to the same schools ever since, including doctoral programs at MIT, and have never lived more than a mile or so apart. He encouraged me many times to try something I was unsure I could accomplish. Without his encouragement and friendship, I would have never attempted so many things that have led me to today. I hope that we have many more years of friendship to come.

And finally, to Dave Wottle, I made it one last time. 


\section{Contents}

1 Introduction $\quad 21$

1.1 Problem Formulation . . . . . . . . . . . . . . . . . . 22

1.1.1 Model Specification ................. 22

1.1.2 Bayesian Inference . . . . . . . . . . 25

1.1.3 Recursive Bayesian Filtering . . . . . . . . . . . . . 26

1.2 Rationale .......................... 27

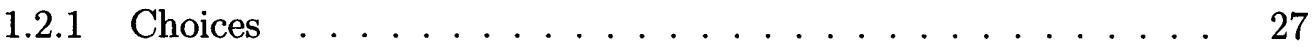

1.2.2 Requirements for a New Problem . . . . . . . . . . . . 29

1.3 Contributions . . . . . . . . . . . . . . . . . 29

1.4 Thesis Organization . . . . . . . . . . . . . . . 30

2 Related Work $\quad 31$

2.1 Vision-based Techniques ................. . . 31

2.2 Gripper-based Measurements . . . . . . . . . . . . . . . 33

2.3 Action Selection . . . . . . . . . . . . . . . . 34

2.4 Environment Exploration . . . . . . . . . . . . . . . 35

2.5 Our Approach . . . . . . . . . . . . . . . . 36

3 Discrete Filter $\quad 39$

3.1 Discrete Bayesian Inference _ . . . . . . . . . . . . . . . . . 40

3.2 Experimental Domain and Implementation . . . . . . . . . . 41

3.2 .1 States ........................ 41

3.2 .2 Actions ........................ . . 44 


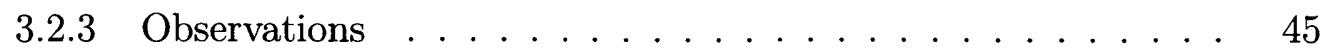

3.2.4 Transition and Observation Models . . . . . . . . . . . 45

3.2.5 Simulated Robot and "True" Mechanism . . . . . . . . . . 47

3.2 .6 Experimental Details . . . . . . . . . . . . . . . . . 48

3.3 Active Exploration $\ldots \ldots \ldots \ldots \ldots \ldots \ldots$

3.4 Critical Computations . . . . . . . . . . . . . . . 50

3.5 Results . . . . . . . . . . . . . . . . . . . 51

3.5 .1 Single Trial . . . . . . . . . . . . . . . . . 52

3.5 .2 Full Trials $\ldots \ldots \ldots \ldots \ldots \ldots \ldots \ldots$

3.6 Expanding the Space $\ldots \ldots \ldots \ldots \ldots \ldots \ldots$

$3.6 .1 \quad$ Expanded State Space $\ldots \ldots \ldots$. . . . . . . . . 58

$3.6 .2 \quad$ Statistical Racing . . . . . . . . . . . . . . . . 58

3.6 .3 Results . . . . . . . . . . . . . . . . . . 61

3.7 Discussion . . . . . . . . . . . . . . . . . . . . 68

$3.7 .1 \quad$ Limitations $\ldots \ldots \ldots \ldots \ldots$

4 Consequences of Bayesian Filtering for Hypothesis Comparison $\quad 71$

4.1 Continuous State-space, Parametric Filters . . . . . . . . . . . 71

4.2 Unintuitive Results of Bayesian Filtering in a Hybrid Space . . . . . 74

4.2.1 Transition Model Quality . . . . . . . . . . . 74

$4.2 .2 \quad$ Bayesian Occam's Razor . . . . . . . . . . . . . . 77

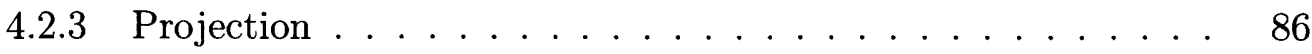

5 Particle Filter $\quad 93$

5.1 Particle Filter Implementation . . . . . . . . . . . . . . . . 94

5.2 Specific Assumptions for the Given Problem . . . . . . . . . 96

5.2.1 Bank of Particle Filters . . . . . . . . . . . . . . 96

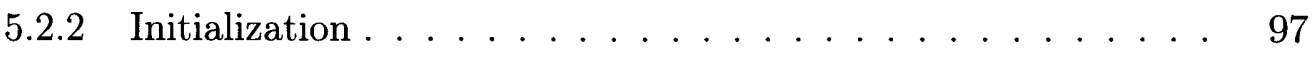

5.3 Model and Filter Parameterization _ . . . . . . . . . . . . . . . 99

5.3.1 Transition and Observation Model Covariances . . . . . . . . 99

5.3 .2 Number of Particles . . . . . . . . . . . . . . . . . . . 102 
5.4 Results ......................... 104

5.4 .1 Evaulation Metric . . . . . . . . . . . . . . . 104

5.4 .2 Simulation Results . . . . . . . . . . . . . . 112

5.4 .3 Real-robot Results . . . . . . . . . . . . . . 115

5.4 .4 Limitations . . . . . . . . . . . . . . . . 118

6 Conclusion $\quad 119$

6.1 Reflections ....................... 119

6.2 Summary of Contributions . . . . . . . . . . . . . . . . 122

6.3 Future Work . . . . . . . . . . . . . . . . . . . 123 


\section{List of Figures}

1-1 Willow Garage PR2 robot manipulating revolute model . . . . . . . . 23

3-1 Diagrams of the each of the six models considered. Fixed parameters are shown in red while variables are shown in blue. The large dot represents each mechanism's handle. . . . . . . . . . . . .

3-2 a) The robot's absolute actions are attempted moves from any point in the workspace to one of the designated end points. The workspace is denoted as the dashed, black line. The end target points are shown as black dots with red outlines. Absolute actions are used in the experiments discussed in Section 3.5. b) The robot's relative actions are attempted moves from the gripper's current pose in one of eight directions. The start pose is denoted as a black dot with green outline. The end target points are shown as black dots with red outlines. Relative actions are used in the experiments discussed in Section 3.6.3. . . . .

3-3 Single trial for instance of the Latch 2 model type using entropy-based action selection. . . . . . . . . . . . . . . . 52

3-4 Filter convergence and random vs. entropy-based action selection from free, fixed, and revolute models. . . . . . . . . . . . . . .

3-5 Filter convergence and random vs. entropy-based action selection from prismatic, Latch 1 , and Latch 2 models. . . . . . . . . . 56

3-6 Filter convergence and random vs. entropy-based vs. statistical racing action selection from free and fixed models. . . . . . . . . . . . . 
3-7 Filter convergence and random vs. entropy-based vs. statistical racing action selection from Revolute 1 and Prismatic 1 models. . . . . . . .

3-8 Filter convergence and random vs. entropy-based vs. statistical racing action selection from Latch 11 and Latch 21 models. . . . . . . . . .

3-9 Filter convergence and random vs. entropy-based vs. statistical racing action selection from Revolute 2 and Prismatic 2 models. . . . . . . .

3-10 Filter convergence and random vs. entropy-based vs. statistical racing action selection from Latch 12 and Latch 22 models. . . . . . . . . .

4-1 The mechanism identification problem is presented as a hybrid state space. The space is discrete over mechanism types, and each mechanism type has an associated, possibly different-dimensionality subspace. Regardless of mechanism type, a state, shown as a green dot, maps to a nominal observation, show as magenta dot, in the observation space. . . . . . . . . . . . . . . .

4-2 Comparison of the effect of model quality on the observation likelihood ratio between a revolute and prismatic hypothesis tangent to the revolute hypothesis at the origin. These hypotheses act similarly in this region of the observation space. All possible noiseless, nominal observations of each mechanism are represented as a labeled blue line. The start state at the origin of the observation space is shown as a black dot with green outline. The action taken is denoted as a black arrow. The end states in the observation space assuming each type are shown as black dots with red outlines. The observation is shown as a black dot with magenta outline. a) The filter models of the mechanisms are perfect b) The filter has a good quality model of the prismatic mechanism but a poor quality model of the revolute mechanism. . . . . . . . . . 
4-3 Visual depiction of the Bayesian Occam's razor phenomenon for a scenario of comparing a free hypothesis and a prismatic hypothesis given an observation close to $y=0$. Blue denotes regions of lower probability, while red denotes regions of higher probability. The black circles and black line denote regions where states or their corresponding nominal observations would assign nonzero probability to observation $o$. a) $P(\Theta \mid M)$ shown on the configuration space of the free model. b) $P(\Theta \mid M)$ shown on the configuration space of the prismatic model. c) $P\left(O^{*} \mid M\right)$ shown on the observation space of the free model. d) $P\left(O^{*} \mid M\right)$ shown on the observation space of the prismatic model. . .

4-4 Visual depiction of the Bayesian Occam's razor phenomenon for a scenario of comparing a free hypothesis and a prismatic hypothesis given an observation far from $y=0$. Blue denotes regions of lower probability, while red denotes regions of higher probability. The black circles and black line denote regions where states or their corresponding nominal observations would assign nonzero probability to observation $o$. a) $P(\Theta \mid M)$ shown on the configuration space of the free model. b) $P(\Theta \mid M)$ shown on the configuration space of the prismatic model. c) $P\left(O^{*} \mid M\right)$ shown on the observation space of the free model. d) $P\left(O^{*} \mid M\right)$ shown on the observation space of the prismatic model. . . 
4-5 Visual depiction of the projection phenomenon for a scenario of comparing a free hypothesis and a scaled free hypothesis given an observation close to the origin of the observation space. The darkest blue denotes regions of zero probability. The lighter blue denotes regions of lower probability, while orange denotes regions of higher probability. The black circles denote regions where states or their corresponding nominal observations would assign nonzero probability to observation o. a) $P(\Theta \mid M)$ shown on the configuration space of the free model. b) $P(\Theta \mid M)$ shown on the configuration space of the scaled free model. c) $P\left(O^{*} \mid M\right)$ shown on the observation space of the free model. d) $P\left(O^{*} \mid M\right)$ shown on the observation space of the scaled free model. .

4-6 Visual depiction of the projection phenomenon for a scenario of comparing a free hypothesis and a scaled free hypothesis given an observation far from the origin of the observation space. The darkest blue denotes regions of zero probability. The lighter blue denotes regions of lower probability, while orange denotes regions of higher probability. The black circles denote regions where states or their corresponding nominal observations would assign nonzero probability to observation o. a) $P(\Theta \mid M)$ shown on the configuration space of the free model. b) $P(\Theta \mid M)$ shown on the configuration space of the scaled free model. c) $P\left(O^{*} \mid M\right)$ shown on the observation space of the free model. d) $P\left(O^{*} \mid M\right)$ shown on the observation space of the scaled free model. .

5-1 The mean-squared error after 10 actions between the true state and best particle in the filter for varying number of particles per model. . 103 
5-2 The ability to predict the true mechanisms motion may be a more applicable evaluation metric for the mechanism identification problem than misclassification. In this top-down view, the robot's workspace is denoted by a dashed box centered on the origin of the observation space denoted by the axes. A true revolute mechanism is shown by the straight green line with the mechanism's handle at the origin of the observation space. The light green circle shows the locus of nominal observations for this mechanism. The straight red line denotes a properly-classified revolute hypothesis with a corresponding nominalobservation locus shown as a light red circle. The light blue line denotes the nominal-observation locus, tangent to the nominal-observation locus of the true mechanism at the origin, of an improperly-classified prismatic hypothesis. In this example, the prismatic hypothesis would better predict the true mechanisms motions in the workspace. . . . 106

5-3 Sequence of actions used in the distance metric in the robot workspace $(x, y)$ viewed from above. The action sequence is 24 actions where the first action is denoted by the lightest blue color and the last action is denoted by the brightest pink color. The sequence starts at $(0,0)$, moves in the positive $x$ direction, then in the negative $y$ direction, and continues clockwise around the workspace. . . . . . . . . 108 
5-4 Top-down view of the sequence of observations that was generate by the same action sequence, shown in Figure 5-3, on both a true revolute joint, drawn in green, and an estimated revolute joint. The legal configuration space projected into the observation space of the true mechanism is drawn as a green circle, and that of the estimated mechanism is drawn as a red circle. Shown as points, the observations are colored the same as the corresponding actions in the previous figure and drawn on the corresponding mechanism. The dashed blue lines connect corresponding observations and show the distance of the prediction error. The average of these distances is used as the quality metric of the estimate. The average distance between nominal observations for these two mechanisms and initial states is $7[\mathrm{~cm}] . \ldots 110$

5-5 Top-down view of the sequence of observations that was generate by the same action sequence, shown in Figure 5-3, on both a true revolute joint and an estimated prismatic joint that is tangent to the true revolute joint at the origin. The legal configuration space projected into the observation space of the true mechanism is drawn as a green circle, and that of the estimated mechanism is drawn as a red line. The figure is drawn in the same manner as Figure 5-4. The average distance between nominal observations for these two mechanisms and initial states is 6 $[\mathrm{cm}] \ldots \ldots \ldots \ldots \ldots \ldots \ldots$ 


\section{List of Tables}

3.1 Space of possible mechanism states $\ldots \ldots \ldots \ldots \ldots \ldots$

3.2 Experimental parameter values for mechanism types . . . . . . . 48

3.3 Experimental parameter values for mechanism types . . . . . . . 58

3.4 Averaged over 100 experiments per action selection type, the probability of most likely model-parameter pair after five actions and after ten actions for the random, entropy-minimizing, and statistical racing action-selection techniques. . . . . . . . . . . . . . . .

3.5 Averaged over 100 experiments per action selection type, the running time of a step of the filter for the random, entropy-minimizing, and statistical racing action-selection techniques. . . . . . . . . . .

3.6 Out of 100 experiments per action selection type, the number of misclassification errors for the random, entropy-minimizing, and statistical racing action-selection techniques. . . . . . . . . . . .

5.1 Total misclassifications in simulation experiments out of 50 total experiments. . . . . . . . . . . . . . . . . 113

5.2 Confusion matrix for misclassifications in simulation experiments out of 50 total experiments. . . . . . . . . . . . . . . . . . . 114

5.3 Error and standard deviation (in $[\mathrm{cm}]$ ) of state estimate using the action sequence evaluation metric over models averaged over 50 simulation experiments without including misclassified estimates. . . . . . 114 
5.4 Error and standard deviation of state estimate using the action sequence evaluation metric over models averaged over 50 simulation experiments including misclassified estimates. . . . . . . . . . . . . 115

5.5 Total misclassifications in simulation experiments out of 50 total experiments. . . . . . . . . . . . . . 116

5.6 Confusion matrix for misclassifications in robot experiments out of 10 total experiments. . . . . . . . . . . . . . . . 117

5.7 Error and standard deviation of state estimate using the action sequence evaluation metric over models averaged over 10 robot experiments without including misclassified estimates. . . . . . . . . . . 117

5.8 Error and standard deviation of state estimate using the action sequence evaluation metric over models averaged over 10 robot experiments including misclassified estimates. . . . . . . . . . . . . 118 


\section{Chapter 1}

\section{Introduction}

Consider a household robot that can move and grasp objects. It arrives in a new house and must quickly learn to interact with a variety of kinematic mechanisms: cupboard doors that rotate about hinges on the left or right or that slide sideways; drawers that pull out; security latches on the front door; faucet handles that rotate or slide along multiple axes.

We would expect the robot already to know about a general class of such mechanisms learned from previous experience, given by other robot experience, or described appropriately by a human. Then, faced with a new object, the robot would like to be able to grasp and attempt to move it, possibly receiving information from several modalities, including joint torques and positions, tactile feedback, and visual tracking of parts of the object. In this process, the robot should quickly be able to discover the type of mechanism it is interacting with, as well as its parameters, such as hinge location, radius of rotation, etc.

In this thesis, we present a decision-theoretic formulation of this problem, using Bayesian filtering techniques to maintain a distributional estimate of the mechanism's type and parameters. In order to reduce the amount of interaction required to arrive at a confident identification, we also consider selecting actions explicitly to reduce entropy in the current estimate.

Instead of identifying the mechanism, if the ultimate goal of the robot is to open a cupboard door or to cause water to come out of faucet, then this problem is appro- 
priately formulated as a partially-observable Markov decision process (POMDP) [19]. Such a formulation would support optimal action exploration in service of achieving the goal. Solution of POMDPs can be computationally very difficult, so in this work, we focus on the goal of identifying the mechanism, including its parameters, and use a greedy action-selection method.

We apply this framework in a simple experimental domain with both primitive and composite mechanisms and demonstrate in simulation and on a real robot that the system can use position information to perform effectively and that informationdriven action selection offers significant advantages. We have conducted experiments on a PR2 robot, using active exploration and position information to discriminate among the mechanisms.

We applied several different forms of Bayesian filters to the problem and explored their strengths and weaknesses. The success and limitations of the discretized filter show the potential of filtering but the need for faster methods. We apply particle filtering to the problem and explore the intricacies of particle filtering in a hybrid state space as well as its limitations. Overall, we demonstrate the effectiveness of Bayesian filtering to address the identification and estimation of every-day kinematic mechanisms.

\subsection{Problem Formulation}

We formulate the process of interacting with the mechanism as a discrete-state, InputOutput Hidden Markov Model (IOHMM), in which the hidden states are the states of the mechanisms, inputs are actions taken by the robot, and outputs are the sensed data.

\subsubsection{Model Specification}

The state of a kinematic system is a tuple $S=\langle m, \theta, x\rangle$, where:

- $m$ is the type of the mechanism. The type specifies a particular class of the object. These types need not simply be serial linkages. In general, a model 


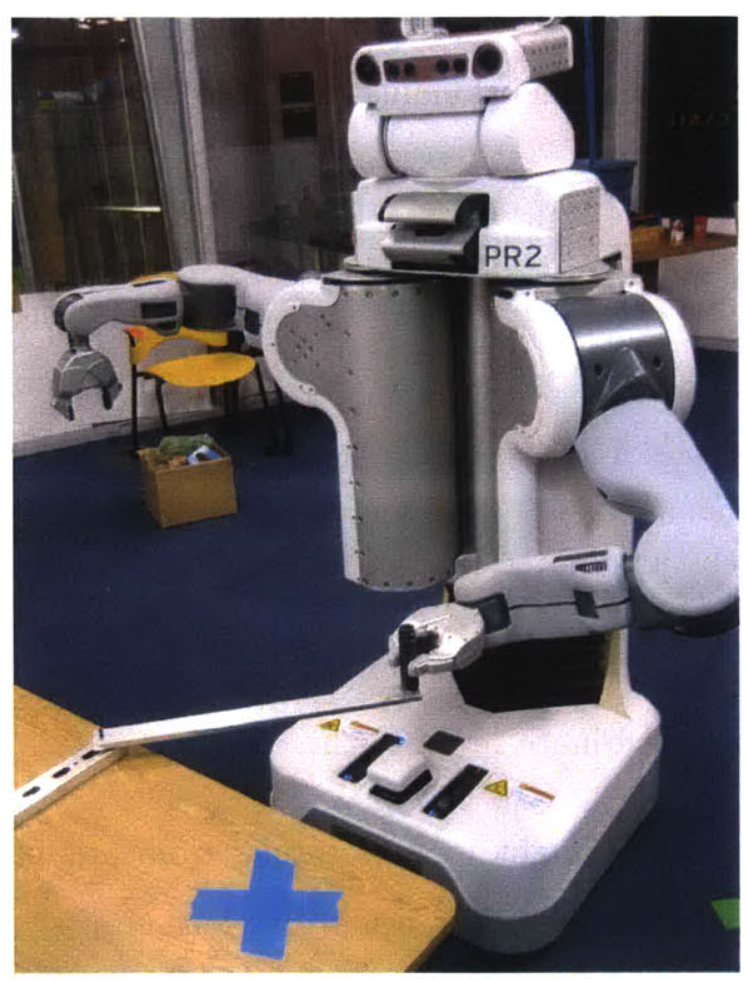

Figure 1-1: Willow Garage PR2 robot manipulating revolute model

class could describe a wide range of possible objects (e.g. soft bodies or systems of objects). In this work, we will focus on kinematic mechanisms. These objects can have any number of degrees of freedom, including "sequential" mechanisms, such as latches, in which some degrees of freedom may only be accessible when others are in a specific range of configurations. In this work, we will enumerate a finite set of types in advance.

- $\theta$ is a vector of parameters of the mechanism. Objects of a given type $m$ will be characterized by a finite set of (typically continuous) parameters. Examples would be the position of the pivot and radius of a revolute joint or axis specifications of a prismatic joint.

- $x$ is a vector of variables that specify a particular current configuration of the mechanism. Examples would be the current angle of a revolute joint or Cartesian position of a free mechanism. 
For the purposes of identifying a mechanism, the state space will consist of a set of $\langle m, \theta, x\rangle$ tuples, with the dimensionality of $\theta$ and $x$ depending on the particular type $m$.

The action space $A$ might include any set of primitive actions a robot could take to get information about or change the state of the mechanism it is interacting with, including looking at it from different viewpoints, pushing, pulling, etc. The only requirements on the space of actions are:

- Any action may be attempted on any mechanism type with any parameter and variable values.

- All actions always terminate in finite time.

The observation space $O$ might include inputs from any of the robot's sensors, including vision, tactile, force, position, and vibration sensors.

Once the spaces are defined, we must characterize their relationships. The transition model describes the effects of actions on the state of the system as a conditional probability distribution over values of the state at time $t+1$ given the state at time $t$ and the action executed at time $t$. This is a discrete-time model that assumes each primitive action is run to completion. We can factor the transition model into two components: the probability that the mechanism type or parameters change (for example, because the robot breaks a constraint on the mechanism, or because the mechanism becomes jammed) and the probability that the variables change, given the mechanism type and parameters.

$$
\begin{aligned}
\operatorname{Pr}\left(s_{t+1} \mid s_{t}, a_{t}\right)= & \operatorname{Pr}\left(m_{t+1}, \theta_{t+1}, x_{t+1} \mid m_{t}, \theta_{t}, x_{t}, a_{t}\right) \\
= & \operatorname{Pr}\left(m_{t+1}, \theta_{t+1} \mid m_{t}, \theta_{t}, x_{t}, a_{t}\right) \\
& \cdot \operatorname{Pr}\left(x_{t+1} \mid m_{t+1}, \theta_{t+1}, x_{t}, a_{t}\right)
\end{aligned}
$$

The characterization of type and parameter change,

$$
\operatorname{Pr}\left(m_{t+1}, \theta_{t+1} \mid m_{t}, \theta_{t}, x_{t}, a_{t}\right)
$$


might be written by hand or learned from very long-term interactions. We expect that, for now, it will be sufficient to use a distribution that keeps $m$ and $\theta$ at their previous values with high probability and offers a near uniform change to other values. The typical operation of the mechanism is characterized by

$$
\operatorname{Pr}\left(x_{t+1} \mid m_{t+1}, \theta_{t+1}, x_{t}, a_{t}\right)
$$

which is a model of how the variables of a mechanism of type $m$ with parameters $\theta$ change in response to actions of the robot. Such models may be described analytically, through the use of forward simulation, or estimated from data.

The observation model specifies a distribution over elements of $O$, given the current state and previous action:

$$
\operatorname{Pr}\left(o_{t+1} \mid m_{t+1}, \theta_{t+1}, x_{t+1}, a_{t}\right)
$$

Exactly how this model is to be specified depends on the sensors involved. Note that $o$ can be a vector of observations from different sensing modalities.

\subsubsection{Bayesian Inference}

We will focus on the problem of identifying the type and parameters of the mechanism that the robot is interacting with. Having made such an identification, the robot would then be able to plan to manipulate the mechanism to achieve multiple goals within the operating space of the mechanism, such as opening or closing a door.

Given a sequence of actions, $a_{0: T-1}=a_{0}, \ldots, a_{T-1}$, and observations made as a result of those actions, $o_{1: T}=o_{1}, \ldots, o_{T}$, as well as a distribution characterizing the a priori belief in the different elements of the state space, $\operatorname{Pr}\left(s_{0}\right)$, we are interested in computing the posterior distribution over types and parameters, which is obtained 
by marginalizing out the variables at time $T$. Explicitly,

$$
\begin{aligned}
\operatorname{Pr}\left(m_{T}, \theta_{T} \mid a_{0: T-1}, o_{1: T}\right) & =\int_{x_{T}} \operatorname{Pr}\left(m_{T}, \theta_{T}, x_{T} \mid a_{0: T-1}, o_{1: T}\right) d x_{T} \\
& =\int_{x_{T}} \operatorname{Pr}\left(s_{T} \mid a_{0: T-1}, o_{1: T}\right) d x_{T} .
\end{aligned}
$$

This expression can be further seen as a marginalization over the states of the mechanism from times 0 through $T-1$ :

$$
\int_{x_{T}} \int_{s_{0: T-1}} \operatorname{Pr}\left(s_{0: T} \mid a_{0: T-1}, o_{1: T}\right) d s_{0: T-1} d x_{T}
$$

Then, we can use Bayes' rule and the conditional independence relationships in an IOHMM to write

$$
\begin{aligned}
& \operatorname{Pr}\left(m_{T}, \theta_{T} \mid a_{0: T-1}, o_{1: T}\right) \propto \\
& \qquad \int_{x_{T}} \int_{s_{0: T-1}} \operatorname{Pr}\left(s_{0}\right) \prod_{t=0}^{T-1} \operatorname{Pr}\left(o_{t+1} \mid s_{t+1}, a_{t}\right) \operatorname{Pr}\left(s_{t+1} \mid s_{t}, a_{t}\right) d s_{0: T-1} d x_{T} .
\end{aligned}
$$

Depending on particular representational choices, this quantity can typically be computed efficiently using dynamic programming or recursive filtering.

\subsubsection{Recursive Bayesian Filtering}

In this thesis, we choose to compute the posterior distribution by recursive Bayesian filtering. Filtering provides a distribution on the hypotheses at each step instead of using batch data over a number of steps as some fitting-based methods must. Relying on the Markov assumption, recursive filtering consists of a prediction step, in which a new belief over the state is calculated based on the action $a_{T-1}$ taken, followed by an update step, which incorporates the likelihood of the observation $o_{T}$ to generate a new posterior. At each time step $T$, the robot takes an action and receives an 
observation. The prediction step incorporates the most recent action:

$$
P\left(s_{T} \mid a_{0: T-1}, o_{1: T-1}\right)=\int_{s_{T-1}} P\left(s_{T} \mid s_{T-1}, a_{T-1}\right) P\left(s_{T-1} \mid a_{0: T-2}, o_{1: T-1}\right) d s_{T-1} .
$$

In the above equation, we informally use the integral over the state even though, in general, the state may consist of continuous and discrete dimensions. Incorporating the new observation yields the posterior belief at time $T$ :

$$
P\left(s_{T} \mid a_{0: T-1}, o_{1: T}\right)=\frac{P\left(o_{T} \mid s_{T}\right) P\left(s_{T} \mid a_{0: T-1}, o_{1: T-1}\right)}{P\left(o_{T} \mid o_{1: T-1}\right)}
$$

The denominator is simply a normalizer to ensure the posterior belief integrates to one and in many practical cases, is not computed.

In the following chapters, we discuss the application of recursive Bayesian filtering in various forms to the problem of mechanism identification and estimation. We explore the details of filtering in this domain and show successful results with this approach in simulation and on a robot.

\subsection{Rationale}

Throughout this thesis, we will discuss our choices in not only our approach to the kinematic identification problem but also in the implementation of that approach. Our rationale for these choices stems from a desire to develop an extensible and generalizable approach.

\subsubsection{Choices}

- We design our system such that no human action is required during any identification experiments. Specifically, we assume the robot begins with its gripper attached to the mechanism's handle, which is accomplished through human intervention. The human is not involved after this point until the robot returns an estimate. 
- We choose to use "black-box" models of our mechanisms, as opposed to analytic models. We assume these models must meet four requirements.

- Given a previous state and an action, the model must return a new nominal state.

- Given a state, the model must return a noisy version of a state, where noise is only added to the state's configuration variables.

- Given a state, the model must return a nominal observation.

- The model must return a valid sample state.

- We use simple displacements as our actions. These actions or more complex actions can be used as input to our "black-box" models. Given our other assumptions, if the model can use a specified action to return a nominal state, the rest of our approach can remain unchanged.

- We use position sensing of the robot's end effector as our observations. We explore the performance of this type of sensing for the mechanism identification problem. However, different and potentially multiple sensors could be used as long as $P(O \mid s)$ can be calculated. As long as the question, "How probable is this observation returned from this sensor given a possible state of the world?" can be answered, the type and number of sensors used will not change the rest of our approach.

- We use uniform prior belief distributions. However, the prior distribution is arbitrary. In fact, the prior distribution is a natural component of the system in which to incorporate information about the environment such as contextual information about the objects in a room. However, we do not rely on strong priors for the performance of our approach.

- We use simple, approximate models of the mechanisms. Our simulation models are, by necessity, approximate. We do not model dynamics or higher order effects such as friction or jamming. We also model the mechanism alone without 
incorporating the robot's kinematics into the system. More precise models can readily be added thanks to "black-box" modeling. However, we demonstrate our system's performance using approximate models.

- In Chapter 3, we use three different action-selection strategies. Other actionselection techniques can be used without affecting the rest of our estimation approach.

\subsubsection{Requirements for a New Problem}

These choices are motivated by our desire for our approach to be applicable to other domains and problems. Various parts of our strategy must be specified for a new problem.

- The action and observation domains must be specified.

- Given the choice of actions and observations, transition and observation models must be supplied.

- The prior belief must be given and may incorporate any information desired.

- A method of selecting actions must be specified.

- The real world or a simulated version of it, of course, is required.

\subsection{Contributions}

This thesis makes four contributions:

1. We develop robust techniques for a robot to identify and estimate novel mechanisms.

2. We formulate the mechanism identification problem as a Bayes filter and present a simple discrete filter to demonstrate the potential of this choice in the domain (Chapter 3). 
3. We consider two important aspects of our hybrid belief space. First, we discuss the appropriateness of continuous state-space, parametric filters in the domain. Second, we describe three important consequences of Bayesian filtering in a hybrid space for hypothesis comparison and how they will influence the results of the inference (Chapter 4).

4. We apply a particle filter to the kinematic identification problem that drastically increases the size of the state space that can be considered. We demonstrate the effectiveness of this particle filter in simulation and on a real robot (Chapter 5).

\subsection{Thesis Organization}

In Chapter 2, we give an overview of work related to mechanism identification, estimation, and articulation using different techniques and different sensor modalities. In Chapter 3, we present the discrete Bayesian filtering approach to the mechanism identification problem. We show successful results in simulation on 6 different mechanism types and show significant improvements in speed of proper identification with active action-selection techniques. We discuss tests on the real robot with this filter. We also discuss the limitations of the discrete filter and the need for other techniques. In Chapter 4, we discuss the use of continuous state-space, parametric filtering for our problem. We conclude that parametric filters are inappropriate for this problem. The specific requirements of the problem which invalidate the use of these parametric filters and the complexity issues involved in the discrete filter point to using a particle filter which we introduce in Chapter 5 . We show the significant improvement that the particle filter brings in terms of its ability to tackle high dimensional state spaces. We demonstrate the effectiveness of this particle filter in simulation and on the robot. Finally, we discuss the limitations of the particle filter in this domain. 


\section{Chapter 2}

\section{Related Work}

There has been substantial previous work on kinematic identification and related problems.

\subsection{Vision-based Techniques}

Katz et al. [8] showed accurate identification of basic kinematic joint types (e.g. revolute, prismatic, but not latch) using vision-based tracking of features on a mechanism as it is actuated. The motion is created by a robot interacting with the mechanism by performing pre-defined actions. After tracking the motion of features on the object, feature clusters are formed based on their relative motion. The relative motion between clusters indicates the type of joint connecting the links of the mechanism. They consider revolute and prismatic joints between each cluster by providing models of the transforms between features on separate bodies. Improving on the performance of this previous work, Pillai et al. [16] use visual marker-less tracking to track the motions, created by human demonstration, of parts of a mechanism. They estimate the kinematic models constructed from different joint types from the visual data and demonstrate improved performance.

Similarly, Martín Martín and Brock [13] use visual RGB-D data to estimate the kinematic relationship between rigid bodies. Their approach uses a three-level filter, one to track image features, one to estimate rigid body motion, and one to estimate 
joint types between rigid bodies. They consider prismatic and revolute joints, rigid connections, and disconnections between rigid bodies. They show successful experimental results of their algorithm on real objects.

Brookshire and Teller [3] use visual data to estimate the pose of an articulated object. They use a completely specified kinematic model of the object and a specialized, dual particle filter to estimate the pose of the links from a series of observations. Their filter implementation allows for very poor transition models and can still accurately estimate the pose of the object even with noisy and intermittent observations.

Stürm et al. [21] also developed a sophisticated approach to kinematic identification from multiple information sources. They calculate a maximum-likelihood estimate of the parameters and probabilities over models for the joint. This work was restricted to four models for joints: rigid, revolute, prismatic, and Gaussian process, which is a data-driven model for any joint that could not be explained by the other three. The Bayesian Information Criterion was used to select the best model, trading off goodness of fit with model complexity. Three types of observations were used in the work. Much of the work used fiducial markers for tracking the individual links of the kinematic bodies. By analyzing the relative motion of the markers (much like Katz et al.), they were able to correctly identify joint types. They also provide some preliminary work on vision-based, marker-less tracking in some of their experiments. All of their vision experiments rely on a human moving the parts of the mechanism and all parts of the mechanism being visible.

Vision-based observations differ significantly from our gripper-position observations in the amount of information and possible applicable techniques to estimate mechanisms. The previously-discussed, vision-based systems must make the assumption of being able to track each part of the mechanism. In various ways, each of these works fits the parameters of various models to a set of observations and rates which model is most likely. These works do not focus on the robot's action selection to produce information from the world. In general, the body of work relies on information streams from object motion produced by scripted actions or humans, and, thus, their techniques do not reason about actions. 
Although these techniques are useful for many mechanisms, they do not extend to all mechanisms considered in this thesis. Specifically, the sequential latching mechanisms behave differently in different parts of their configuration space. Moreover, in some applications, not all parts of the mechanism are readily visible, which would prevent the use of these methods. Reasoning about the actions taken from various states is crucial to determine the behavior of these mechanisms. How to fit models to a set of observations from these mechanisms is unclear.

\subsection{Gripper-based Measurements}

Three closely related papers involve using gripper positions and forces for mechanism identification. In conjunction with Jain and Kemp, Stürm et al. [20] integrated gripper positioning while operating a mechanism. They use the controller Equilibrium Point Control (EPC) to actuate some simple mechanisms. A new equilibrium point at each step is calculated to keep a manipulator hook attached to the handle of the mechanism assuming the high-probability joint type. The equilibrium point then becomes the next commanded position. The position information is used to update the probabilities over joint types. Their system allowed a robot to successfully operate multiple mechanism types. Their approach does not use the action to help predict the joint type but instead relies on the data measured as actions are taken. The use of this controller relies on the initial action taken by the robot to provide informative motion of the parts of the mechanism. Jain and Kemp [5] use EPC again to estimate the kinematics of mechanisms, which are assumed to be either revolute or prismatic joints, lying in a plane parallel to the floor. This approach is employed successfully in other works (Rühr et al. [18] and Becker et al. [2]).

In later work, Jain and Kemp [6] show a data-driven approach to identifying specific doors, identifying door types, and detecting manipulation events such as locked doors or collisions between doors and other objects. By collecting training data, including forces and positions, while opening various types of doors, their system can correctly identify information of interest when encountering a new door instance. 
The first two works provide strategies for autonomous action selection, as does our work. However, these controllers rely on generating enough motion initially to provide a good estimate of the joint. They must be provided with useful initial actions or start with prior information about the mechanism. Our work demonstrates action selection techniques that can identify mechanisms even after no initial motion. The third work is concerned with identifying new door instances from previous data but does not address how to generate the learning or testing data autonomously without useful initial actions or prior information about the mechanism.

\subsection{Action Selection}

Katz et al. [9] use action selection methods based on relational reinforcement learning. They show that using this learning-based, action-selection method can significantly reduce the number of actions required to correctly identify the kinematic relationships in the structure. Their results demonstrate joint identification using guided learningbased action selection.

Narayanan and Likhachev [14] develop an approach for simultaneous identification and actuation of a mechanism to complete a task. Choosing appropriate actions to achieve this goal makes this setting a planning problem. To make solving the planning problem tractable, they assume, first, that all parts of any mechanism are observable without noise and, second, that the transition model is deterministic. They use a visual pipeline to provide candidate mechanisms to initialize the planner. The number of candidate mechanisms bounds the number of successor states from a belief state. Their representation can model mechanisms where the joint type between parts is a function of their poses. Their system was able to successful achieve a goal position when actuating a office cabinet and drawer.

Our active action-selection techniques consider the future belief and attempt to distinguish between mechanism hypotheses. We do not assume that we can measure the position of each part of a mechanism as this assumption is likely difficult to fulfill in real experiments with arbitrary mechanisms. We also do not have any system to 
narrow the possible hypotheses before our robot interacts with the mechanism. Our approach aims to identify the mechanism and its parameters such that the robot may properly actuate the mechanism as part of a future high level task. We do not assume that when encountering an unknown mechanism, the robot will have a clear actuation goal.

\subsection{Environment Exploration}

A number of works address the problem of exploring the environment to gain information about the degrees of freedom and kinematic structure of the objects. This field is related to the subproblem of mechanism identification.

Höfer et al. [4] attempt to learn kinematic background knowledge instead of kinematic models explicitly. They assume that their perception can segment rigid bodies and give their poses. Their goal is to learn, in the least number of actions, a transition model from experiences. They reward the robot for discovering new degrees of freedom. They assume that they can identify the joint type from the rigid body motions.

Otte et al. [15] provide techniques for exploring the degrees of freedom of an environment. They look at a more global problem of choosing objects in the world to explore with a single "push" action with the aim of discovering what degrees of freedom exist between objects. They use the articulation library developed by Stürm et al. [21] to estimate the joint types between rigid bodies. Otte et al. also infer other parameters of the joints such as friction and joint limits. Also, they offer strategies to address the possible trans-dimensionality of the belief space. They use entropy-based techniques to choose an object with which to interact. They show the effectiveness of their approach in simulation.

Our work relates to that of Höfer et al. and Otte et al. in the subproblem of mechanism identification. We do not address where to explore an environment to find mechanisms. Given that Otte et al. use Stürm et al.'s articulation library, the mechanism identification subproblem in their work differs from ours in the same as 
as Stürm's differs from ours. Otte et al. do not choose between actions but instead choose between objects on which they perform a single action with previously supplied knowledge of the objects. Moreover, they assume their world is fully observable. Höfer et al. also assume they known the poses of the rigid bodies in their environment. These full-observability assumptions may be hard to fulfill when applying their work to real robot experiments.

Kulick et al. [11] address the related but different problem of discovering the joint-dependency structure between joints connecting rigid bodies. For example, the configuration of one joint may lock another. They introduce a probabilistic model of these structures. Also, they use active action selection using a cross-entropy metric and change-point detection. They show the effectiveness of their approach for estimating joint dependency in simulation and on a PR2 robot. Their problem differs from the one in this thesis as they assume the robot has fully-parameterized, kinematic models of the entire environment. Therefore, they are not trying to discover what joints exist but instead how they depend on each other.

\subsection{Our Approach}

Our work differs significantly from this earlier work. We use a Bayes filter to identify the type of the mechanism without segmenting out or identifying the individual joints. Also, our fundamental approach does not require visual information. Our strategy can use any combination of information sources but is demonstrated in this paper using position information. In our work, we expand the range of possible mechanisms that can be identified and manipulated with only gripper-position feedback. In particular, we also consider "serial," or "sequential," mechanisms, such as latches, that behave differently in different parts of their configuration spaces. As implied in the name, these latches have a fixed constraint that their handles can be placed in to restrict their motion. We use "black-box" models of our mechanisms instead of analytic models used in many of the previous works. We also actively choose actions to gather

information quickly about the type of mechanism instead of directly attempting to 
control the mechanism as if it were of the most likely type. This exploratory action selection also allows the system to start with no information about how to move, even initially, and to discover appropriate motions through trial and error. 


\section{Chapter 3}

\section{Discrete Filter}

An alternative strategy is to represent a continuous conditional distribution of the parameters and variables for each mechanism type. We discuss this strategy and its difficulties in Chapter 4. First, we use a discrete representation of the state space, which makes the belief state a multinomial distribution. The mechanism types are discrete, and each mechanism type has its own set of parameters and variables, the spaces of which are uniformly discretized, so that a "state" in the discrete space corresponds to a multi-dimensional "box" in parameter-variable space. We let $\mathcal{S}$ be the set of discrete states

$$
s=\left\langle m,\left(\theta_{l o}, \theta_{h i}\right),\left(x_{l o}, x_{h i}\right)\right\rangle \in \mathcal{S}
$$

and let

$$
\hat{s}=\left\langle m,\left(\theta_{h i}-\theta_{l o}\right) / 2,\left(x_{h i}-x_{l o}\right) / 2\right\rangle
$$

be a canonical state value, with the values for parameters and variables chosen in the centers of their ranges. 


\subsection{Discrete Bayesian Inference}

Given the discrete state-space representation, we can easily discretize the continuous Bayesian inference formulation shown in Section 1.1.2 as

$$
\begin{aligned}
\operatorname{Pr}\left(m_{T}, \theta_{T} \mid a_{0: T-1}, o_{1: T}\right) & =\sum_{x_{T}} \operatorname{Pr}\left(m_{T}, \theta_{T}, x_{T} \mid a_{0: T-1}, o_{1: T}\right) \\
& =\sum_{x_{T}} \operatorname{Pr}\left(s_{T} \mid a_{0: T-1}, o_{1: T}\right) .
\end{aligned}
$$

Again, further marginalizing this expression over the states of the mechanism from times 0 through $T-1$ gives

$$
\sum_{x_{T}} \sum_{s_{0: T-1}} \operatorname{Pr}\left(s_{0: T} \mid a_{0: T-1}, o_{1: T}\right)
$$

Finally, as before, we can write

$$
\operatorname{Pr}\left(m_{T}, \theta_{T} \mid a_{0: T-1}, o_{1: T}\right) \propto \sum_{x_{T}} \sum_{s_{0: T-1}} \operatorname{Pr}\left(s_{0}\right) \prod_{t=0}^{T-1} \operatorname{Pr}\left(o_{t+1} \mid s_{t+1}, a_{t}\right) \operatorname{Pr}\left(s_{t+1} \mid s_{t}, a_{t}\right)
$$

The discrete version of the recursive filtering equations given in Section 1.1.3 are as follows. The prediction step is given by

$$
\operatorname{Pr}\left(s_{T} \mid a_{0: T-1}, o_{1: T-1}\right)=\sum_{s_{T-1}} \operatorname{Pr}\left(s_{T} \mid s_{T-1}, a_{T-1}\right) \operatorname{Pr}\left(s_{T-1} \mid a_{0: T-2}, o_{1: T-1}\right) .
$$

The update step is given by

$$
\operatorname{Pr}\left(s_{T} \mid a_{0: T-1}, o_{1: T}\right)=\frac{\operatorname{Pr}\left(o_{T} \mid s_{T}\right) \operatorname{Pr}\left(s_{T} \mid a_{0: T-1}, o_{1: T-1}\right)}{\operatorname{Pr}\left(o_{T} \mid o_{1: T-1}\right)}
$$

Just as before, at each time step $T$, the robot takes an action, receives an observation, and utilizes both to update its belief over the state space. 


\begin{tabular}{lll}
\hline$m$ & $\theta$ & $x$ \\
\hline Free & & $x, y$ location \\
Fixed & $x_{\text {pos }}, y_{\text {pos }}$ location & \\
Revolute & $x_{\text {pivot }}, y_{\text {pivot }}$ location, $r$ radius & $\alpha \in[0,2 \pi]$ angle \\
Prismatic & $x_{\text {axis }}, y_{\text {axis }}, \alpha_{\text {axis }}$ axis definition & $\beta$ displacement \\
Latch 1 & $x_{\text {pivot }}, y_{\text {pivot }}, r, \alpha_{\text {latch }}, \beta_{\text {latch }}$ & $\alpha, \beta$ \\
Latch 2 & $x_{\text {axis }}, y_{\text {axis }}, \alpha_{\text {axis }}, \beta_{\text {latch }}^{1}, \beta_{\text {latch }}^{2}$ & $\beta^{1}, \beta^{2}$ \\
\hline
\end{tabular}

Table 3.1: Space of possible mechanism states

\subsection{Experimental Domain and Implementation}

We have applied this general approach to the example problem of discriminating among six different types of mechanisms using position feedback resulting from manipulation of each mechanism at a designated handle. The mechanisms all operate in a plane, although this is not a fundamental limitation of the approach. Extensive experimental results were generated using a simulated robot. Additional experiments using a Willow Garage PR2 robot have shown successful diagnosis of instances of several mechanism types.

\subsubsection{States}

The mechanism types include a completely free body, a rigidly fixed body, a 1-DOF revolute joint, a 1-DOF prismatic joint, and two sequential 2-DOF latch mechanisms. The first latch is a composite mechanism comprised of a revolute joint followed by a prismatic joint. However, as implied by the name, the latch is more than simply a composite mechanism because a fixed rigid body exists in which the end of the prismatic joint can be placed. This constraint causes this model to exhibit different behaviors in different parts of its configuration space. Similarly, the second latch is comprised of a prismatic joint followed by another prismatic joint perpendicular to the first. This mechanism also has a fixed rigid body to restrict its motion in certain parts of the mechanism's configuration space. Furthermore, the prismatic joints in these models have displacement limits along their axes.

Table 3.1 shows the types, parameters, and variables that make up the state space. 
Figure 3-1 shows the definition of each model, its parameters, and its variables. The variables are written in blue while the parameters are written in red. The rotated " $U$ " shaped boxes around the handles (represented by the large dot in each diagram) of the latch mechanisms remain fixed in space even while the mechanism moves to different configurations. For the prismatic mechanism, the dashed line representing the axis is meant to indicate that the handle can move freely along this axis. This free motion along the axis of the prismatic model is distinctly different from the constrained motion that the prismatic joints exhibit in the two latch models. A dashed axis line is absent from these diagrams to indicate the existence of these limits.

The free model can move freely in the plane and has configuration variables $x$ and $y$ and no parameters. Conversely, the fixed model cannot move and, thus, has no configuration variables but instead has parameters $x_{p o s}$ and $y_{\text {pos }}$ referring to the model's fixed position in the plane. The revolute model is parameterized by its radius $r$ and its pivot position given by $x_{\text {pivot }}$ and $y_{\text {pivot }}$. The configuration of the revolute model is its angle from the x-axis $\alpha$. The prismatic model's parameters are an axis point given by $x_{a x i s}$ and $y_{a x i s}$ and an axis angle $\alpha_{a x i s}$. The displacement from the axis point $\beta$ is the model's configuration. The latch mechanisms have parameters which specify the position of the constraint as well as parameters and configuration variables for the movable part of the mechanism. For Latch 1, the parameters of the movable portion are its radius $r$ and pivot point given by $x_{\text {pivot }}$ and $y_{\text {pivot }}$. This portion's configuration is the angle of the revolute joint $\alpha$ and the displacement of the prismatic joint $\beta$. The constraint is parameterized by it's angle $\alpha_{\text {latch }}$ and $\beta$, a maximum displacement of the prismatic joint while $\alpha=\alpha_{\text {latch }}$. For Latch 2 , the parameters of the movable part are the angle of the axis $\alpha_{a x i s}$ and the pivot point of the axis given by $x_{a x i s}$ and $y_{a x i s}$. The movable portion's configuration variables are $\beta^{1}$ and $\beta^{2}$, the displacements of the prismatic joints along the first and second prismatic axes respectively. Finally, the parameters of the constraint are $\beta_{\text {latch }}^{1}$ and $\beta_{\text {latch }}^{2}$, the displacements along the first and second prismatic axes respectively that lead to the position of the constraint. 


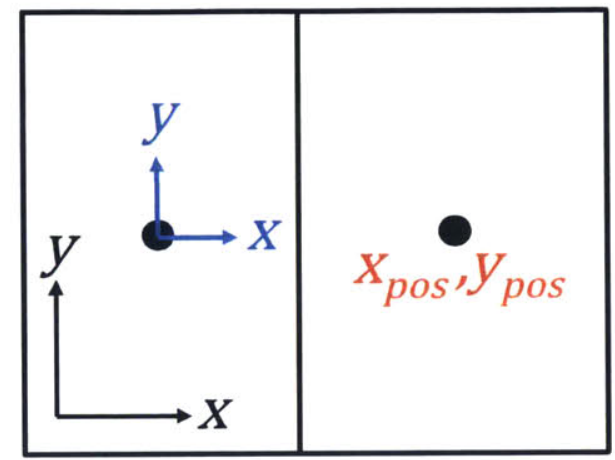

Free Fixed
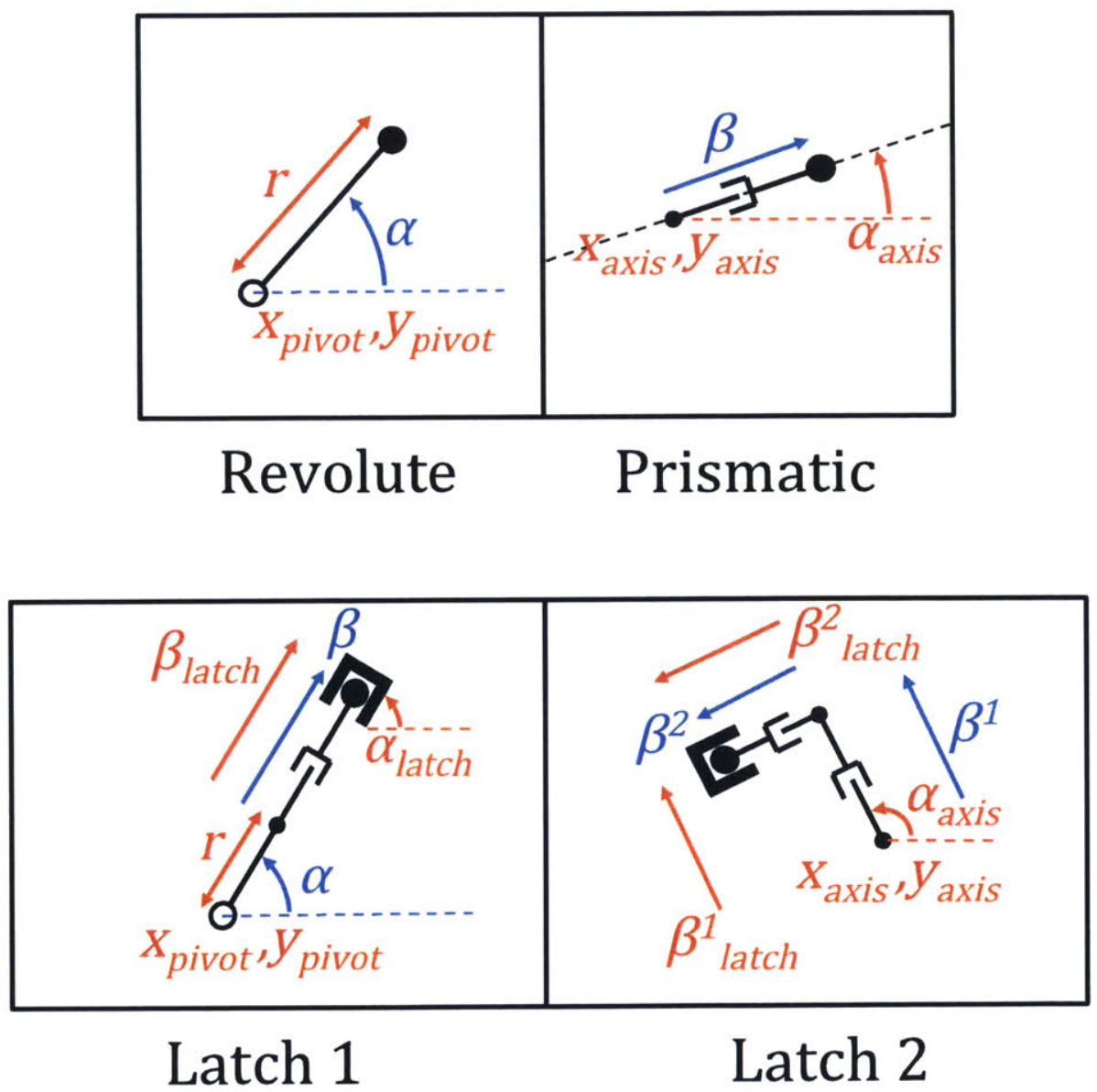

Figure 3-1: Diagrams of the each of the six models considered. Fixed parameters are shown in red while variables are shown in blue. The large dot represents each mechanism's handle. 


\subsubsection{Actions}

In this work, we restrict our attention to physical interactions in which we assume the robot is grasping the mechanism without rotational coupling. That is the robot is rigidly attached to the handle in translation put applies no torque to the mechanism through the handle. We assume that the robot will work within a finite workspace. Furthermore, we assume that the robot, while moving the handle of a particular mechanism, will have no probability of generating a state in which the handle is placed outside of the workspace (e.g., the robot will not throw a free body out of its reach). Actions consist of commands to move the hand to target poses in the horizontal plane. The commands are executed by a proportional-derivative (PD) controller for a fixed amount of time. The gains of the controller are chosen to drive the gripper close to the target position, and the time-out is such that, if no obstacles are encountered, then any target point in the robot's workspace can be reached. However, the robot may not reach the target point if the mechanism in its current state constrains the motion.

On the real robot, we choose the gains of the controller such that the robot exhibits a high stiffness in the direction it attempts to move but a low stiffness perpendicular to that direction. The overall level of these gains is low to avoid damaging the robot or the mechanism. This type of compliant motion increases the size of the movements observed when actuating the real mechanisms, which have friction and can jam. We have verified in our pilot tests with the PR2 that the compliance in the robot controller and the mechanisms are such that incomplete motions do not generate large enough forces to damage the mechanisms. When the action terminates, the gripper is commanded to its current position with the effect of "relaxing" the robot. We assume that the real robot can perform the command displacements and does not perform unintended motions such as moving its gripper out of the plane. However, these unintended motions cannot be eliminated completely.

The actions can be specified as absolute or relative. The absolute actions allowed for the robot are a discrete set of target points within its workspace. The robot's 
workspace is constrained to a $30[\mathrm{~cm}]$ by $30[\mathrm{~cm}]$ square in the horizontal plane centered at the grippers start pose. In the experiments discussed in Section 3.5, the target points are nine poses on an axis-aligned, regularly-spaced, $12[\mathrm{~cm}]$ grid as shown in Figure 3-2(a). Starting from any pose in the workspace, the robot attempts to move to one of these points.

Relative actions are Cartesian displacements from the gripper's current pose. With any action-selection technique, the robot never chooses an action that has a target pose outside of the workspace. Used in the experiments discussed in Section 3.6.3, the relative actions are eight equally spaced $12[\mathrm{~cm}]$ motions radially away from the gripper as shown in Figure 3-2(b).

\subsubsection{Observations}

In this domain, we use the robot's proprioception, in the form of the observed $(x, y)$ position of the object's handle (the same position as the robot's end effector) as the observation. The relationship between the commanded position and the resulting position yields information about the underlying mechanism.

\subsubsection{Transition and Observation Models}

For many idealized situations, writing down transition and observation models analytically is possible by using ideal physics models to describe the nominal next state and observation and adding Gaussian noise. However, once collisions and friction are involved, generating analytical models becomes much more difficult. Our approach in this work is to use the Bullet Physics Library (http://www. bulletphysics.org) to construct approximate models of the mechanisms, and then use those models to simulate the effects of actions and to model the observations. The use of simulation allows significant flexibility in terms of the possible object classes that can be modeled.

A consequence of using a simulation rather than an analytical model is that we do not have the ability to integrate effectively over states and observations; in our 


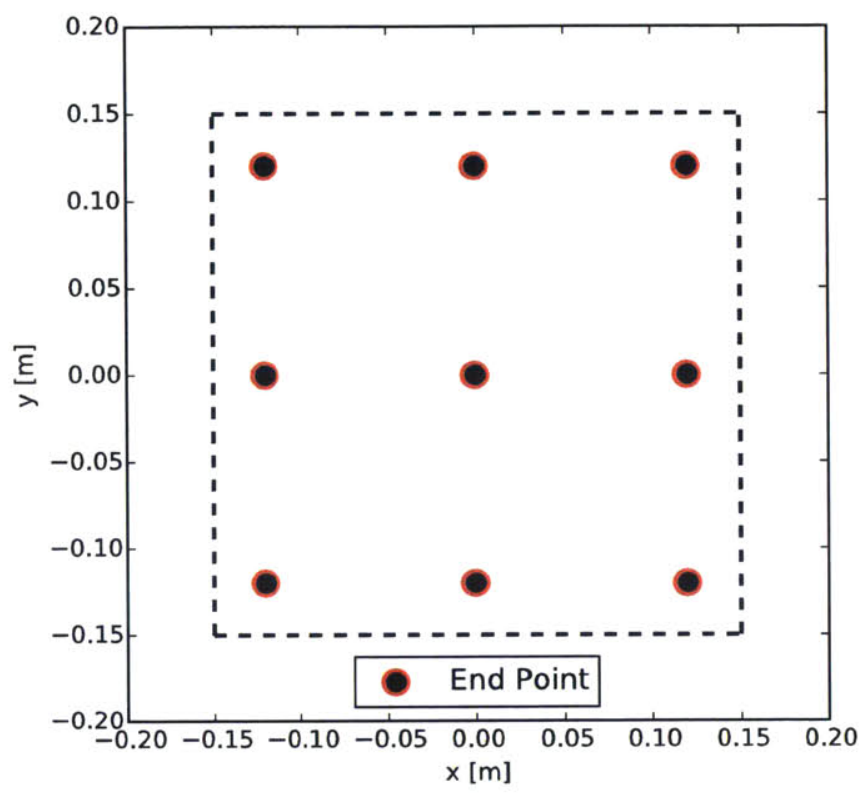

(a) Absolute Actions

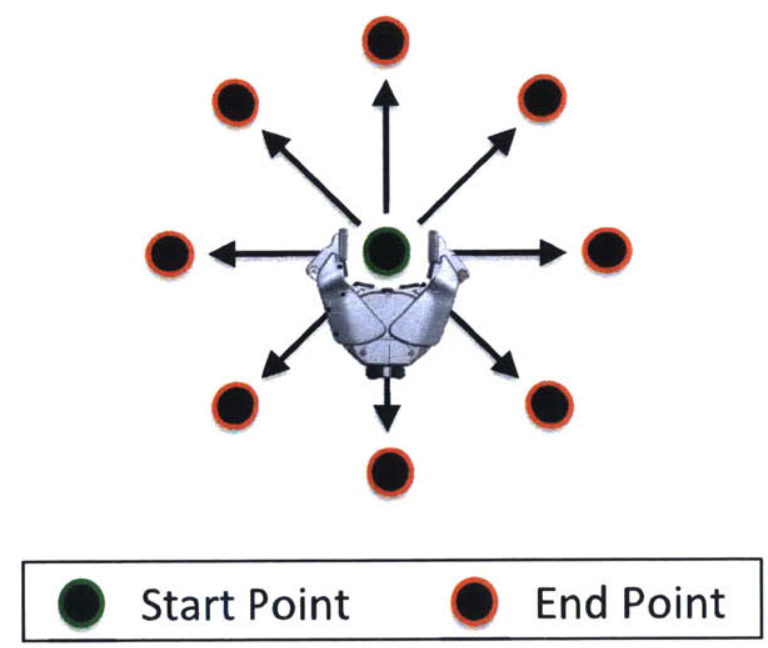

(b) Relative Actions

Figure 3-2: a) The robot's absolute actions are attempted moves from any point in the workspace to one of the designated end points. The workspace is denoted as the dashed, black line. The end target points are shown as black dots with red outlines. Absolute actions are used in the experiments discussed in Section 3.5. b) The robot's relative actions are attempted moves from the gripper's current pose in one of eight directions. The start pose is denoted as a black dot with green outline. The end target points are shown as black dots with red outlines. Relative actions are used in the experiments discussed in Section 3.6.3. 
implementation we have to rely on samples drawn from the simulation.

The transition and observation models used in the estimator have Gaussian noise around nominal next states and observations. This simple choice is a gross approximation of the truth but is sufficient in this context. In order to compensate for possible modeling errors and to better demonstrate the effects of the action-selection strategies, we use a significantly higher variance in the observation model than would be expected in the real robot.

Specifically, we calculate transition and observation probabilities using Gaussian distributions in the observation space. To calculate $\operatorname{Pr}\left(s_{T} \mid s_{T-1}, a_{T-1}\right)$, we simulate the state $s_{T-1}$ forward with action $a_{T-1}$ which produces a nominal next state $\hat{s}_{T}$. We map $\hat{s}_{T}$ to $\hat{o}_{T}$ in observation space. Then, to calculate the probability of each $s_{T}$, we map it to $o_{T}$ and evaluate the Gaussian with mean $\hat{o}_{T}$ and a diagonal covariance matrix of variances $\sigma_{\text {trans }}^{2}$ at $o_{T}$. Similarly, to calculate $\operatorname{Pr}\left(o_{T} \mid s_{T}\right)$, we map each state $s_{T}$ to $o_{T}$ and evaluate the Gaussian with mean $o_{T}$ and variance $\sigma_{o b s}^{2}$ at $o$, the true observation. We choose the variances for all dimensions of the transition and observation covariance matrices to be $\sigma_{\text {trans }}=0.01[\mathrm{~m}]$ and $\sigma_{o b s}=0.10[\mathrm{~m}]$ respectively.

\subsubsection{Simulated Robot and "True" Mechanism}

We simulate the robot's interactions with the mechanism. Not attempting to model the PR2's complexity, we model the robot simply as a point which can move the mechanisms handle. At each time step, a simulation separate from the filter is used to calculate the mechanisms motion. The simulation calculates a nominal position in observation space, and the same artificial noise is added to all dimensions. This noise is meant to mimic the combined approximate transition and observation noise of a real system and is set to $\sigma_{\text {true }}=0.01[\mathrm{~m}]$. The level of noise is below the chosen values for the filter discussed in Section 3.2.4 above. Note that we write "true" in quotation marks as a reminder that the mechanism does not physically exist. 


\begin{tabular}{ll}
\hline$m$ & $\theta$ \\
\hline Free & \\
Fixed & $0.0,0.0$ \\
Revolute & $0.3,0.3,0.3 \sqrt{2}$ \\
Prismatic & $-0.16,-0.16, \pi / 4$ \\
Latch 1 & $-0.2,0.0,0.1,0.0,0.1$ \\
Latch 2 & $-0.1,-0.1,0.0,0.1,0.1$ \\
\hline
\end{tabular}

Table 3.2: Experimental parameter values for mechanism types

\subsubsection{Experimental Details}

For the following experiments, we restrict our state space to one parameter set for each of the 6 model types considered. However, adding another set of parameters for a model type is no different than adding a single parameter set for a new model type. Thus, the approach is capable of using a state space with many parameter sets for each model type, although the state-space size grows as the product of the number of parameter and variable values for each type. Each model-parameter pair has a discrete set of variable values which span the robot's workspace. The specific parameter values used for each model are given in Table 3.2.

When the world is initialized, the robot assumes with certainty that its gripper's current position is $(0,0)$ in the workspace. The robot then performs actions relative to this position. Therefore, for any model-parameter pair to be considered with nonzero probability, the pair must have the Cartesian position $(0,0)$ as an achievable pose relative to the robot's gripper. When simulating the "real" world, a "true" mechanism was chosen from among the possible mechanism types considered. Any observation from this "true" mechanism received added Gaussian noise. The initial variable values of each "true" mechanism were chosen such that the handle would begin at $(0,0)$ as required. Note that the latching mechanisms were initialized in their latched position.

\subsection{Active Exploration}

Random or arbitrary selection of actions is rarely efficient, in the sense that it may require a long sequence of actions to effectively determine the type and parameters of 
the mechanism the robot is interacting with. We can articulate the goal of identifying the mechanism as desiring that the conditional entropy,

$$
H\left(m_{T}, \theta_{T} \mid a_{0: T-1}, o_{1: T}\right)
$$

be below some desired value. The entropy of a random variable is a measure of its disorder or lack of information; in the discrete case, it is

$$
H(X)=-\sum_{x} \operatorname{Pr}(x) \log _{2} \operatorname{Pr}(x)
$$

We hope that the robot's actions and observations increase its certainty of the mechanism. A distribution with nearly all mass on one hypothesis suggests certainty of that hypothesis. This type of distribution also has low entropy. Thus, decreasing entropy is a natural metric on which to choose actions. We might have an additional implicit goal that the robot not change the type or parameters of the mechanism; this would prevent the robot from ripping the door off its hinges in order to reduce it to an easily identifiable free body.

Finding an optimal strategy for selecting observations to reduce overall entropy is as difficult as solving a POMDP. For efficiency reasons, we will pursue a myopic action-selection strategy. Given a belief state $b$, which is a probability distribution over the state of the mechanism at time $T$ given all previous actions and observations, the objective is to select the action that, in expectation over observations it may generate, will result in the belief state $b^{\prime}$ with the lowest entropy. That is:

$$
a_{T}^{*}=\min _{a} E_{o \mid b, a} H\left(s_{T+1} \mid a_{0: T-1}, o_{1: T}, a, o\right)
$$

Depending on the sizes of the spaces, this can be difficult to calculate analytically; we describe a sampling method in Section 3.2.

In problems that are submodular, this greedy strategy can be shown to be within a constant factor of optimal [10]. For a problem to be submodular, it must be that taking a particular action $a_{1}$ is never more informative after taking another action 
$a_{2}$ than it would have been before taking $a_{2}$. Unfortunately, that is not the case in our domain: attempting to rotate a latch may only be informative after it has been translated into a configuration in which the rotational freedom is available.

Even though it is not even boundedly suboptimal, we will find empirically that greedy action selection is a significant improvement over a random approach.

\subsection{Critical Computations}

The two critical computations in the system are the belief-state update and action selection.

The belief update takes a belief state from the previous time step, an action, and an observation, and computes an approximate new belief state.

$\operatorname{BELIEFUPDATE}(\mathbf{b}, \mathrm{a}, \mathrm{o})$ :

$1 \quad b^{\prime}=\operatorname{ZEROS}(b)$

2 for $s \in \mathcal{S}$

$3 r=\operatorname{SimTRANS}(\hat{s}, a)$

$4 \quad$ for $s^{\prime} \in \mathcal{S}$

$5 \quad b^{\prime}\left[s^{\prime}\right]=b^{\prime}\left[s^{\prime}\right]+\mathcal{N}\left(\hat{s}^{\prime}, r, \sigma_{\text {trans }}\right)$

6 for $s \in \mathcal{S}$

$7 \quad z=\operatorname{SimOBS}(\hat{s}, a)$

$8 \quad b^{\prime}[s]=b^{\prime}[s] \cdot \mathcal{N}\left(z, o, \sigma_{o b s}\right)$

9 return NORMALIZE $\left(b^{\prime}\right)$

Lines 2-5 compute the transition update. Given a discrete state $s$, we find the canonical continuous state, $\hat{s}$, and then find the nominal next continuous state, $r$ by invoking the simulated transition model SIMTRANS. Now, we assign a transition probability from $s$ to each discrete $s^{\prime}$ in proportion to a Gaussian density with variance $\sigma_{o b s}^{2}$, evaluated on the distance between $r$ and $\hat{s}^{\prime}$, which is the canonical continuous value of state $s^{\prime}$. At the end of this update, $b^{\prime}$ is an unnormalized multinomial distribution over the discrete states, representing the effects of taking action $a$. Lines 6-8 compute the observation update. Given a canonical continuous state $\hat{s}$ and action $a$, we 
invoke the simulator SIMOBS to find the nominal observation $z$. Then, we scale the posterior belief in $s, b^{\prime}[s]$ by the Gaussian likelihood of making actual observation $o$ when expecting observation $z$, with variance $\sigma_{o b s}^{2}$. Finally, we divide through by the sum of the values in $b^{\prime}$ and return a multinomial distribution.

The action selection procedure uses sampling to estimate the expected entropy resulting from taking each possible action, and returns the minimizing action.

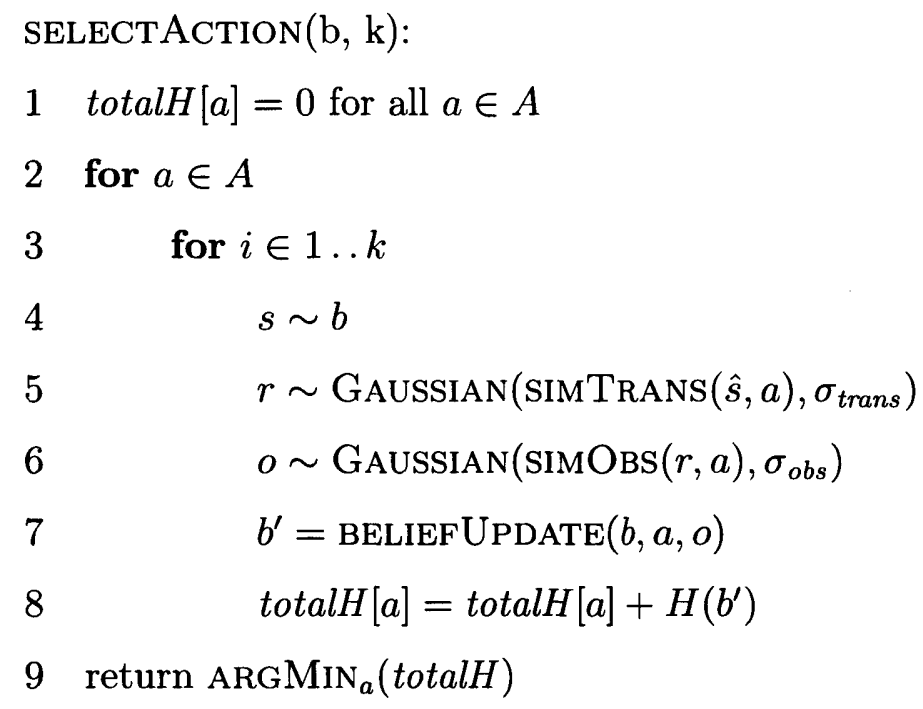

For each action, we draw $k$ sampled observations and compute the average entropy of the resulting belief states. Then we return the action that minimizes that average entropy. Lines 4-6 generate a sample observation by: sampling a discrete state $s$ from $b$, then sampling a continuous resulting state $r$ from a Gaussian centered on the nominal dynamics model applied to canonical continuous state $\hat{s}$ and action $a$, then sampling an observation $o$ from a Gaussian centered on the nominal observation for continuous state $r$.

\subsection{Results}

Experimental results were obtained in simulation. Each of the 6 mechanism types was used as the true model. For a given experiment or trial on a given mechanism, the robot took 10 actions, and the filter's belief state over the mechanism and parameters 


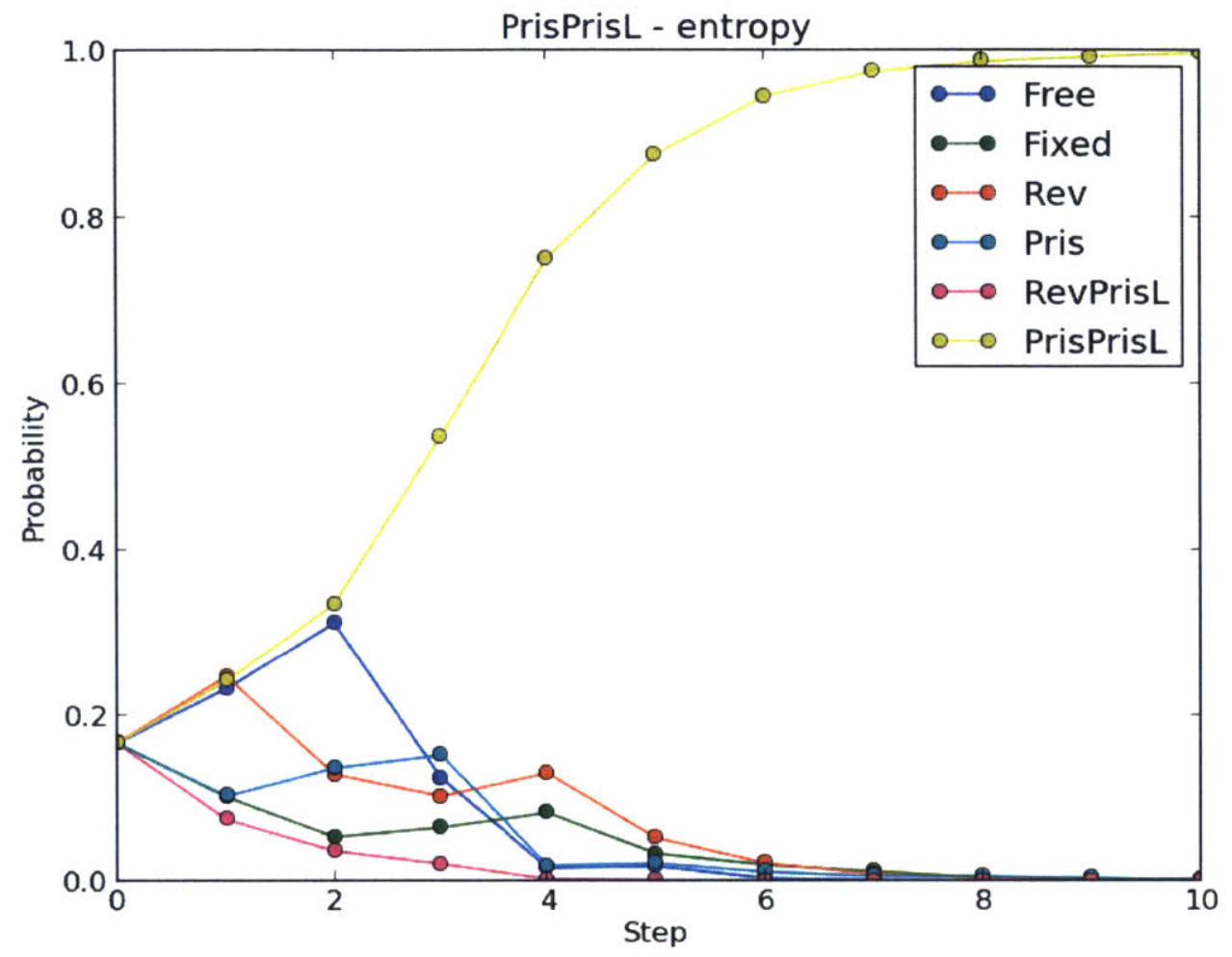

Figure 3-3: Single trial for instance of the Latch 2 model type using entropy-based action selection.

was recorded (after summing over all possible variable values for a given modelparameter pair). Each trial was repeated 10 times, and the results were averaged.

\subsubsection{Single Trial}

Considering a single trial on a particular mechanism can clearly illustrate the process of entropy-based action selection. Figure 3-3 shows the results from a trial with the true mechanism of type Latch 2 with the parameters given in Table 3.2 for that mechanism. A model instance is a particular pair of model type and parameter set which completely define the behavior of the mechanism. The first three actions chosen during this trial were $(0.12,-0.12),(0.12,0.12)$, and $(-0.12,0.12)$. These values are given in meters in the horizontal, $(x, y)$ Cartesian plane.

The first action caused increased belief in the Latch 2 model instance (the same 
type and parameters as the true mechanism), the revolute model instance, and the free model instance. These three models are the only models which could come close to the position achieved by the true mechanism. Concretely, the true mechanism can almost reach the commanded position because the handle can slide out of the latching constraint and move within a short distance of the commanded point. The instances of the free and revolute models can also come near this position. However, the fixed model instance cannot move. The prismatic model instance moves along an axis that does not come near to the commanded point. Finally, the Latch 1 model instance is restricted by its latching constraint and also cannot move.

The second action chosen further increases the belief that the true mechanism is either the free or Latch 2 model instances. However, the revolute instance's probability decreases sharply because the commanded move takes the true mechanism's position within the radius of the revolute instance.

The third action distinguishes between the last two instances that have high probabilities. Although the true mechanism can exist in a configuration state near the commanded target point, the mechanism begins in a position such that the action causes it to collide with the fixed latching constraint rigid body. If the true mechanism was the free instance, the handle should move completely to the commanded point. Because the robot cannot reach the commanded point, the observation obtained drastically decreases the belief in the free instance leaving only the Latch 2 instance with high probability. The following actions further solidify the belief in the Latch 2 instance, and thus, the estimator correctly identifies the true mechanism.

This example shows an important result. Considering the previous configuration of the mechanism, the system can choose an action for high information gain that may not have been a useful action in other possible configurations of the mechanisms considered. This behavior illustrates the usefulness of action selection based on the entropy of the belief state. By choosing actions carefully, the system is able to distinguish the true model from the other possibilities with relative few actions compared to random exploration. 


\subsubsection{Full Trials}

Figure 3-4 and Figure 3-5 show two main results. The left column of each figure shows the averaged experimental results for random action selection while the right column shows the results for entropy-based action selection. In nearly all cases (for each mechanism and each type of action selection), the true model-parameter set became much more likely than the other possibilities after less than 10 actions and, in most cases, after only a few. Moreover, the speed of convergence is significantly improved by the entropy-based action selection. In these experiments, entropy-based action selection requires between half and two-thirds of the number of actions required by random action selection to reach the same confidence level.

Using physics simulations, the filter was able to robustly predict the two different latch mechanisms. These mechanisms can experience collisions with the fixed part of the latch. Second, the latches themselves are built with limits to make them realistic models of latches found in the real world. For example, Latch 1 can only be extended to a certain distance even outside of the locking constraint. These limits are a type of unilateral constraint which can be hard to model analytically. However, the use of simulations can more easily model many complex real world systems.

In all but 1 of 120 trials, the filtered classified the mechanism correctly. In the trial that failed, actions were selected randomly. In this experiment, the true Latch 1 model was misclassified as the fixed mechanism after 10 actions because no selected action moved the handle out of the latch's constraint. After 10 actions, the models had nearly identical probability, but the fixed mechanism was slightly favored due to discretization error. This result emphasizes the benefit of entropy-based action selection as it would have purposefully chosen actions to disambiguate between the two models in this scenario.

These simulation results do not test variations in the parameter values of the model types. Many different instances of each model type could be added to the state space allowing the filter to decide within a model type which parameter set is more likely. However, the state-space size scales as the product of the number of 


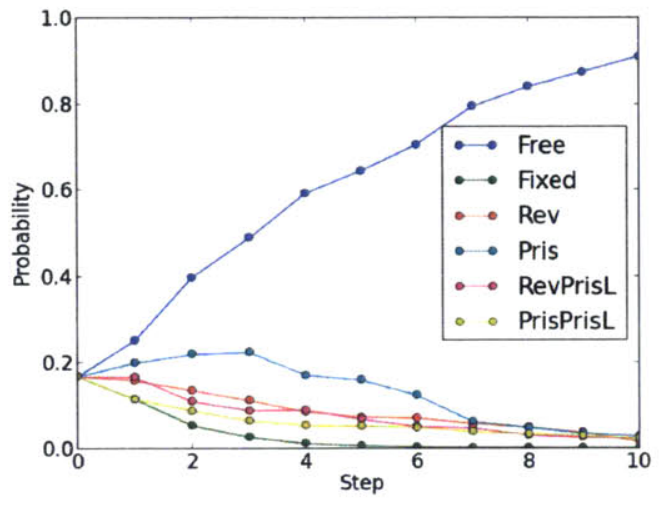

(a) Free - Random

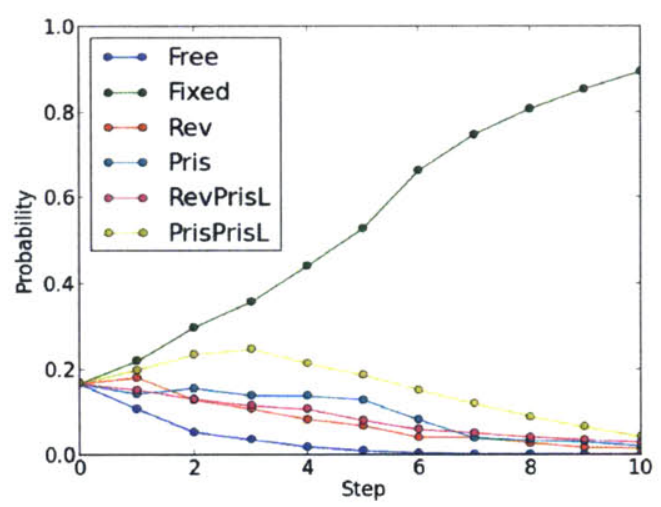

(c) Fixed - Random

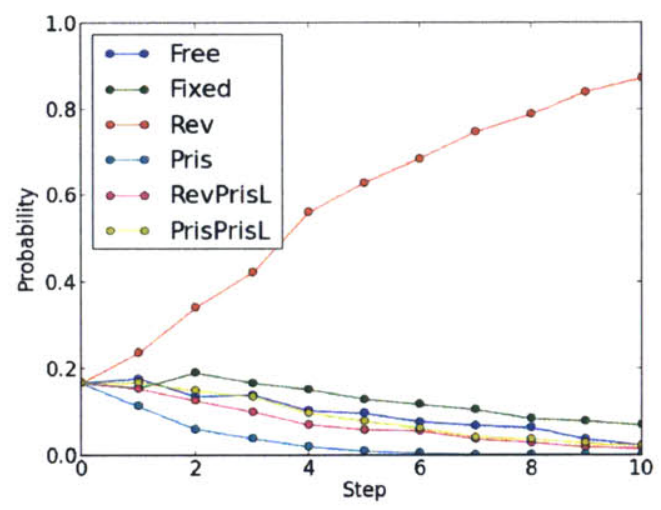

(e) Revolute - Random

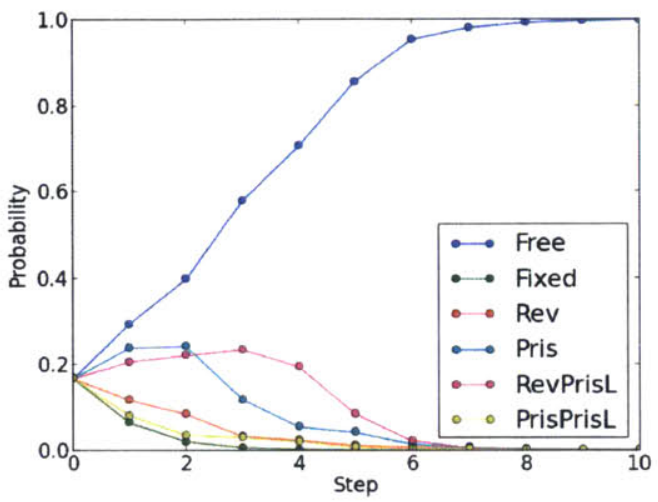

(b) Free - Entropy

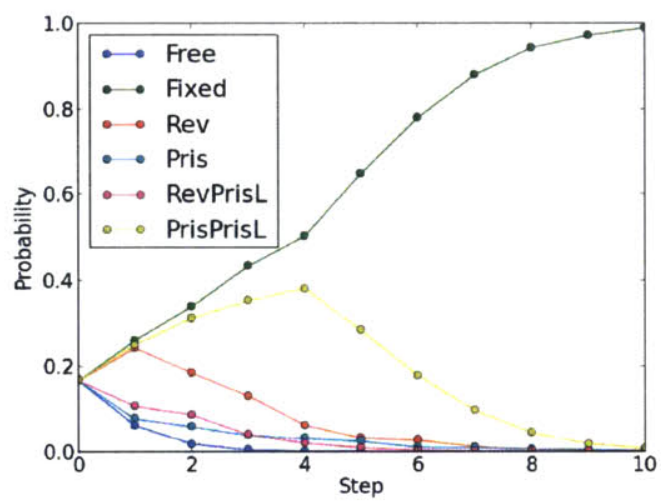

(d) Fixed - Entropy

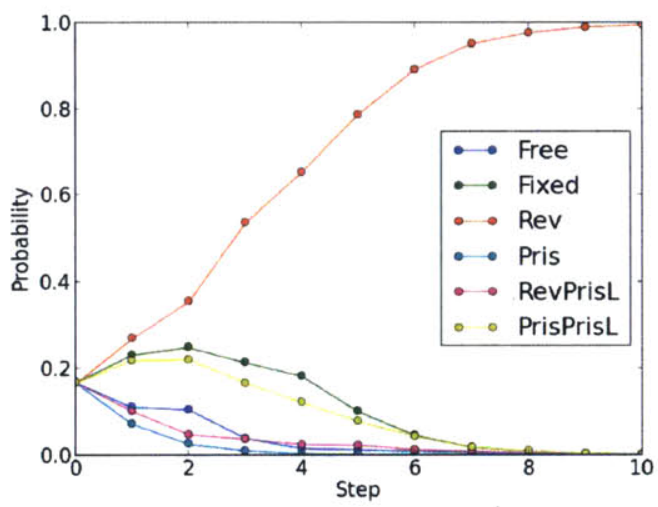

(f) Revolute - Entropy

Figure 3-4: Filter convergence and random vs. entropy-based action selection from free, fixed, and revolute models. 


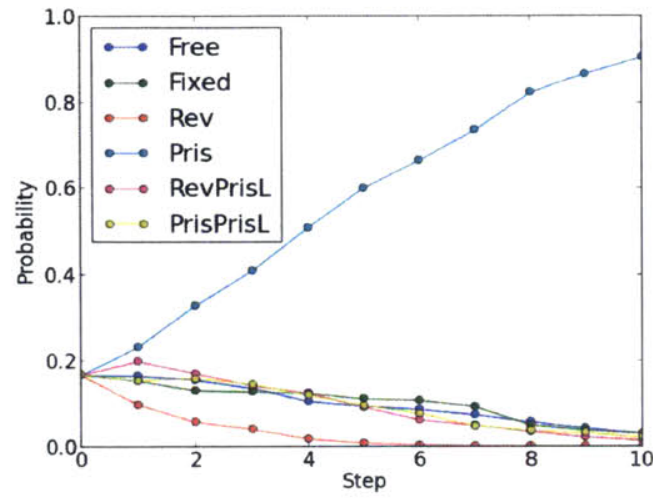

(a) Prismatic - Random

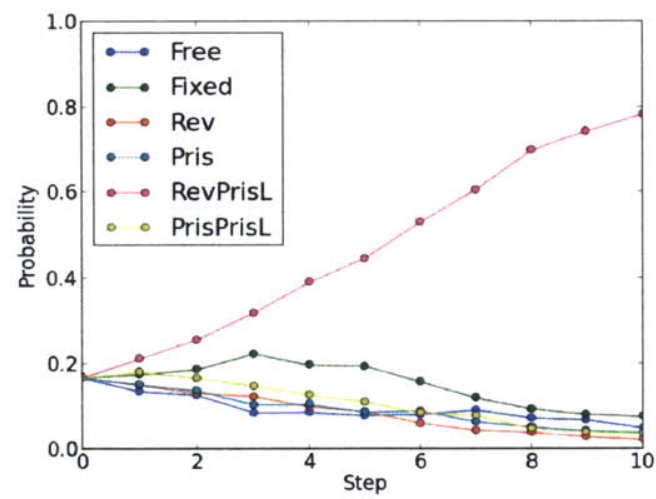

(c) Latch 1 - Random

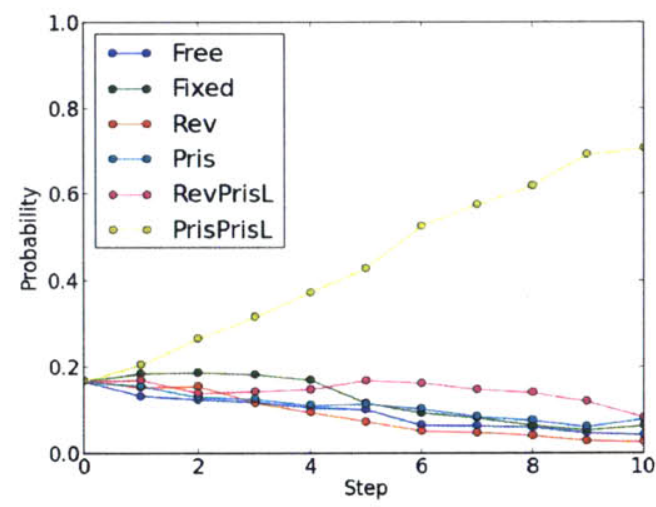

(e) Latch 2 - Random

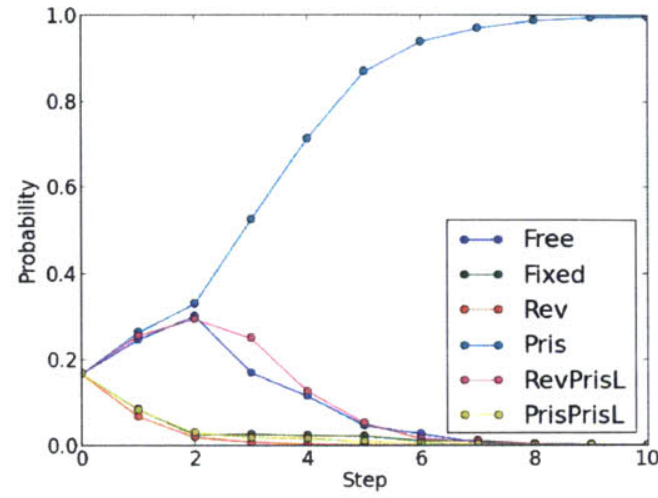

(b) Prismatic - Entropy

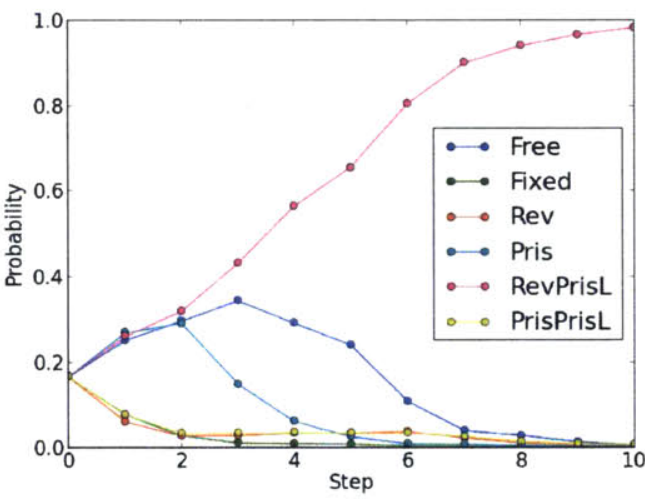

(d) Latch 1 - Entropy

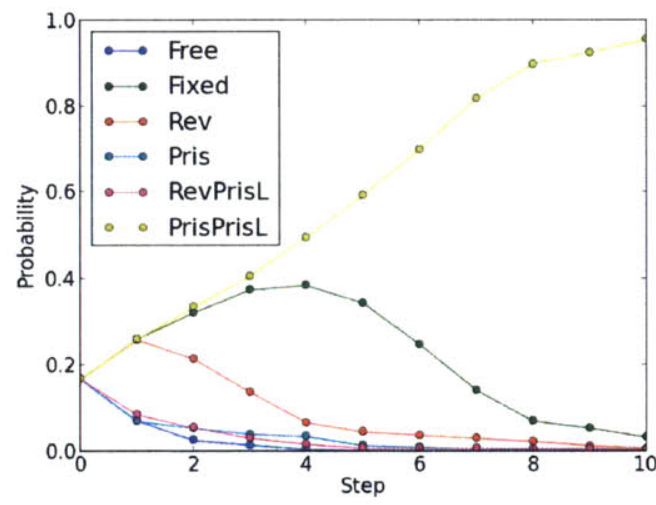

(f) Latch 2 - Entropy

Figure 3-5: Filter convergence and random vs. entropy-based action selection from prismatic, Latch 1, and Latch 2 models. 
variable and parameter values, summed over types. The filter update time grows as the square of the state-space size. Action selection requires a filter update for each sample of the belief state for each action considered. Thus, with a sufficiently large state space, this method becomes computationally intractable.

We conducted additional experiments on the Willow Garage PR2 robot. The results suggest that the robot is able to distinguish between instances of several of the models. The corresponding video (http://lis.csail.mit.edu/movies/ICRA14_ 1756_VI_fi.mp4) shows examples of these successful trials. While the real world experiments are very promising, they can fail due to factors that are not present in the simulations. For example, out-of-plane displacements can cause the mechanism to bind. Future implementations suffer from the same disparity between the real world and the transition model. Chapter 5 specifically addresses the problem of poor modeling.

\subsection{Expanding the Space}

We hope to expand the state space that our discrete filter can address. As mentioned above, our first round of simulation experiments do not test multiple parameter sets per mechanism type. With only one parameter set per type, type identification and parameter estimation are indistinguishable. In principle, we can easily add more parameter sets per type.

However, as mentioned above, the computational complexity of the discrete filter limits, in a practical sense, the size of the problem that can be solved. In this section, we test the practical limit of the discrete filter. First, we add more parameter sets per mechanism type. Second, we describe a new action-selection technique that aims to provide the performance boost of entropy-based action selection without the computational cost. Although the filter updates themselves are costly, the complexity of entropy-based action selection limits us to a much smaller state space than the discrete filter could handle using random action selection. We show that the new action-selection method is a significant improvement over our initial methods and 


\begin{tabular}{ll}
\hline$m$ & $\theta$ \\
\hline Free & \\
Fixed & $0.0,0.0$ \\
Revolute 1 & $0.44 / \sqrt{2}, 0.44 / \sqrt{2}, 0.44$ \\
Prismatic 1 & $0.16,0.16,-3 \pi / 4$ \\
Latch 1 1 & $0.27,0.0,0.17,-\pi, 0.1$ \\
Latch 2 1 & $-0.1,-0.1,0.0,0.1,0.1$ \\
Revolute 2 & $-0.44 / \sqrt{2},-0.44 / \sqrt{2}, 0.44$ \\
Prismatic 2 & $0.23,0.0,0.0$ \\
Latch 1 2 & $-0.27,0.0,0.17,0.0,0.1$ \\
Latch 2 2 & $0.1,0.1,-\pi, 0.1,0.1$ \\
\hline
\end{tabular}

Table 3.3: Experimental parameter values for mechanism types

discuss remaining obstacles to further scaling.

\subsubsection{Expanded State Space}

To expand the state space, we add to the original state space described in Section 3.2.6. The free mechanism does not have any parameters, and the fixed mechanism must always have the same parameters. Therefore, an additional parameter set for each of the revolute, prismatic, and two latch models grows the space to 10 model-parameter pairs total. Table 3.3 gives the parameter values used in simulation for the 10 model-parameter pairs. In the names for each model-parameter pair, we use the distinction " 1 " or " 2 " to indicate the 2 different parameter sets for a mechanism type. This nomenclature should not be confused with the distinction between the first and second latch which are the same as in Figure 3-1. The original, smaller space had 292 discrete states. The expanded space has 456 discrete states, an increase of approximately 56\%. Recall that the update step is $O\left(n^{2}\right)$ and thus would see a $144 \%$ time increase.

\subsubsection{Statistical Racing}

As described in Section 3.3, selecting actions randomly can be very inefficient in terms of the number of actions required to identify a mechanism. Myopic entropyminimizing action selection can significantly decrease the number of actions required 
to achieve the same confidence in the correct hypothesis. However, the time complexity of the action-selection algorithm completely overshadows the belief update. Thus, for certain size spaces and problem specifications, the increase in computational time may partially mitigate the time savings from needed fewer actions.

We aim to develop an approximate, heuristic action-selection strategy to reduce the number of required actions while avoiding large complexity increases. From intuition about the mechanism identification problem, we propose to use a form of statistical racing that closely resembles a horizon-1 version of the planing approach taken by Platt et al. [17]. The robot executes an action on the mechanism and receives an observation. The robot can predict the motion of any of its hypothesis mechanisms. Considering the nominal observation obtained from any of these motions from the various models, the robot should choose the action that causes the largest distance between nominal observations.

A large distance between nominal observations should help distinguish hypotheses in the filter. This result follows from a Gaussian observation model with finite variance. The robot receives one observation $o$ per action from the world. The observation likelihood scales inversely with the distance between $o$ and a nominal observation $\tilde{o}$. Consider two nominal observations $\tilde{o}_{1}$ and $\tilde{o}_{2}$ from different hypotheses. If the distance between $\tilde{o}_{1}$ and $\tilde{o}_{2}$ is small, the difference in their corresponding likelihoods will likely also be small. Conversely, if the distance between $\tilde{o}_{1}$ and $\tilde{o}_{2}$ is large, the difference in likelihoods will also be large in general. Thus, we can expect that choosing an action that leads to large distances between the resulting nominal observations will lead to large likelihood differences which will, in turn, allow us to distinguish between the hypotheses.

The racing action-selection technique finds two high-probability states at each time step and attempts to choose an action to distinguish between them. 


$$
\begin{aligned}
& \text { SELECTACTIONRACING }(\mathrm{b}, \mathrm{k}) \text { : } \\
& 1 \operatorname{dist} O[a]=0 \text { for all } a \in A \\
& 2 \quad b_{M P}=\int_{x} b(m, p, x) d x \\
& 3 m p_{\text {first }}=\operatorname{ARGMAX}_{m, p}^{\text {first }}\left(b_{M P}\right) \\
& 4 m p_{\text {second }}=\operatorname{ARGMAX}_{m, p}^{\text {second }}\left(b_{M P}\right) \\
& 5 s_{\text {first }}=\operatorname{ARGMAX}_{s}\left(b\left(m, p=m p_{f i r s t}, x\right)\right) \\
& 6 s_{\text {second }}=\operatorname{ARGMAX}_{s}\left(b\left(m, p=m p_{\text {second }}, x\right)\right) \\
& 7 \text { for } a \in A \\
& 8 r_{\text {first }} \sim \operatorname{GAUSSIAN}\left(\operatorname{SimTRANS}\left(\hat{s}_{\text {first }}, a\right), \sigma_{\text {trans }}\right) \\
& 9 \quad o_{f i r s t} \sim \operatorname{GAUSSIAN}\left(\operatorname{SIMOBS}\left(r_{f i r s t}, a\right), \sigma_{o b s}\right) \\
& 10 \quad r_{\text {second }} \sim \operatorname{GAUSSIAN}\left(\operatorname{SIMTRANS}\left(\hat{s}_{\text {second }}, a\right), \sigma_{\text {trans }}\right) \\
& 11 o_{\text {second }} \sim \operatorname{GAUSSIAN}\left(\operatorname{SIMOBS}\left(r_{\text {second }}, a\right), \sigma_{o b s}\right) \\
& 12 \operatorname{dist} O[a]=\operatorname{DiSTANCE}\left(o_{\text {first }}, o_{\text {second }}\right) \\
& 13 \text { return } \operatorname{ARGMAX}_{a}(\text { distO })
\end{aligned}
$$

Specifically, at each step, we inspect the distribution over model-parameter pairs, namely the belief marginalized over the configuration variables $x$. We select the model-parameter pairs with the two highest probabilities. Then, for each modelparameter pair, we choose the highest probability configuration. Each action in the list of valid actions, actions which do not move the handle out of the workspace, is executed starting from each of these two states. For each action, we record the distance between nominal observations and select the action which leads to the largest inter-observation distance.

By selecting actions to suppress one of a pair of hypotheses at each step, eventually, and reasonably efficiently, only one hypothesis or a group of similar hypotheses should remain. Note that although the robot chooses an action to disambiguate between only two hypotheses, that action can provide substantial information about the other hypotheses as well. As seen in the following experimental results shown in Section 3.6.3, this type of action selection quickly suppresses many hypotheses with each action. In the following section, we compare the performance of this heuristic technique alongside the random and entropy-minimizing action-selection methods. 


\subsubsection{Results}

Similarly to Section 3.5, we test, in simulation, the performance of the discrete filter on the larger state space. Each experiment or trial consists of the 10 actions chosen by either the random, entropy-minimizing, or statistical-racing action-selection techniques. Again, 10 different model-parameter pairs of 6 different mechanism types make up the larger space. For each model-parameter pair as the "true" mechanism, we run 10 trials for each action-selection strategy. With 10 trials for 3 action-selection techniques for 10 model-parameter pairs, we run 300 experiments total. Filtering occurs in the larger space over each trial, and as before, we record for comparison the marginal probability distribution over model-parameter pairs after marginalizing out the configurations. The hope is that the belief places the most mass on the mechanism type and parameters of the "true" mechanism.

Figures 3-6 through 3-10 show the time history over experiments of the average probability for each model-parameter pair. Each line in each graph shows the average probability of the corresponding model-parameter pair over the 10 trials run for a particular "true" model-parameter pair. As in the previous results, for all action selection types, the averages probabilities always converge to the correct model-parameter pair by the end of the trial. The entropy-minimizing and statistical racing action selection show performance improvements over random. For every model-parameter pair, the two active techniques converge to higher probabilities with fewer actions. Note that the three action-selection techniques perform approximately equally well on the fixed model as expected because any action on this mechanism will lead to similar observations and large percentage of the possible actions will help distinguish this mechanism from the others.

Over 300 trials, only seven errors, in which an incorrect model-parameter pair had higher confidence, occurred. These errors occur due to the coarse discretization causing poor transition and observation likelihood calculations. For example, the world can produce an observation consistent with two separate model-parameter pairs. However, if the observation is closer to the nominal observation of a state of 


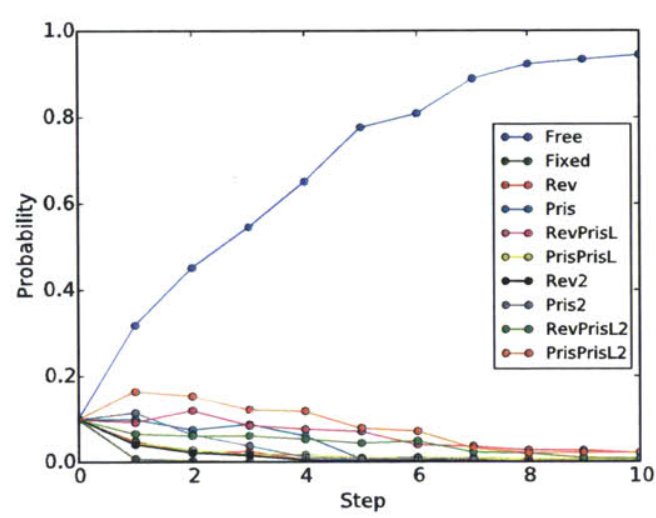

(a) Free - Random

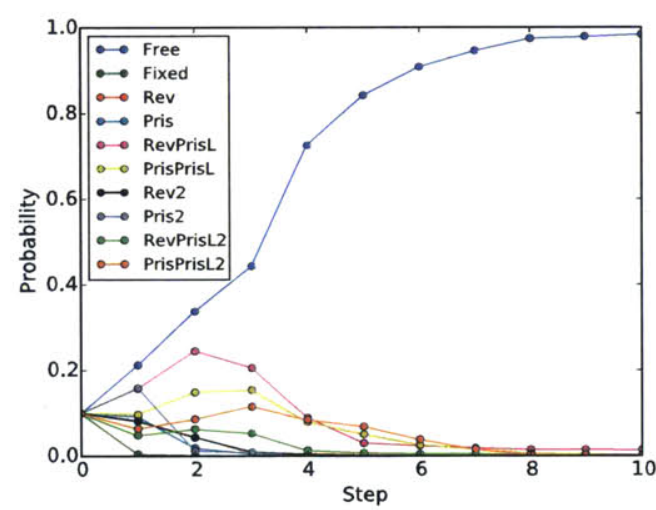

(c) Free - Entropy

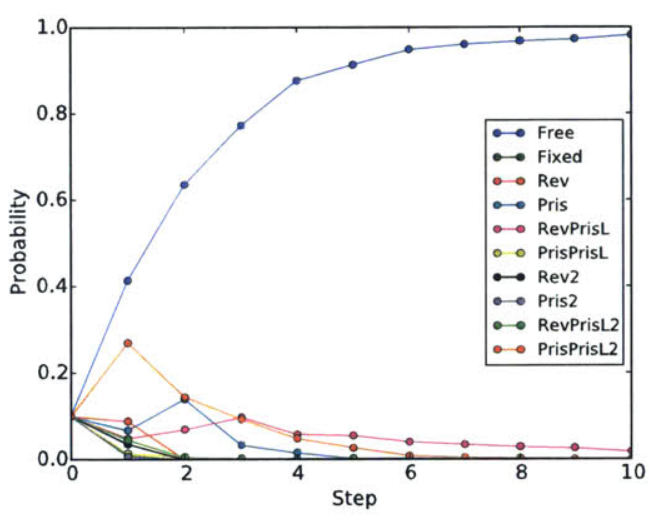

(e) Free - Racing

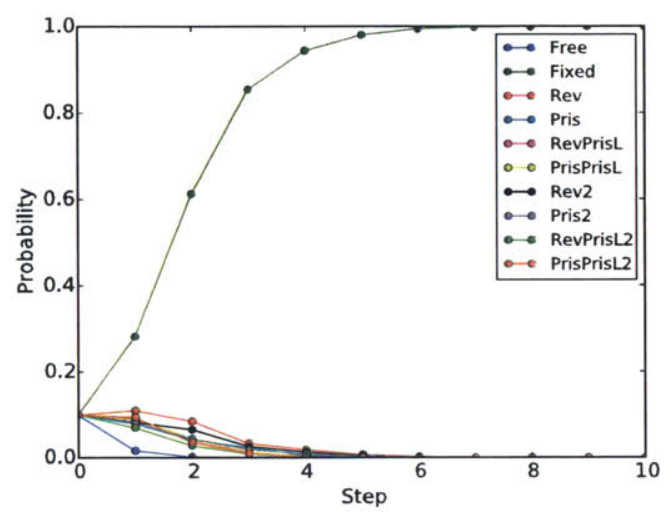

(b) Fixed - Random

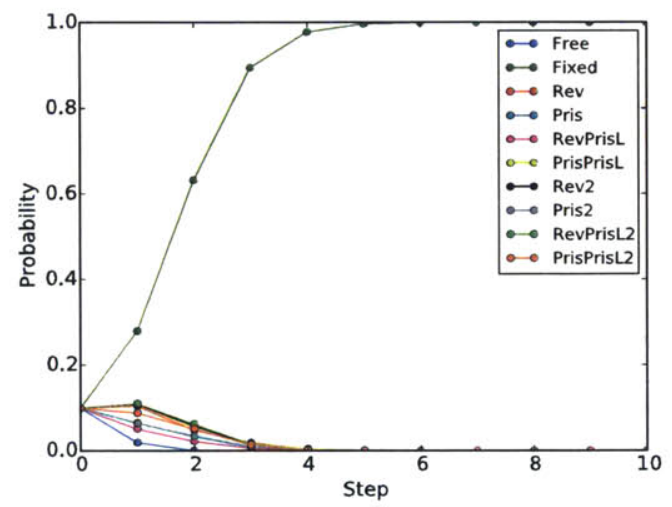

(d) Fixed - Entropy

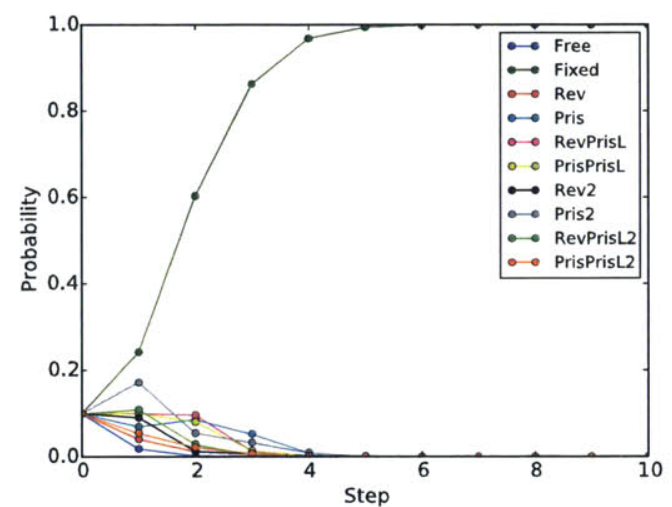

(f) Fixed - Racing

Figure 3-6: Filter convergence and random vs. entropy-based vs. statistical racing action selection from free and fixed models. 


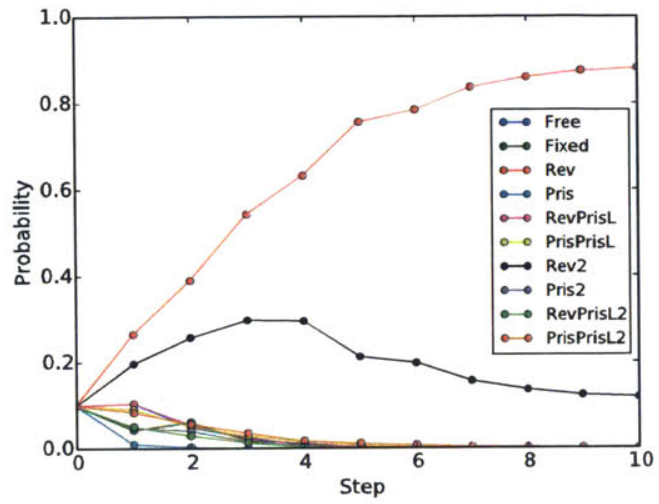

(a) Revolute 1 - Random

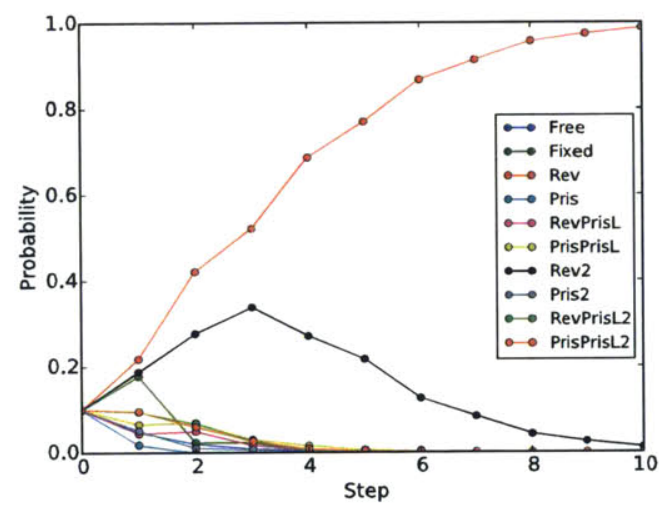

(c) Revolute 1 - Entropy

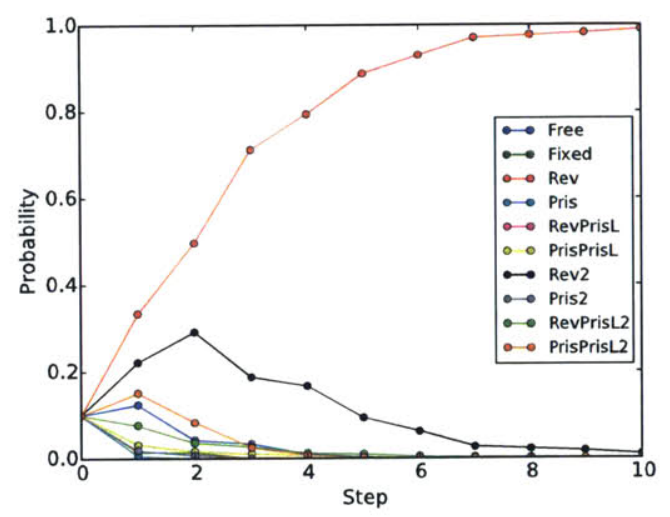

(e) Revolute 1 - Racing

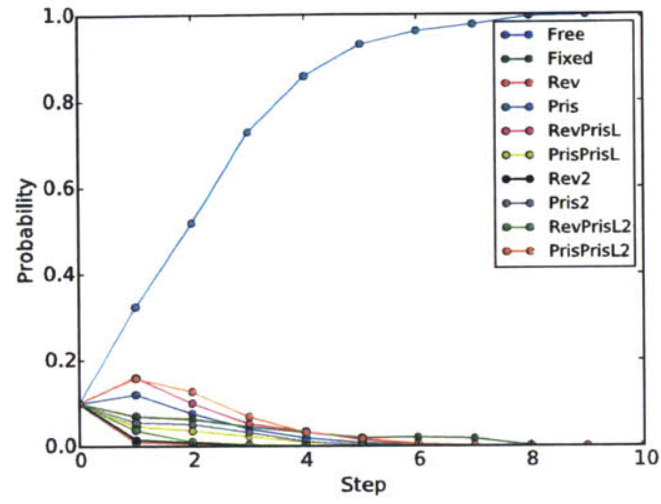

(b) Prismatic 1 - Random

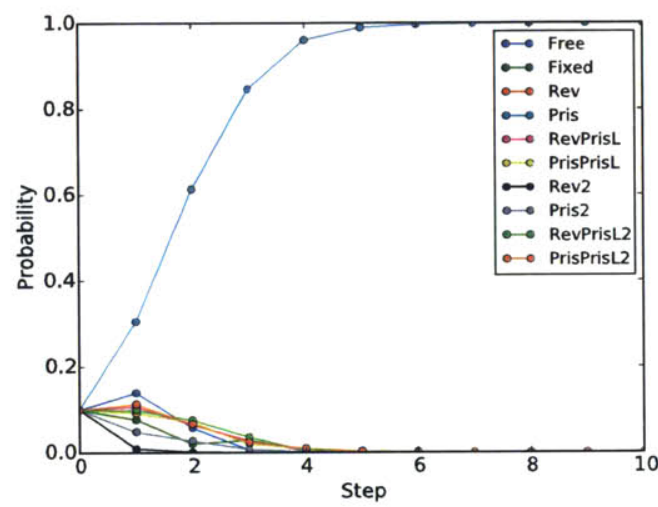

(d) Prismatic 1 - Entropy

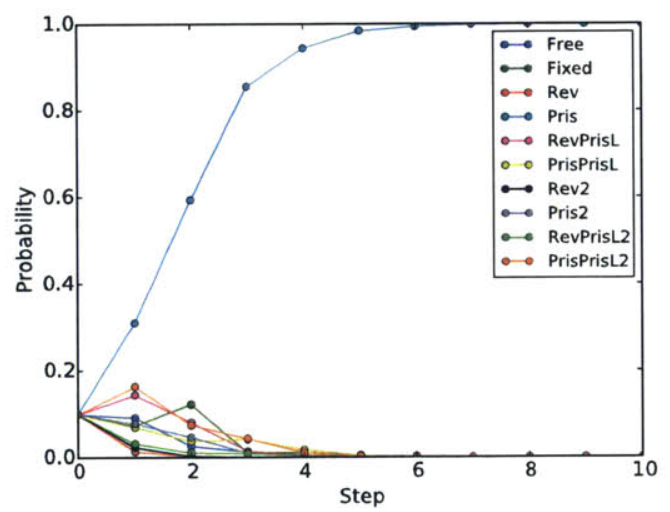

(f) Prismatic 1 - Racing

Figure 3-7: Filter convergence and random vs. entropy-based vs. statistical racing action selection from Revolute 1 and Prismatic 1 models. 


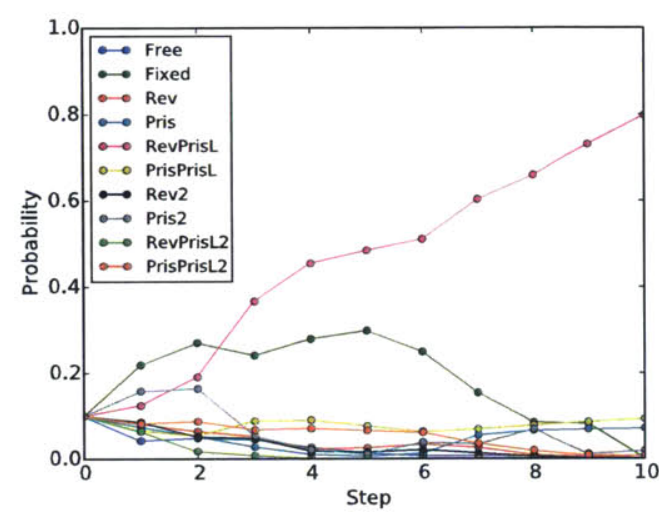

(a) Latch 11 - Random

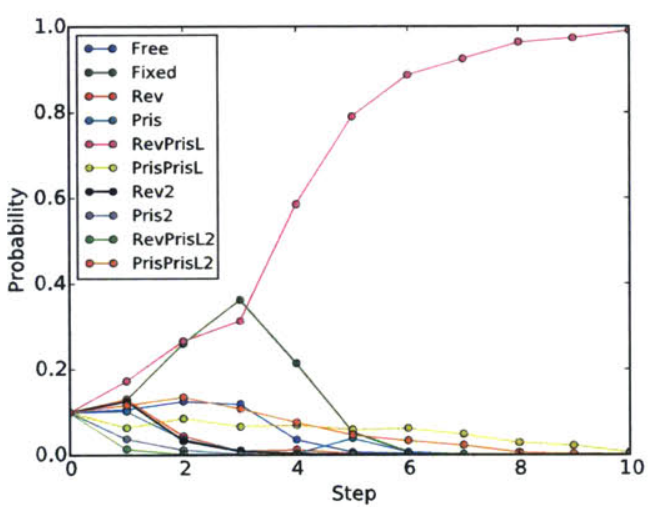

(c) Latch 11 - Entropy

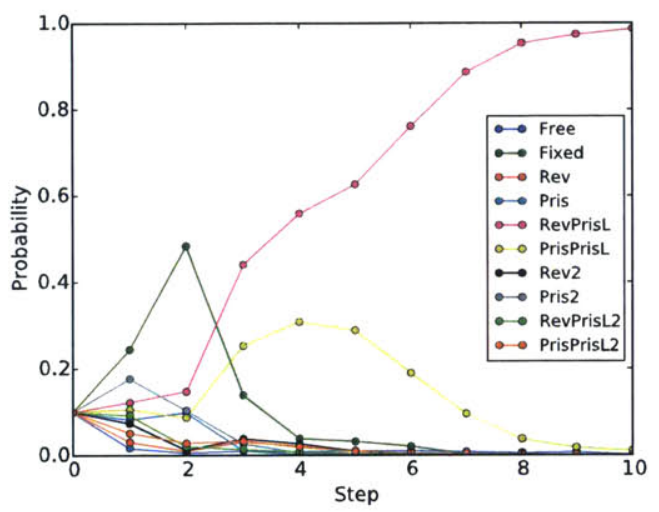

(e) Latch 11 - Racing

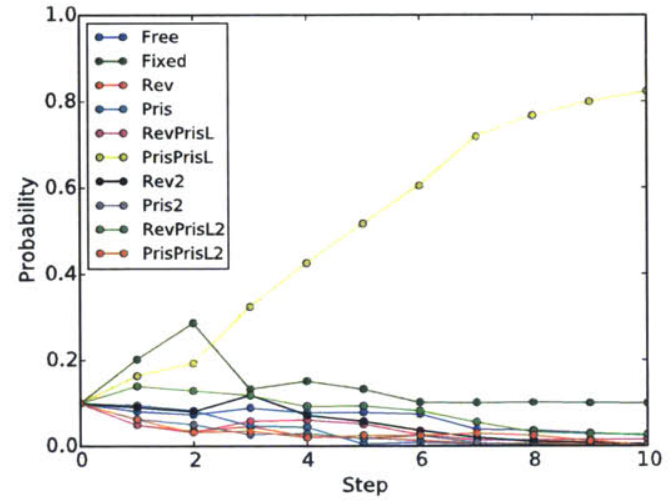

(b) Latch 21 - Random

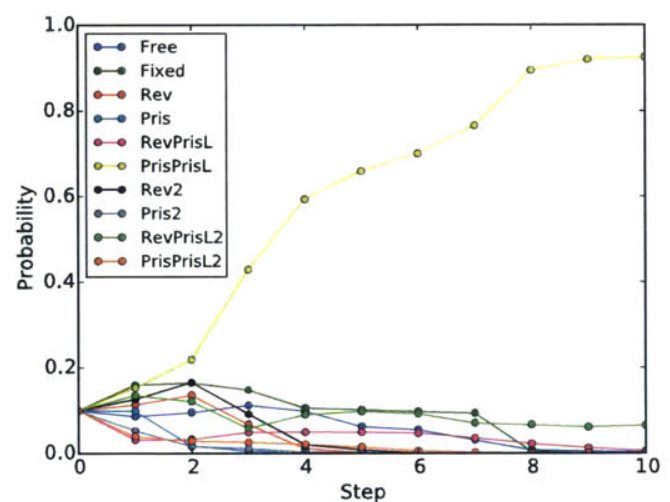

(d) Latch 21 - Entropy

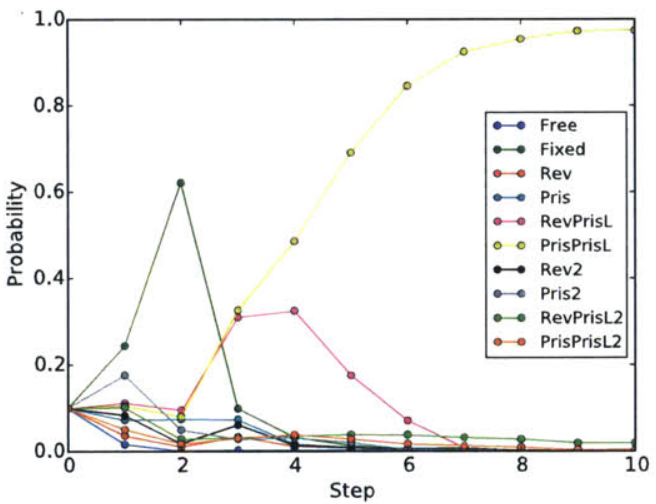

(f) Latch 21 - Racing

Figure 3-8: Filter convergence and random vs. entropy-based vs. statistical racing action selection from Latch 11 and Latch 21 models. 


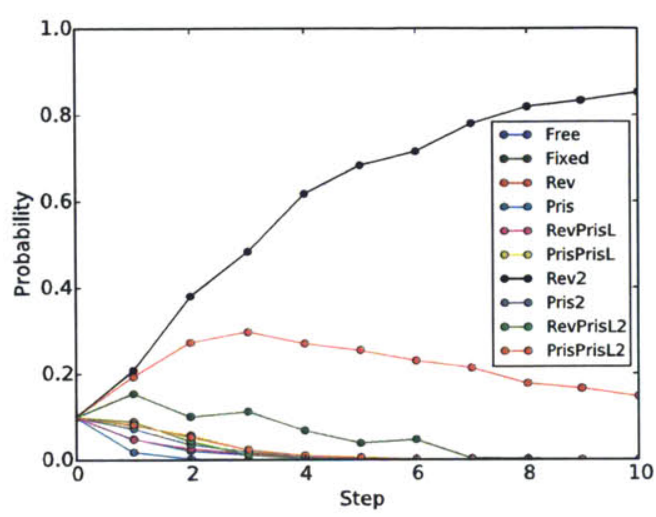

(a) Revolute 2 - Random

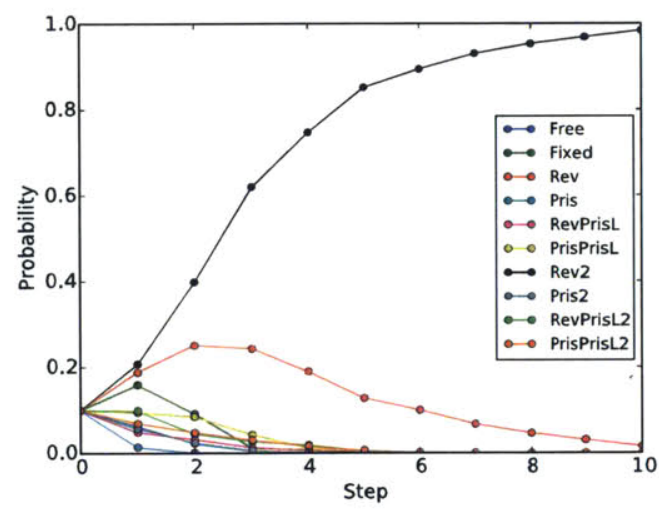

(c) Revolute 2 - Entropy

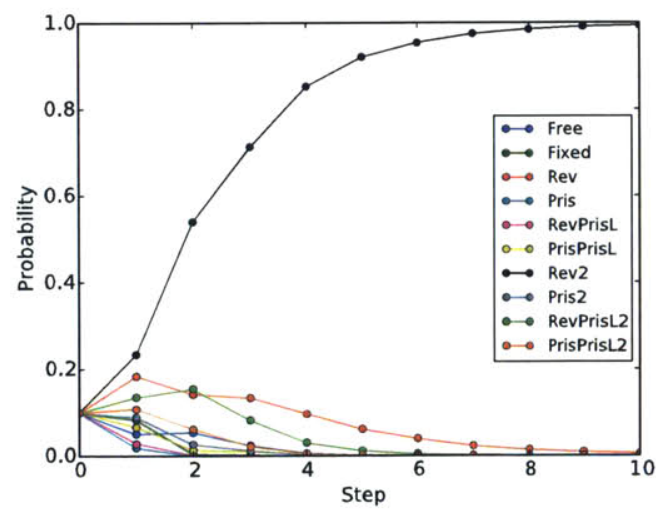

(e) Revolute 2 - Racing

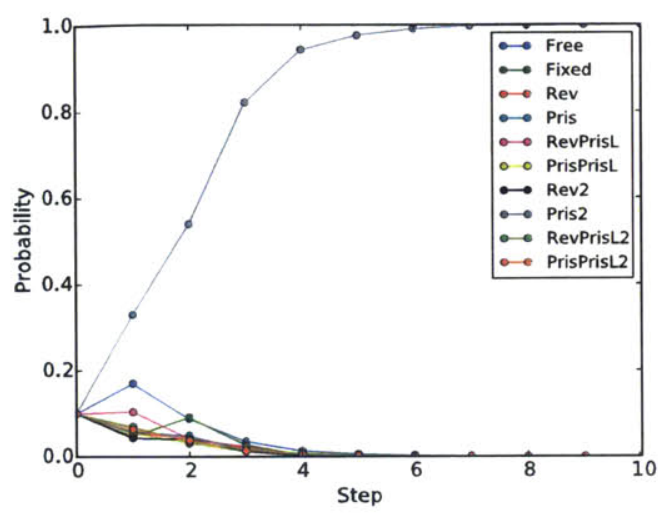

(b) Prismatic 2 - Random

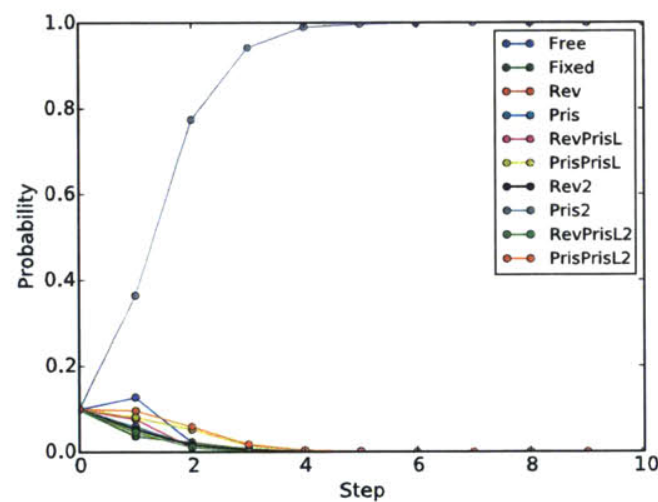

(d) Prismatic 2 - Entropy

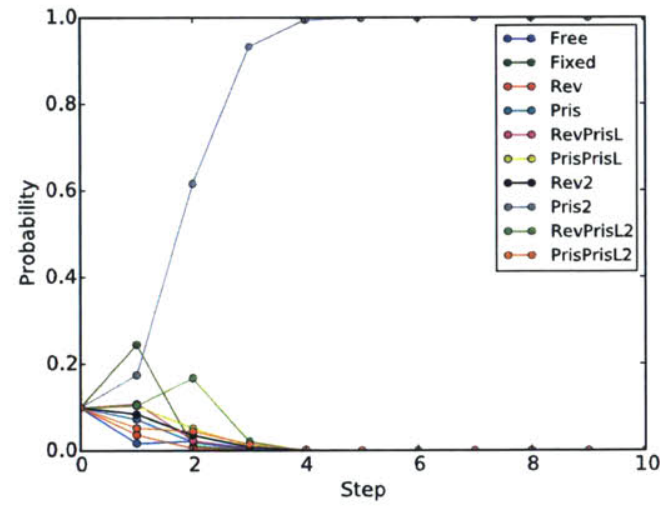

(f) Prismatic 2 - Racing

Figure 3-9: Filter convergence and random vs. entropy-based vs. statistical racing action selection from Revolute 2 and Prismatic 2 models. 


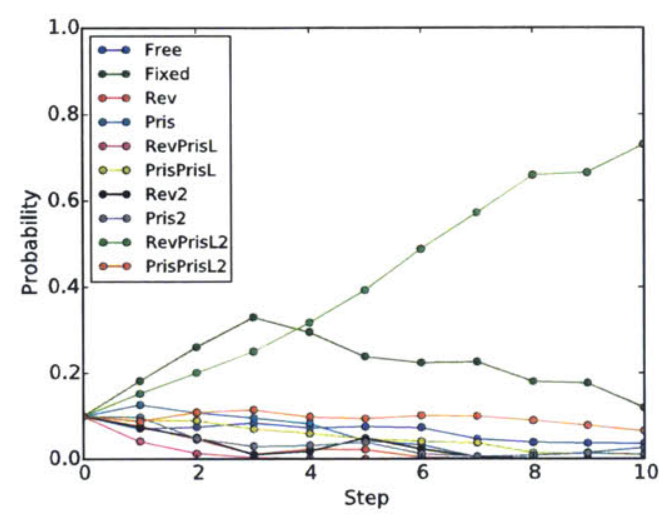

(a) Latch 12 - Random

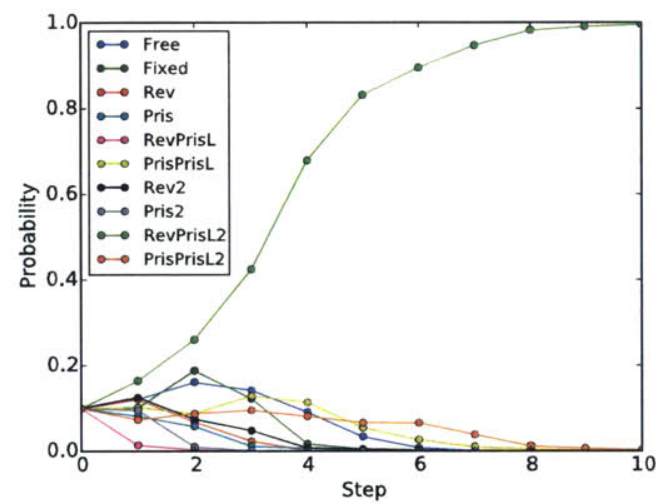

(c) Latch 12 - Entropy

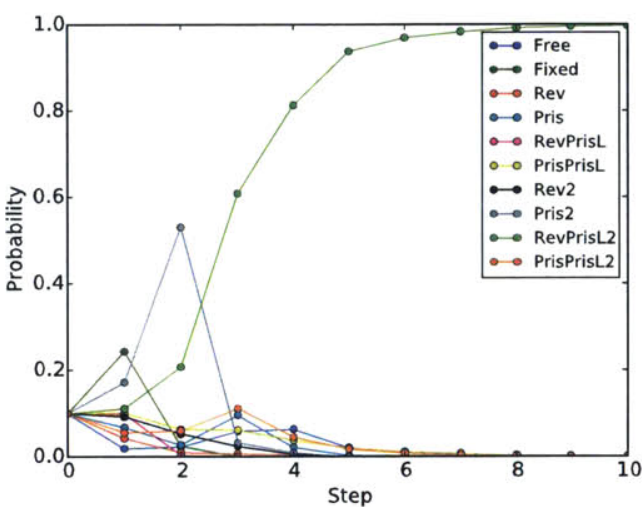

(e) Latch 12 - Racing

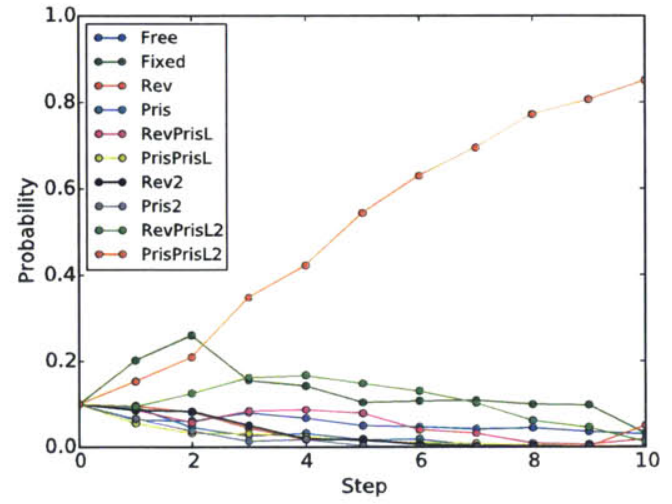

(b) Latch 22 - Random

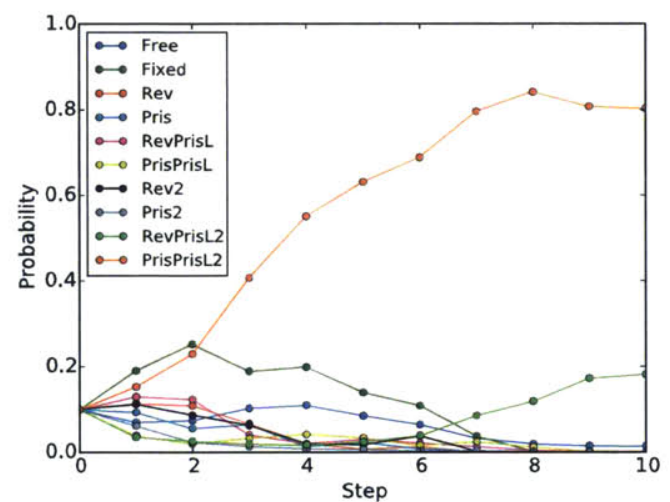

(d) Latch 22 - Entropy

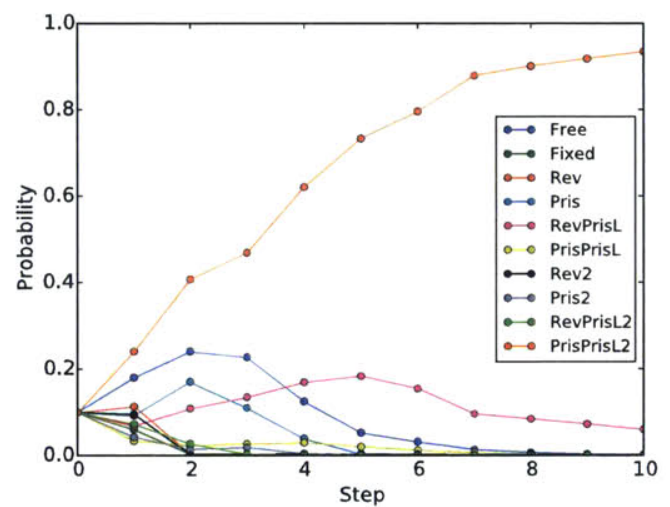

(f) Latch 22 - Racing

Figure 3-10: Filter convergence and random vs. entropy-based vs. statistical racing action selection from Latch 12 and Latch 22 models. 


\begin{tabular}{lcc}
\hline Action-selection Type & P after 5 steps & P after 10 steps \\
\hline Random & 0.70 & 0.89 \\
Entropy & 0.84 & 0.97 \\
Racing & 0.87 & 0.99 \\
\hline
\end{tabular}

Table 3.4: Averaged over 100 experiments per action selection type, the probability of most likely model-parameter pair after five actions and after ten actions for the random, entropy-minimizing, and statistical racing action-selection techniques.

the incorrect model-parameter pair, the likelihood given to that hypothesis could be higher than that given to the correct one. This problem is unlikely to occur on every step of an experiment. However, over numerous experiments, the problem can occur frequently enough to lead to an error. Yet, in $98 \%$ of the experiments, the filter is able to correctly identify the mechanism type and parameters.

Interestingly, despite its heuristic nature, the racing strategy frequently performs as well as or better than the entropy strategy. To explore these strategies further, Table 3.4 shows the average probability over all 300 experiments in the most likely model-parameter set after five actions and after ten actions. First, the active actionselection strategies clearly outperform random as expected. Second, the statistical racing technique slightly outperforms entropy-minimization in these experiments and reaches nearly the same probability values as random in half the steps.

Furthermore, Table 3.5 shows the average running time of a filter step over the 300 experiments for the three action-selection strategies. A filter step includes the action selection and a number of other parts, including the belief update, which are identical for each action-selection strategy. The running times for the random and racing strategies are nearly identical. The running time for the entropy-based technique is approximately 21 times longer than the others. Taking the information presented in these two tables together, statistical racing seems to be a clear winner in terms of the number of actions required for the belief to converge to the correct hypothesis and time complexity under which those actions are chosen.

Finally, the fact that statistical racing frequently slightly improves performance over entropy minimization suggests that minimizing entropy may not be the proper metric for the mechanism estimation problem. Interestingly, racing also appears to 


\begin{tabular}{lc}
\hline Action-selection Type & Average time per step [s] \\
\hline Random & 0.27 \\
Entropy & 5.64 \\
Racing & 0.27 \\
\hline
\end{tabular}

Table 3.5: Averaged over 100 experiments per action selection type, the running time of a step of the filter for the random, entropy-minimizing, and statistical racing action-selection techniques.

\begin{tabular}{lc}
\hline Action-selection Type & Errors (out of 100) \\
\hline Random & 4 \\
Entropy & 3 \\
Racing & 0 \\
\hline
\end{tabular}

Table 3.6: Out of 100 experiments per action selection type, the number of misclassification errors for the random, entropy-minimizing, and statistical racing actionselection techniques.

cause less misclassification errors than the other strategies as shown in Table 3.6. Racing's performance may result from only considering two likely states and disregarding all of the other potentially unlikely states. This focus may reduce the set of plausible hypotheses down to only one winning model-parameter pair more quickly than entropy-based selection which considers the entire distribution at each step.

\subsection{Discussion}

Overall, the discrete Bayesian filter correctly distinguishes the mechanism types considered, including two latching mechanisms which exhibit different constraints in different parts of their configuration spaces. Moreover, action selection based on decreasing belief-state entropy significantly decreases the number of required actions to gather the same information. The filter works in a significantly larger state-space and the time complexity of the active action selection is mitigated by the introduction of a heuristic statistical racing technique. In summary, we demonstrate in simulation that using racing action selection allows the filter to quickly, relative to randomly choosing actions, determine the correct type and parameters of the mechanism of interest with $98 \%$ accuracy. 


\subsubsection{Limitations}

Although applying a discrete Bayesian filter to this problem shows strong results and potential improvements, obvious limitations exist. 10 model-parameter pairs do not densely cover the space of possible mechanism parameters even if restricted to the given mechanism types. Many more possible mechanism types could exist with potentially larger state spaces. Inevitably, time complexity, even with the improvements shown, will continue to limit the size of the space addressable by this method.

To effectively increase the model space and estimation accuracy, we need to move to a different method than the simple discretization presented in this chapter. The current approach becomes computationally intractable for large state spaces due to the exponential growth is number of discrete states. Furthermore, experiments show estimation error due to coarse discretization needed because of computational complexity limitations. The following chapters explore alternate representations of the distribution over the state space that will allow computationally feasible extension to much larger hypothesis spaces. 


\section{Chapter 4}

\section{Consequences of Bayesian Filtering for Hypothesis Comparison}

The state space chosen for the mechanism identification problem is a hybrid space. For any representation, the state space is discrete over mechanism types. The dimensionality of the subspace associated with each mechanism is, in general, different. As described in Section 3.2.3, the observation space is the $x-y$ plane. As discussed in Section 3.2.4, any state is mapped to this same observation space regardless of the mechanism type of the state. Figure 4-1 depicts the hybrid state space and mapping to the observation space. States, denoted as green points, from different dimensionality components of the state space are mapped to nominal observations, denoted as magenta points, in one observation space. The different dimensionality components of the state space are associated with different model types, denoted as $M_{i}$. In this chapter, we explore some of the consequences of the structure of this state space and observation space for Bayesian filtering.

\subsection{Continuous State-space, Parametric Filters}

Although the mechanism-type dimension in our state space is discrete, the other dimensions in the mechanism identification problem are continuous. Standard Bayesian filtering techniques for continuous variables, such as the Kalman filter, have update 


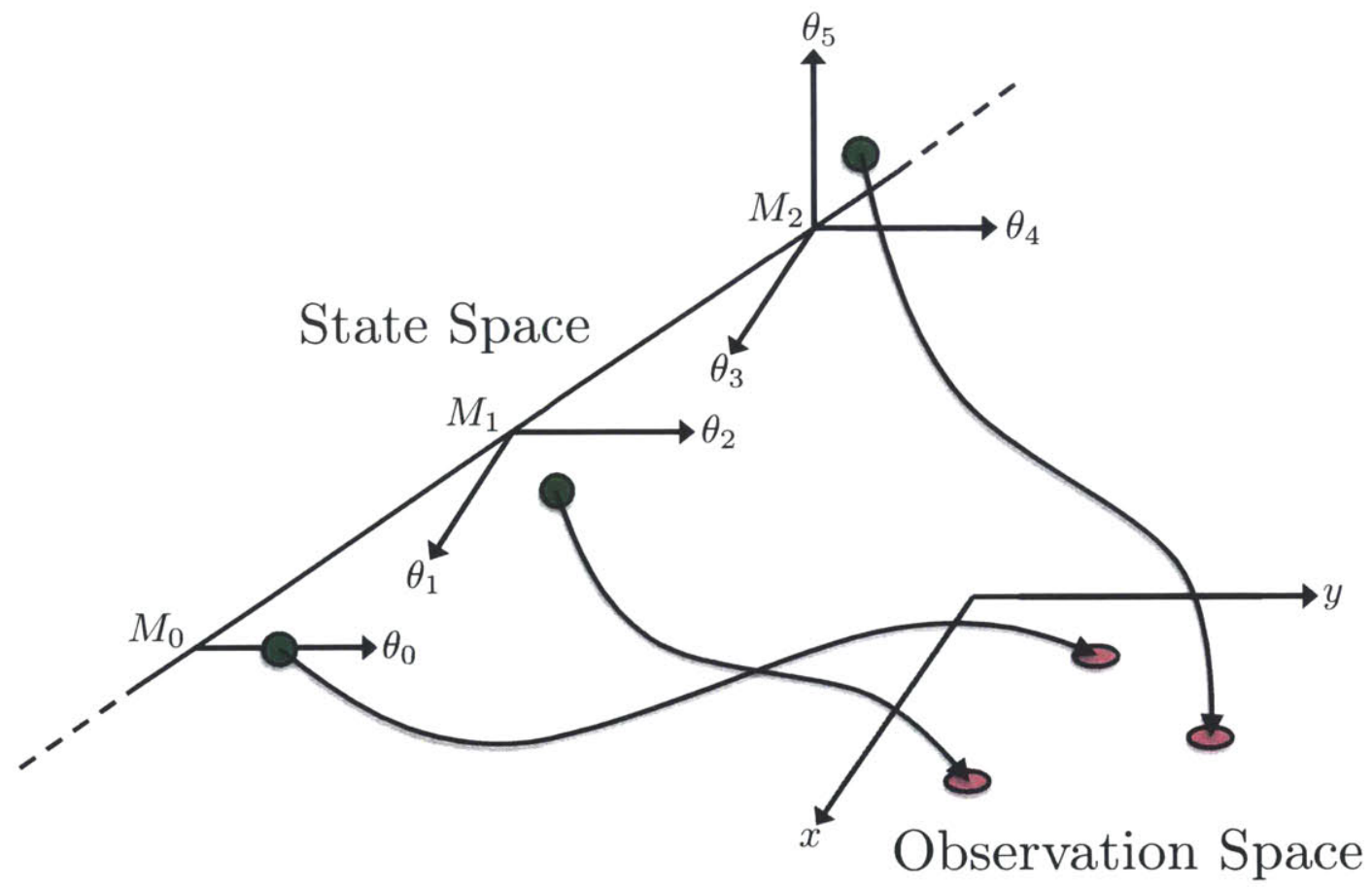

Figure 4-1: The mechanism identification problem is presented as a hybrid state space. The space is discrete over mechanism types, and each mechanism type has an associated, possibly different-dimensionality subspace. Regardless of mechanism type, a state, shown as a green dot, maps to a nominal observation, show as magenta dot, in the observation space. 
algorithms with low computational complexity and strong performance guarantees. However, these techniques each impose certain constraints on the forms of the belief distribution, as well as the transition and observation models. In general, our domain has highly-nonlinear transition and observation models. Extended or unscented Kalman filters can be used to handle these nonlinearities $[22,7]$. However, all of these methods assume Gaussian distributions for the belief. If the belief distributions in the mechanism identification context are unimodal or can be accurately approximated as unimodal, these filters may be appropriate.

A simple example gives insight into the types of distributions to expect in our problem. Consider attempting to estimate the parameters, radius and hinge position, of a door. For simplicity, assume the radius is known. Also, assume initially that the prior over hinge positions is uniform such that a unimodal distribution could approximate the belief. Attached to the handle of the door, the robot's gripper attempts to move forward and receives an observation forward and slightly to the right of the initial pose of the gripper. The posterior distribution over hinge positions would now show high probability for doors hinged toward the right and left of the robot but low probability for doors hinged in forward or backward. After this one step, the belief distribution is bimodal.

This example is only one of many possible multimodal distributions which can occur even in the space of mechanisms considered in Chapter 3, let alone the various other mechanism types which the robot may encounter. Thus, unimodal distributions are likely inappropriate in general for this problem.

Other parametric forms such as Gaussian mixtures can represent multimodal distributions. However, the parametric form and associated algorithm that would be appropriate for all priors and sequences of actions and observations in this domain is unknown. Our discrete filter could represent arbitrary distributions and nonlinear transitions and observations but was intractable for large state spaces. Instead, we choose a representation and filtering algorithm which, like the discrete filter, can represent arbitrary distributions but can handle large state spaces. We discuss the use of a particle filter in Section 5 to meet these requirements. 


\subsection{Unintuitive Results of Bayesian Filtering in a Hybrid Space}

Applying standard Bayesian filtering in our hybrid space can produce unintuitive results. Some arise from modeling choices while others derive directly from the mathematics of Bayesian inference. This unintuitive behavior may be potentially undesirable and accounts for most of the misclassification errors shown in the Section 5.4 when using the particle filter. However, the phenomenon discussed in the following sections is not restricted to a particular filtering technique or belief representation. Moreover, these phenomenon all speak to the importance of highly informative actions and observations. Methods to explicitly seek information by actively selecting actions were discussed in Section 3.3.

\subsubsection{Transition Model Quality}

The quality of the filter's transition models for each mechanism type can easily affect the likelihoods given to that type's hypotheses. If a mechanism model poorly predicts the mechanisms actual transitions, the probability mass associated with a mechanism type will likely be far from the observation from the world. Thus, hypotheses of a different mechanism class may increase in likelihood if they produce nominal observations near the true observation on that step. This possibility is especially problematic when two mechanism types behave similarly in a region of the observation space. With a sufficiently poor model of one of the two types, hypotheses of the other type may become more probable if the chosen actions only produce observations in the region where both types act similarly.

For example, consider filtering over a space of one revolute mechanism and one prismatic mechanism while the robot truly interacts with the revolute mechanism. Let the prismatic mechanism be tangent to the revolute mechanism at the origin such that the two mechanisms act similarly in this region of the observation space. Assume the filter's prior is equal for each model. If the filter's models of the mechanisms are perfect 
and an action is taken which would move the two mechanisms to a configuration that would produce similar observations, then incorporating the true observation into the filter would assign reasonable probability to both hypotheses.

Figure 4-2(a) shows such a scenario. As shown, the blue lines represent all possible noiseless, nominal observations for each mechanism type. The start state in the observation space is at the origin as denoted by the green dot. The robot performs an action denoted by the black arrow. In the real world, the two mechanisms would move to the end states represented in the observation space as red dots. However, the robot is interacting with the revolute mechanism and could perhaps receive the noisy observation denoted by the magenta dot. With a Gaussian observation model, the nominal observations of both of these hypotheses would have some likelihood, but the revolute hypothesis would have a higher observation likelihood and thus become more likely relative to the prismatic model.

Instead, consider a scenario where the prismatic transition model very closely reflects the robot's interactions with the world but the revolute transition model very poorly predicts transitions due to unmodeled details. Because the true mechanisms act similarly in this region of the observation space, if the robot performs the same action on the true revolute joint and receives the same observation, the revolute observation likelihood would be likely lower than that of the prismatic model. Figure 4-2(b) shows this scenario and is drawn identically to Figure 4-2(a). The prismatic transition prediction is not perfect but still results in a nominal observation near the true observation. However, the revolute model poorly predicts its nominal observation very far from the true one. The observation likelihood ratio favors the prismatic model, and it becomes more likely despite that the robot is truly interacting with the revolute joint. Again, this results directly from a good quality prismatic model and a poor quality revolute model in a scenario where the two true mechanisms behave similarly. In terms of correctly identifying the model class, this behavior will lead to poor performance. This phenomenon partially accounts for the confusion of revolute joints for prismatic joints discussed in Section 5.4. This result occurs because mechanisms of each type can act similarly near the origin of the observation space and our revolute 


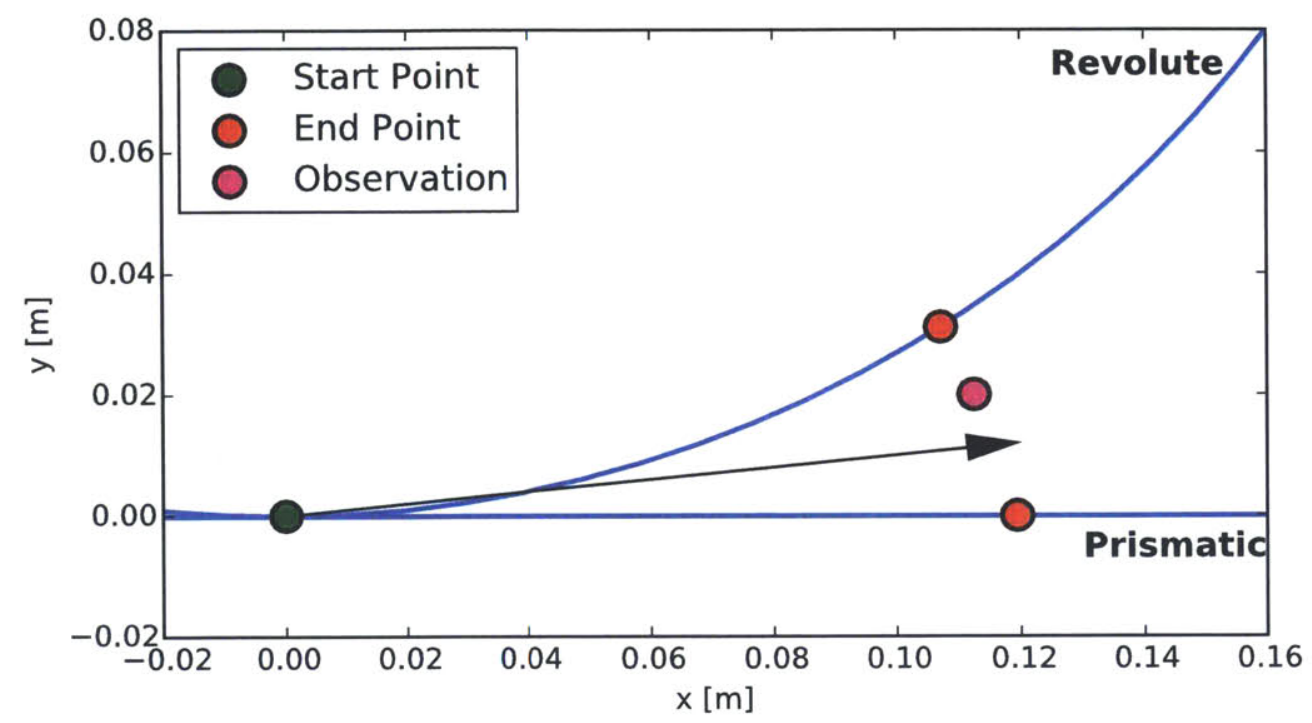

(a) Perfect models for revolute and prismatic

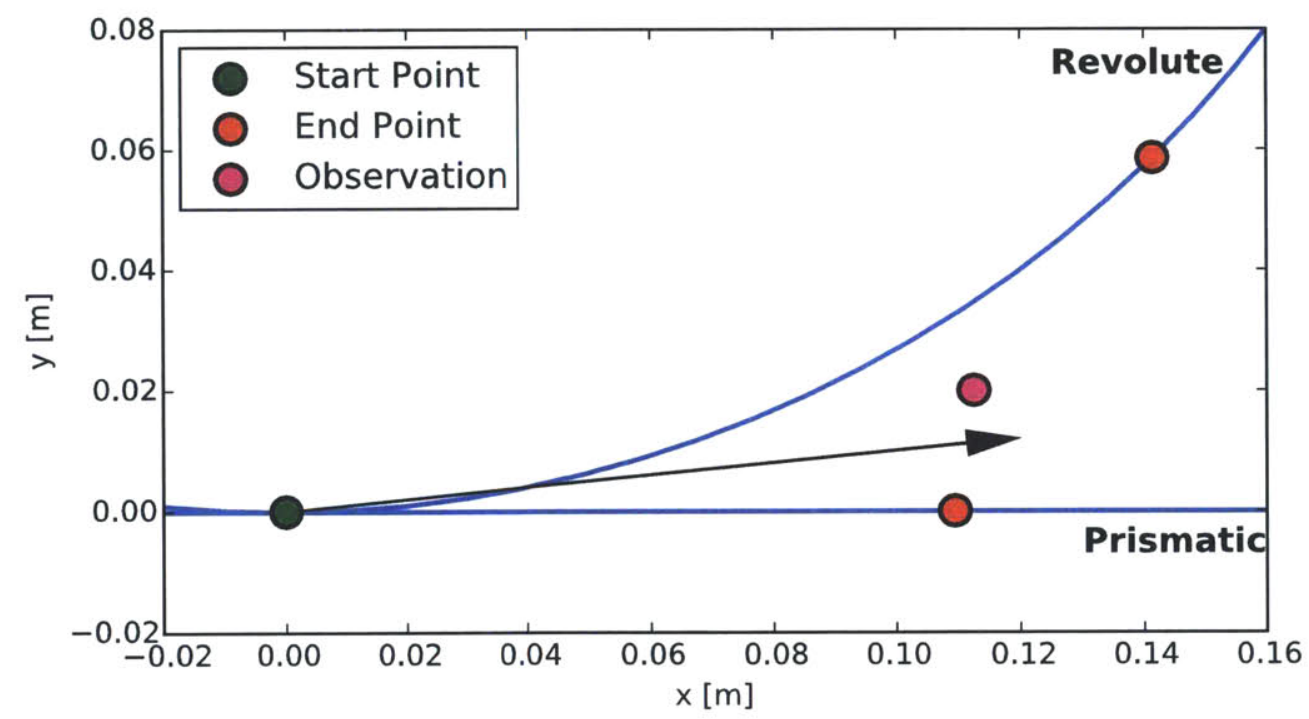

(b) Poor revolute model and good prismatic model

Figure 4-2: Comparison of the effect of model quality on the observation likelihood ratio between a revolute and prismatic hypothesis tangent to the revolute hypothesis at the origin. These hypotheses act similarly in this region of the observation space. All possible noiseless, nominal observations of each mechanism are represented as a labeled blue line. The start state at the origin of the observation space is shown as a black dot with green outline. The action taken is denoted as a black arrow. The end states in the observation space assuming each type are shown as black dots with red outlines. The observation is shown as a black dot with magenta outline. a) The filter models of the mechanisms are perfect b) The filter has a good quality model of the prismatic mechanism but a poor quality model of the revolute mechanism. 
model predicts true revolute motion poorly.

The modeling problem can affect all filter types. However, because our application requires filtering over a hybrid space with different models, the comparative quality of those models affects accuracy of the belief updates. How to ensure that all mechanism types are modeled equally well is unclear. A possible approach could be to rate the quality of a model by comparing its predicted observation to a measured observation from the real world for different states and actions. The model quality problem reinforces the need to choose actions which help clearly distinguish mechanisms such that the variation in prediction quality becomes less significant to the estimates.

\subsubsection{Bayesian Occam's Razor}

In Chapter 28 of his book, MacKay [12] carefully explains the concept of the Bayesian Occam's razor. Simply stated, in model comparison, Bayesian inference will assign more likelihood to the simplest model given that the data can be explained equally well by all models. This phenomenon plays a key role in mechanism identification, because we are trying to choose among hypotheses of significantly different complexity.

Throughout this discussion, we are interested in $P(M \mid O)$ where $M$ is a hypothesis and $O$ is an observation or set of observations. For simplicity, we will use slightly different notation from that introduced in Section 1.1.1. In the following, $\theta$ will refer to a generic state vector. With basic manipulation,

$$
\begin{aligned}
P(M \mid O) & =\int_{\theta} P(M, \Theta \mid O) d \theta \\
& =\left(\frac{1}{Z}\right) \int_{\theta} P(O \mid M, \Theta) P(M, \Theta) d \theta \\
& =\left(\frac{1}{Z}\right) \int_{\theta} P(O \mid M, \Theta) P(M) P(\Theta \mid M) d \theta
\end{aligned}
$$


where

$$
\begin{aligned}
Z & =P(O) \\
& =\sum_{i} \int_{\theta} P\left(O \mid M=m_{i}, \Theta\right) P\left(M=m_{i}, \Theta\right) d \theta
\end{aligned}
$$

is a normalizer that does not generally need to be calculated. Forms of Equation 4.1 will appeared repeatedly in our following explanations.

\section{Given a more ambiguous observation}

In our context, consider filtering over the space of two hypotheses, a free mechanism and a prismatic mechanism aligned with the $\mathrm{x}$-axis passing through the origin of the observation space. The observation space is $(x, y)$ in the plane. Let the first model $M_{1}$ be a two-dimensional free model with configuration variables $\theta_{1}$ and $\theta_{2}$ each in $[-1,1]$. Let the second model $M_{2}$ be a one-dimensional prismatic model with configuration variable $\theta_{3}$ in $[-1,1]$. For the free model, the configuration $\left(\theta_{1}, \theta_{2}\right)=(x, y)$. For the prismatic model, the configuration $\theta_{3}=x$. Recall that the prismatic model passes through $(0,0)$ with a fixed $y$ coordinate of 0 . For simplicity, the following equations will substitute the configuration variables for the corresponding variables in observation space.

Assume that initially each hypothesis is equally likely. Specifically,

$$
P(M=1)=P(M=2)=\frac{1}{2} .
$$

Also assume that the variable distributions are uniform, given each model. Specifcally,

$$
\begin{aligned}
X, Y \mid M & =1 \sim \operatorname{UnifORM}([-1,1],[-1,1]) \\
X \mid M & =2 \sim \operatorname{UNIFORM}([-1,1]) .
\end{aligned}
$$


Thus,

$$
\begin{array}{r}
P(X, Y \mid M=1)=\frac{1}{4} \\
P(X \mid M=2)=\frac{1}{2} .
\end{array}
$$

Let the observation model be Normal about the nominal observation for both models with a covariance matrix $\Sigma_{o b s}$. For this example, assume $\Sigma_{o b s}$ is the $2 \times 2$ identity matrix. Then, the observation model is

$$
\begin{array}{r}
O \mid M=1, X=x, Y=y \sim \operatorname{NormaL}\left((x, y), \Sigma_{o b s}\right) \\
O \mid M=2, X=x \sim \operatorname{NormaL}\left((x, 0), \Sigma_{o b s}\right) .
\end{array}
$$

Imagine we get an observation $o=(0.1,0.1)$, and we want to know the posterior over model types $P(M \mid O)$. Then, for $M_{1}$,

$$
\begin{aligned}
P(M=1 \mid O=(0.1,0.1))= & \iint_{x, y} P(M=1, X=x, Y=y \mid O=(.1, .1)) d x d y \\
= & \left(\frac{1}{Z}\right) \iint_{x, y} P(O=(0.1,0.1) \mid M=1, X=x, Y=y) \\
& \cdot P(M=1, X=x, Y=y) d x d y \\
= & \left(\frac{1}{Z}\right) \iint_{x, y} P(O=(0.1,0.1) \mid M=1, X=x, Y=y) \\
& \cdot P(M=1) P(X=x, Y=y \mid M=1) d x d y \\
= & \left(\frac{1}{Z}\right) \iint_{x, y}\left(\frac{1}{2}\right)\left(\frac{1}{4}\right) \\
& \cdot P(O=(.1, .1) \mid M=1, X=x, Y=y) d x d y \\
= & \left(\frac{1}{Z}\right)\left(\frac{1}{2}\right)\left(\frac{1}{4}\right) \\
& \left.\cdot \iint_{x, y} \text { NormaLPDF }(.1, .1) ;(x, y), \Sigma_{o b s}\right) d x d y, \quad(4.5)
\end{aligned}
$$


where

$$
\begin{aligned}
Z= & P(O=(0.1,0.1)) \\
= & \iint_{x, y} P(O=(0.1,0.1) \mid M=1, X=x, Y=y) P(M=1, X=x, Y=y) d x d y \\
& +\int_{x} P(O=(0.1,0.1) \mid M=2, X=x) P(M=2, X=x) d x
\end{aligned}
$$

Similarly, for $M_{2}$,

$$
\begin{aligned}
P(M=2 \mid O=(0.1,0.1))= & \int_{x} P(M=2, X=x \mid O=(.1, .1)) d x \\
= & \left(\frac{1}{Z}\right) \int_{x} P(O=(0.1,0.1) \mid M=2, X=x) \\
& \cdot P(M=2, X=x) d x \\
= & \left(\frac{1}{Z}\right) \int_{x} P(O=(0.1,0.1) \mid M=2, X=x) \\
& \cdot P(M=2) P(X=x \mid M=2) d x \\
= & \left(\frac{1}{Z}\right) \int_{x}\left(\frac{1}{2}\right)\left(\frac{1}{2}\right) \\
& \cdot P(O=(0.1,0.1) \mid M=2, X=x) d x \\
= & \left(\frac{1}{Z}\right)\left(\frac{1}{2}\right)\left(\frac{1}{2}\right) \\
& \cdot \int_{x} \text { NormaLPDF }\left((0.1,0.1) ;(x, 0), \Sigma_{o b s}\right) d x .
\end{aligned}
$$

Referring to Equations 4.5 and 4.6, we are interested in

$$
P(M=1 \mid O=(0.1,0.1))=\frac{A}{A+B}
$$

and

$$
P(M=2 \mid O=(0.1,0.1))=\frac{B}{A+B}
$$


where

$$
\begin{aligned}
A & =\left(\frac{1}{2}\right)\left(\frac{1}{4}\right) \iint_{x, y} \operatorname{NoRMALPDF}\left((.1, .1) ;(x, y), \Sigma_{o b s}\right) d x, d y \\
B & =\left(\frac{1}{2}\right)\left(\frac{1}{2}\right) \int_{x} \operatorname{NoRMALPDF}\left((.1, .1) ;(x, 0), \Sigma_{o b s}\right) d x
\end{aligned}
$$

Using the numbers in this example, we find that $A=0.058$ and $B=0.068$, which gives $P(M=1 \mid O=(0.1,0.1))=0.46$ and $P(M=2 \mid O=(0.1,0.1))=0.54$. Although the observation $o=(0.1,0.1)$ does not fall directly on the locus of nominal observations for the prismatic model, the probability of the lower-dimensional prismatic model increases.

Because the prismatic model is only one dimensional while the free model is two dimensional, the probability mass for the prismatic model is more concentrated than that of the free. Because each hypothesis generates observations with the same variance, states from each model that produce corresponding identical nominal observations would produce identical observation likelihoods for $o$. However, given our uniform prior distributions, the likelihood of the states from the different hypotheses would be different. Namely, the likelihood from the higher-dimensional model would be lower. After receiving an observation reasonably consistent with both models, the hypothesis probability for the higher-dimensional model would decrease. In our context, we would choose the lower dimensional hypothesis as our estimate although the observation is reasonably consistent with both.

Figure 4-3 shows a graphical representation of an example nearly identical to the one discussed above. For simplicity, the depicted example only differs from the explanation above in the observation model. Consider an observation model simpler than the Gaussian observation model. Assume

$$
P(O \mid M, \Theta)=P\left(O \mid O^{*}\right)= \begin{cases}\frac{1}{\pi r^{2}}, & \text { if }\left\|o-o^{*}\right\|<r \\ 0, & \text { otherwise }\end{cases}
$$

where $o^{*}$ is the nominal observation associated with a state and $o^{*}=f(m, \theta)$ is the 


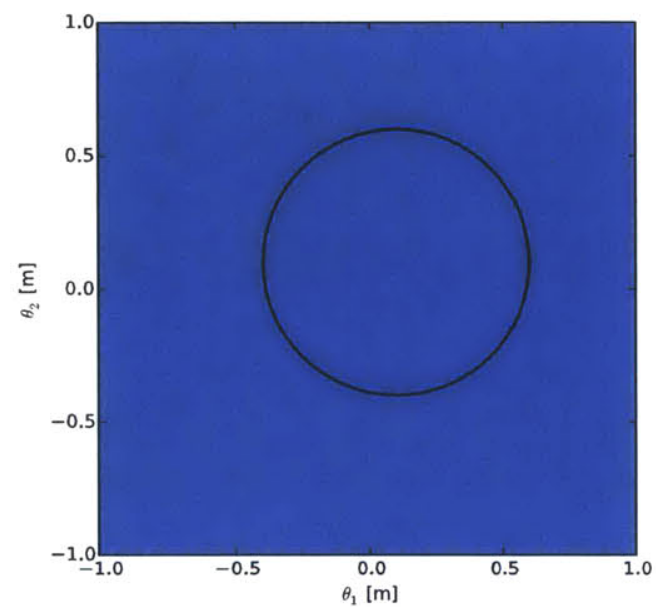

(a) Free - C-space

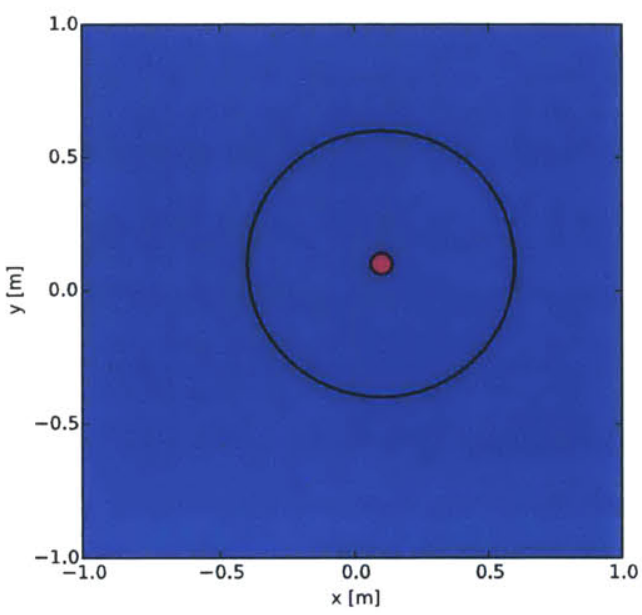

(c) Free - O-space

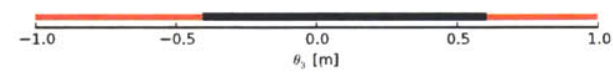

(b) Prismatic - C-space

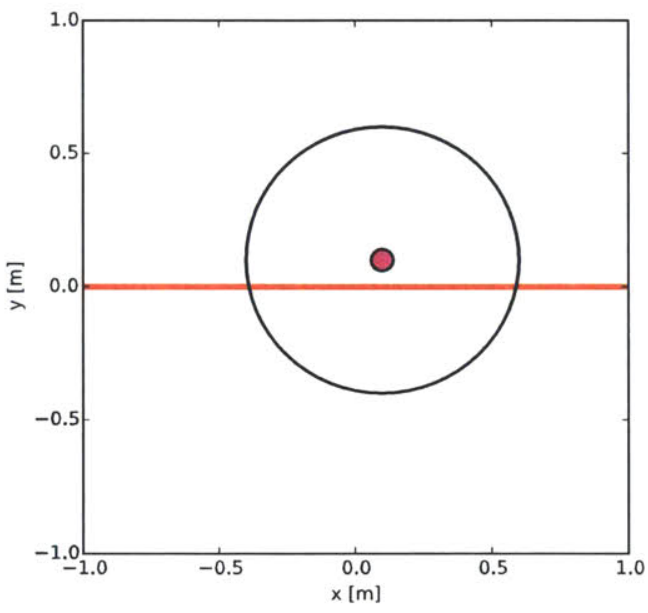

(d) Prismatic - O-space

Figure 4-3: Visual depiction of the Bayesian Occam's razor phenomenon for a scenario of comparing a free hypothesis and a prismatic hypothesis given an observation close to $y=0$. Blue denotes regions of lower probability, while red denotes regions of higher probability. The black circles and black line denote regions where states or their corresponding nominal observations would assign nonzero probability to observation o. a) $P(\Theta \mid M)$ shown on the configuration space of the free model. b) $P(\Theta \mid M)$ shown on the configuration space of the prismatic model. c) $P\left(O^{*} \mid M\right)$ shown on the observation space of the free model. d) $P\left(O^{*} \mid M\right)$ shown on the observation space of the prismatic model. 
mapping between them.

The top row of the figure shows $P(\Theta \mid M)$ over the configuration space of the models, whereas the bottom row shows $P\left(O^{*} \mid M\right)$ over the observation space for the two models. The left column depicts the free mechanism, while the right column depicts the prismatic mechanism. The lighter blue color denotes lower probability, whereas the brighter red color denotes higher probability.

Shown in Figure 4-3(c), the world produces an observation $o=(0.1,0.1)$, denoted by a magenta dot, close to but not on top of the locus of nominal observations for the prismatic model. The black circle denotes the region of all $o^{*}$ for which $P\left(O \mid O^{*}\right)$ will be nonzero. The same magenta dot and circle are drawn in Figure 4-3(d). We are interested in the regions of states in configuration space that would yield nominal observations $o^{*}$ that would give $o$ a nonzero likelihood. The regions in observation space can be mapped back into the configuration space of the models. Because of the relationship between the two spaces for the free model, the region is identical in size and position after mapping, as shown in Figure 4-3(a). However, the prismatic model only has nominal observations on a horizontal line in observation space. Where the circle intersects this line denotes the region in the prismatic configuration space that correspond to states that would yield nominal observations $o^{*}$ that would give $o$ a nonzero likelihood. This region is denoted in black covering a section of the configuration space as shown in Figure 4-3(b).

Recall Equations 4.1 through 4.4. The integral in Equation 4.1 has three terms. For both models, $P(M)=0.5$. For the free model, $P(\Theta \mid M=1)=0.25$, whereas for the prismatic model, $P(\Theta \mid M=2)=0.5$. Note that these terms are both constants, and, if we assume that we only integrate over values of $\theta$ in the square, the terms can be moved outside of the integral. Also, note that given the same value of the integral for both models, the prismatic model would be twice as likely as the free model.

Which model has the higher probability after incorporating the observation depends on the relative values of the integral for each model. Because of the chosen observation model, the magnitude of the integral over $\theta$ of $P(O \mid M, \Theta)$ will depend on the ratio of the area of states whose corresponding nominal observations assign $o$ 
nonzero likelihood to the area of the valid state space. For the free model, this ratio is the area of the circle, where the integrand will be nonzero, to the area of the entire square. For the prismatic model, the ratio is the length of the black section of the line to the total length of the line. As shown in the figure, these ratios clearly show that, given $o=(0.1,0.1)$, the integral for the prismatic model is larger than that for the free model. Combined with the values of $P(\Theta \mid M)$, the values of the integrals show that the prismatic model's probability will increase compared to that of the free model, which is the same result from the calculations above.

\section{Given a less ambiguous observation}

Consider, again, the calculations shown above assuming a Gaussian observation model. Assume the observation instead was $o=(0.1,1.5)$. Repeating the same calculations above for this observation gives $A=0.026$ and $B=0.022$, which gives $P(M=1 \mid O=(0.1,1.5))=0.54$ and $P(M=2 \mid O=(0.1,1.5))=0.46$. In this case, the observation is sufficiently unlikely for the prismatic model that the higher-dimensional free model becomes more likely.

To repeat the graphical explanation, again consider the simpler observation model given in Equation 4.7 and an observation $o=(0.5,0.485)$. Figure 4-4 depicts this scenario and is drawn identically to Figure 4-3 explained above. Although in Figure 44(a) the circular region moves compared to the closer observation, the region still entirely overlaps the nonzero probability region of the free model. For the prismatic model, the circular region only slightly overlaps the line in Figure 4-4(d) which maps to a much smaller section of the line in Figure 4-4(b). In this case, the ratio of relevant areas for the free model is identical to the previous explanation. However, the ratio of relevant lengths for the prismatic model is significantly smaller. This change leads to an integral value that offsets the difference in values of $P(\Theta \mid M)$ between the models which allows the probability of the free model to increase relative to that of the prismatic model.

In summary, these results suggests that the filter's inference is affected by the dimensionality of the various hypotheses. A lower-dimensional model can become 


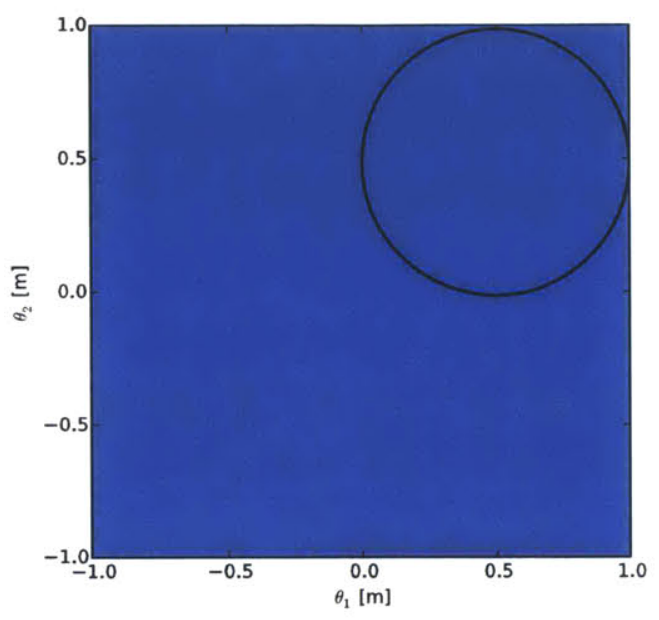

(a) Free - C-space

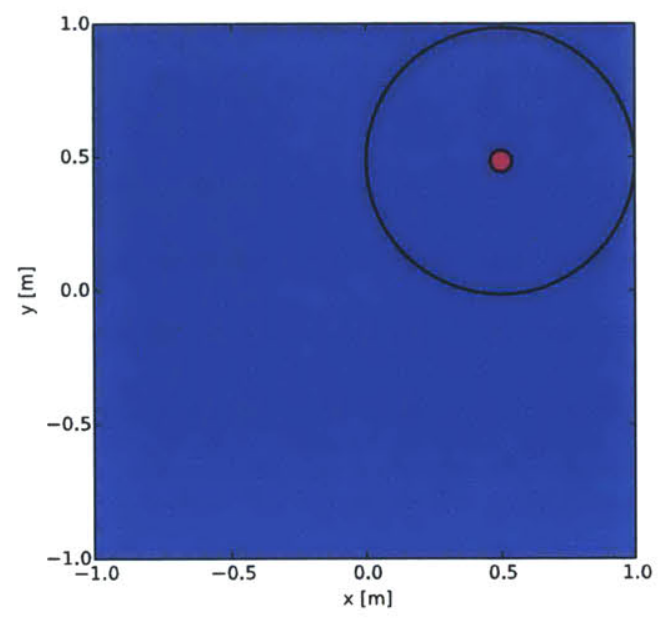

(c) Free - O-space

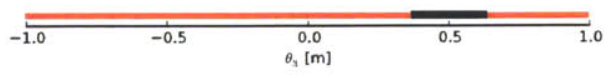

(b) Prismatic - C-space

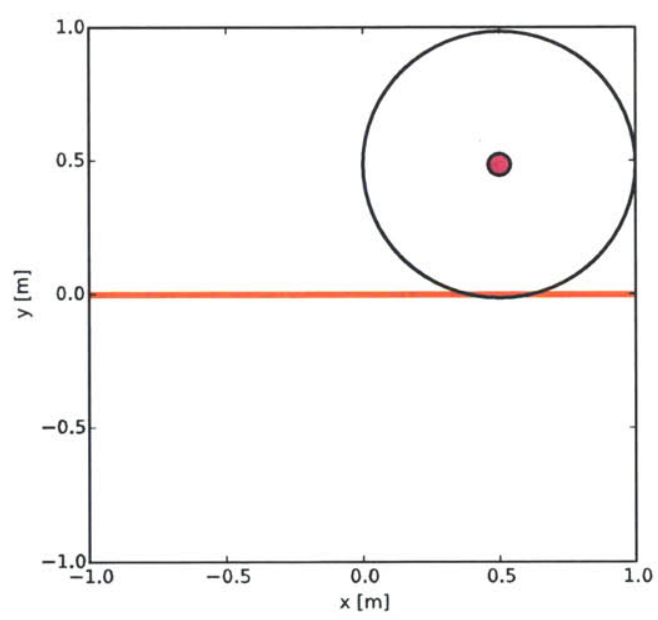

(d) Prismatic - O-space

Figure 4-4: Visual depiction of the Bayesian Occam's razor phenomenon for a scenario of comparing a free hypothesis and a prismatic hypothesis given an observation far from $y=0$. Blue denotes regions of lower probability, while red denotes regions of higher probability. The black circles and black line denote regions where states or their corresponding nominal observations would assign nonzero probability to observation o. a) $P(\Theta \mid M)$ shown on the configuration space of the free model. b) $P(\Theta \mid M)$ shown on the configuration space of the prismatic model. c) $P\left(O^{*} \mid M\right)$ shown on the observation space of the free model. d) $P\left(O^{*} \mid M\right)$ shown on the observation space of the prismatic model. 
more probable compared to a higher dimensional model even given an observation that intuitively appears reasonable for both. Instead, the filter must receive observations sufficiently unlikely for the lower dimensional model to choose the other. This conclusion initially appears unintuitive but follows directly from the mathematics of Bayesian inference and is not an error. However, the result does suggest that the filter will have a tendency to choose lower dimensional hypotheses.

\subsubsection{Projection}

A third phenomenon, which we call projection, occurs for similar reasons as the Bayesian Occam's razor and leads to similar results. In general, the state and observation spaces in an inference problem will be different. Frequently, states from the state space are mapped to the observation space for comparison with the true observation. This mapping projects the belief from the state space to the observation space.

Two models of the same dimensionality can project differently from state to observation space. If the mapping of one constricts the probability mass to a smaller volume of the observation space than the other, an observation consistent with both models can lead to more likelihood given to the more constricted model for similar reasons as described above.

\section{Given a more ambiguous observation}

Consider filtering to compare a free mechanism with a "scaled" free mechanism. This scaled mechanism is not treated in other parts of this thesis but is used here for illustrative purposes. A physical example of such a scaled free mechanism would be a pantograph. Just as described in Section 4.2.2, the observation space is $x-y$ plane. Let the first model $M_{1}$ be a two-dimensional free model with configuration variables $\theta_{1}$ and $\theta_{2}$ each in $[-1,1]$. Again, when mapping from configuration to observation space, the configuration $\left(\theta_{1}, \theta_{2}\right)=(x, y)$. Let the second model $M_{2}$ be a two-dimensional free model with configuration variable $\theta_{3}$ and $\theta_{4}$ each in $[-1,1] . M_{2}$ 
is called a scaled free model because the mapping from configuration to observation space is $\left(\theta_{3}, \theta_{4}\right)=(2 x, 2 y)$.

Again, assume that initially each hypothesis is equally likely

$$
P(M=1)=P(M=2)=\frac{1}{2}
$$

and that the variable distributions are uniform, given each model.

$$
\begin{aligned}
& \Theta_{1}, \Theta_{2} \mid M=1 \sim \operatorname{UNIFORM}([-1,1],[-1,1]) \\
& \Theta_{3}, \Theta_{4} \mid M=2 \sim \operatorname{UNIFORM}([-1,1],[-1,1]),
\end{aligned}
$$

and, thus,

$$
P\left(\Theta_{1}, \Theta_{2} \mid M=1\right)=P\left(\Theta_{3}, \Theta_{4} \mid M=2\right)=\frac{1}{2} .
$$

Figure 4-5 illustrates the result of receiving an observation close to the origin of the observation space $o=(0.1,0.1)$. Again, the observation model used is given in Equation 4.7. Just as before, the top row of the figure shows $P(\Theta \mid M)$ over the configuration space of the models, whereas the bottom row shows $P\left(O^{*} \mid M\right)$ over the observation space for the models. The left column depicts the free mechanism, while the right column depicts the scaled free mechanism. The darkest blue color denotes zero probability. The lighter blue color denotes low but nonzero probability, whereas the brighter orange color denotes higher probability.

Also just as in the previous figures, the observation is denoted by a magenta dot in the bottom row. The black circles in the bottom row denote the region of all $o^{*}$ for which $P\left(O \mid O^{*}\right)$ will be nonzero. The same magenta dot and circle are drawn on Figure 4-5(c) and (d). We are again interested in the regions of states in configuration space that would yield nominal observations $o^{*}$ that would give $o$ a nonzero likelihood. We map the regions in observation space back into the configuration space of the models. Because of the relationship between the two spaces for the free model, the region is identical in size and position after mapping as shown in Figure 4-5(a). 


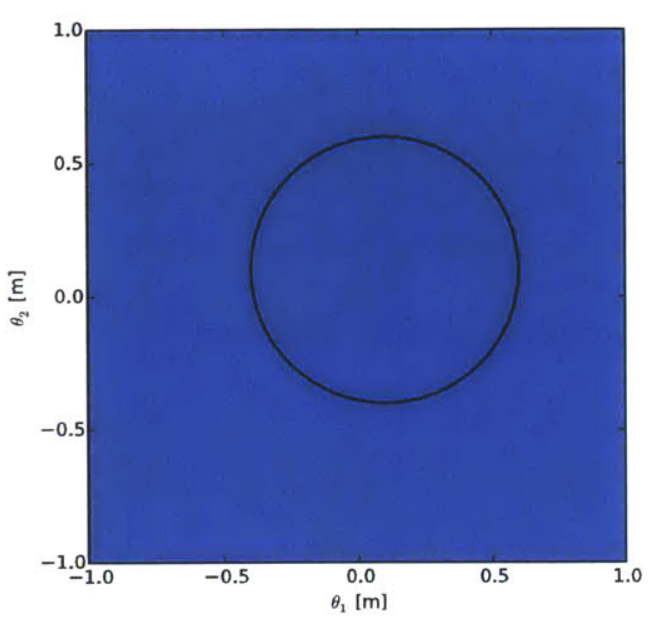

(a) Free - C-space

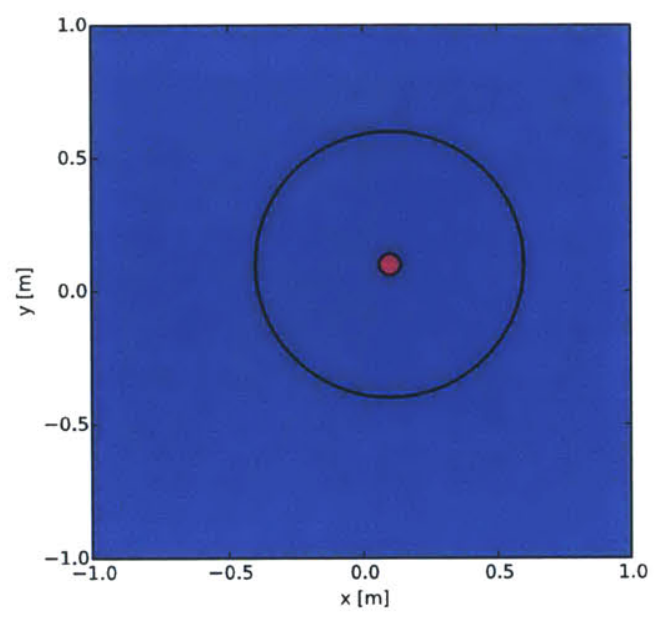

(c) Free - O-space

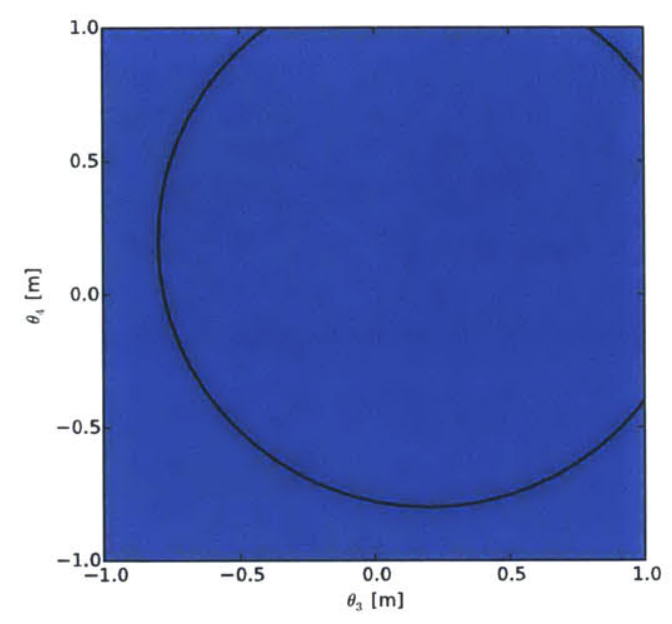

(b) Prismatic - C-space

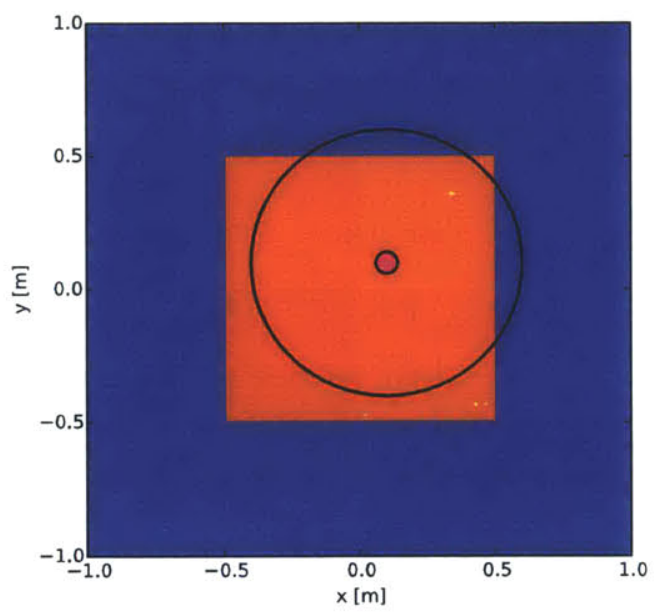

(d) Prismatic - O-space

Figure 4-5: Visual depiction of the projection phenomenon for a scenario of comparing a free hypothesis and a scaled free hypothesis given an observation close to the origin of the observation space. The darkest blue denotes regions of zero probability. The lighter blue denotes regions of lower probability, while orange denotes regions of higher probability. The black circles denote regions where states or their corresponding nominal observations would assign nonzero probability to observation $o$. a) $P(\Theta \mid M)$ shown on the configuration space of the free model. b) $P(\Theta \mid M)$ shown on the configuration space of the scaled free model. c) $P\left(O^{*} \mid M\right)$ shown on the observation space of the free model. d) $P\left(O^{*} \mid M\right)$ shown on the observation space of the scaled free model. 
However, the the scaled free model's spaces are related by a factor of two. Therefore, the region denoted by the black circle in the configuration space is twice the radius with a center twice as far from the origin as shown in Figure 4-5(b).

We repeat the examination of Equation 4.1 for this example. Recall Equations 4.8 and 4.9. Because both models have the same dimensionality, two of the three terms in the integral of Equation 4.1 are constant and equal. Again, if we assume that we only integrate over values of $\theta$ in the square, the constant terms can be moved out of the integral. The relative values of the integral for each model type will tell which model's probability will increase relative to the other. As before, the chosen observation model simplifies the integral to an area comparison. The magnitude of the integral over $\theta$ of $P(O \mid M, \Theta)$ will depend on the ratio of the area of states whose corresponding nominal observations assign $o$ nonzero likelihood to the area of the valid state space. For each figure in the top row of Figure 4-5, this ratio is the area inside the circle contained in the square over the area of the square. Clearly, these figures depict a much larger integral value for the scaled free model although the observation initially appears explainable by both models. Overall, the probability of the scaled free model will increase relative to the probability of the free model.

\section{Given a less ambiguous observation}

Consider instead receiving $o=(0.75,0.75)$, an observation far from the origin compared to the observation discussed above. Figure 4-6 illustrates this example and is drawn the same way as the previous figures. Following the same logic as before, we see that the ratio of the areas inside the circles contained in the square over the area of the squares for the two different models will give us the values of the integral for the two models. The ratio of these values will give us the ratio of their probabilities after incorporating the observation. In this case, both circles in configuration space only partially overlap the square region of nonzero-probability states. However, the scaled free model has a smaller overlap and thus will have a smaller ratio of areas. Thus, the integral for the free model will be larger, and the probability of that model will increase compared to the scaled model. 


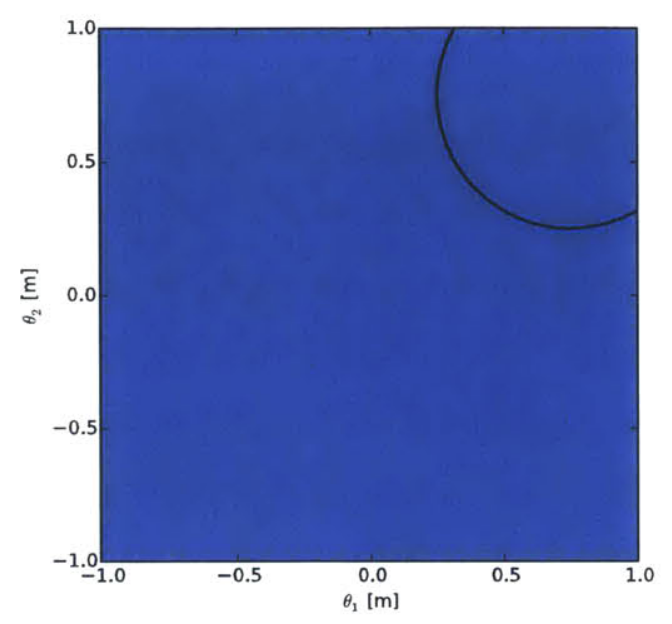

(a) Free - C-space

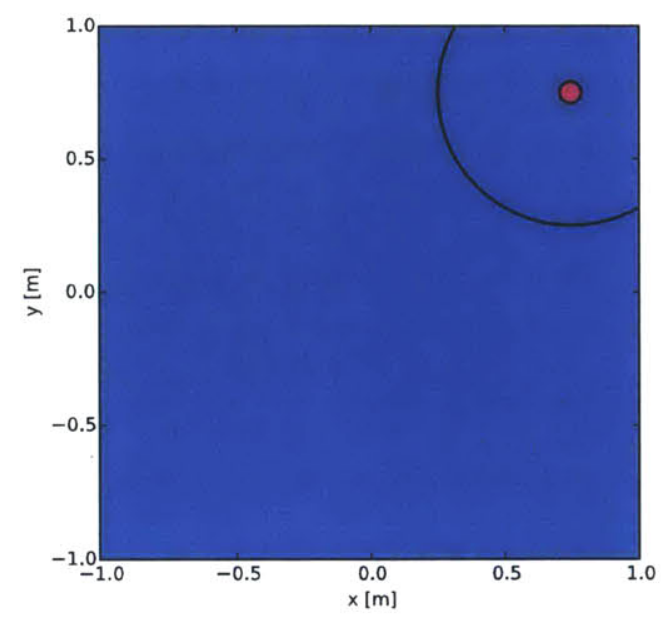

(c) Free - O-space

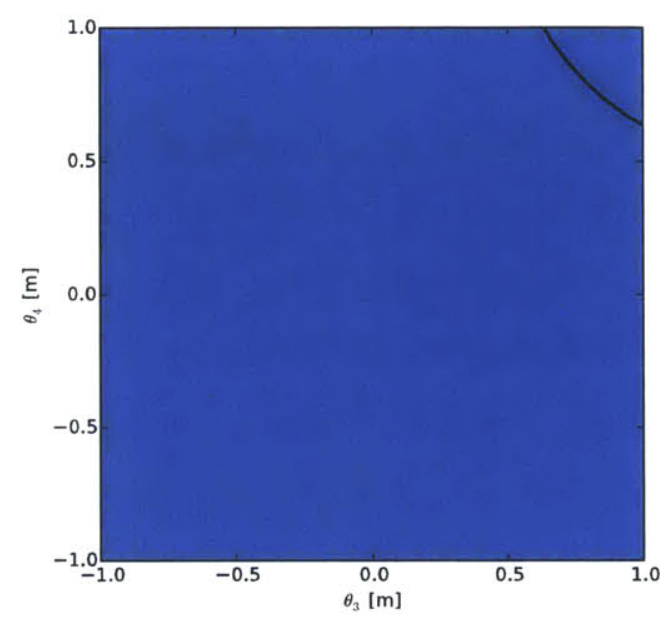

(b) Prismatic - C-space

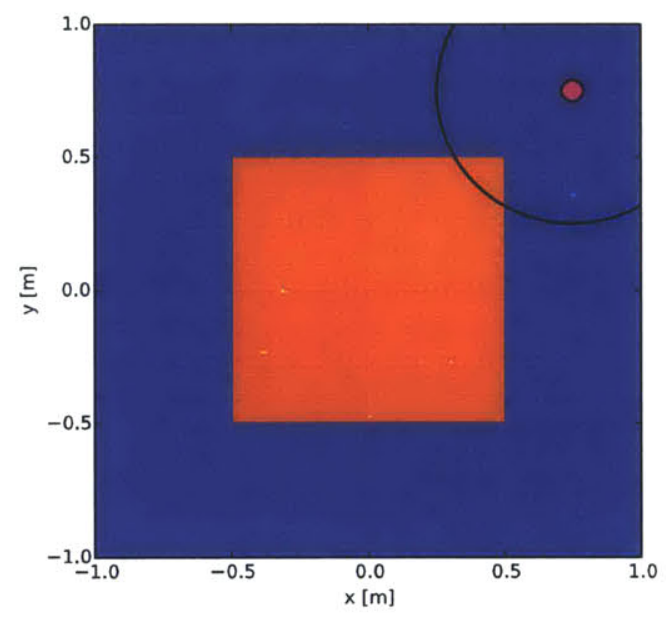

(d) Prismatic - O-space

Figure 4-6: Visual depiction of the projection phenomenon for a scenario of comparing a free hypothesis and a scaled free hypothesis given an observation far from the origin of the observation space. The darkest blue denotes regions of zero probability. The lighter blue denotes regions of lower probability, while orange denotes regions of higher probability. The black circles denote regions where states or their corresponding nominal observations would assign nonzero probability to observation $o$. a) $P(\Theta \mid M)$ shown on the configuration space of the free model. b) $P(\Theta \mid M)$ shown on the configuration space of the scaled free model. c) $P\left(O^{*} \mid M\right)$ shown on the observation space of the free model. d) $P\left(O^{*} \mid M\right)$ shown on the observation space of the scaled free model. 
In summary, the mapping of states to nominal observations affects the inference of a Bayesian filter. Again, this phenomenon is not an error but, instead, follows directly from Bayesian mathematics. Although initially unintuitive, an observation which appears explainable by either model will increase the probability of the model whose probability mass is more concentrated near the observation even if this concentration occurs solely from the configuration to observation space mapping function.

Note that the analysis in this chapter is not specific to a particular belief representation or filtering technique but inherent to Bayesian filtering for model comparison. The results in Chapter 5 exhibit the consequences of these phenomena. 


\section{Chapter 5}

\section{Particle Filter}

This chapter describes the use of a particle filter for state estimation of the kinematic identification problem. As discussed in Chapter 3, the discrete version of the filter suffered from scalability problems. The discrete filter has a time complexity of $O\left(n^{2}\right)$ where $n$ is the number of discrete points, and the number of discrete points we need is exponential in the dimension of the space. This time complexity leads to two problems. First, a space containing high dimensional mechanism models, many parameter sets per model, or many models requires many discrete points for a particular granularity of coverage. If the discretization is too coarse, the estimation can be inaccurate and suffer from misclassification errors. If the discretization is too fine, the filter requires an impractical amount of time to update.

These limitations suggest that solving the full estimation problem requires a much faster approach for a given accuracy. In general, the distributions in the kinematic identification problem will not be well modeled by a single Gaussian or another parametric distribution and the transition and observation models may be non-linear. A discrete Bayesian filter can address this problem but cannot scale. Furthermore, as discussed in Chapter 4, no appropriate continuous state-space, parametric filter is known.

Instead, a particle filter can be used to approximate the distribution through a set of samples with corresponding weights. Much like the discrete filter, the particle filter naturally handles arbitrary distributions and non-linear transition and observation 
models. However, the particle filter has two distinct advantages over the discrete filter. First, a standard particle filter algorithm operates in $O(n)$ time where $n$ is the number of particles. Second, standard resampling techniques for the filter concentrate particles in regions of higher probability which may reduce the number of required particles for the same estimate accuracy. In general, these two properties together drastically reduce the time requirement for the particle filter from that of a discrete filter for the same estimation accuracy. Thus, the particle filter is a natural approach to expand the possible space of mechanisms, complexity of the mechanisms, and space of possible parameters for those mechanisms.

\subsection{Particle Filter Implementation}

This section presents a basic implementation of a particle filter. Thrun, Burgard, and Fox [22] treat the subject more closely and can be referred to for more details including a derivation.

The basic idea behind a particle filter is to represent the belief with a set of samples, called particles, and corresponding weights, called importance weights. This representation allows the filter to approximate arbitrary distributions and easily allows the use of non-linear or piece-wise transitions and observations.

The filter is initialized by sampling a set of particles $\vec{X}$ from the prior distribution. Each particle in this set is given the same weight which may or may not be normalized. In the implementation used, the weights are normalized initially and after each step.

Each update of the filter occurs in three steps. First, for each particle $\vec{x}_{i}$ in $\vec{X}$, a sample is drawn from the the distribution

$$
\vec{x}_{t}^{[m]} \sim p\left(\vec{x}_{t} \mid \vec{a}_{t}, \vec{x}_{t-1}^{[m]}\right)
$$

where $\vec{a}_{t}$ is the action taken at time $t$. We choose this distribution, called the proposal distribution, to be the same as the transition model although, in general, the proposal distribution can be chosen differently to meet different needs. Second, the observa- 
tion $\vec{z}_{t}$ from the world is incorporated by calculating the observation likelihood and updating the importance weight as

$$
w_{t}^{[m]}=p\left(\vec{z}_{t} \mid \vec{x}_{t}^{[m]}\right) w_{t-1}^{[m]}
$$

The particle update occurs each time the robot takes an action and receives an observation.

At each time step, the particles and weights are samples from the belief $b_{t}$. The belief is a continuous distribution over the state while the particle set represents a discrete distribution. Strictly, the cumulative distribution function of the particles approximates that of the belief. Frequently, these two functions converge at $O\left(\frac{1}{\sqrt{n}}\right)$ where $n$ is the number of particles. The discrete set can be used to approximate the continuous belief probability density function by performing kernel density estimation on the particles and weights with an appropriate kernel. However, statistics of the belief may be estimated from the particles directly.

Frequently, a third step, resampling, is implemented. Without resampling from the initial set of particles, many of the particles can potentially have very low weights leaving only a small subset of the original particles to represent the parts of the distribution with substantial probability mass. This problem is called "particle deprivation." One solution can be to have a very large number of particles such that enough always exist in the relevant areas to approximate the belief at a desired resolution. However, often the computation time is the limiting factor of the filter, in terms of performance on a problem or size of the problem instance. Thus, solving the problem by simply increasing the number of particles is infrequently applicable. Instead, resampling the set of particles in a manner which concentrates them in regions of higher probability mass helps maintain enough particles in the areas of interest without requiring an excessive number.

Many resampling techniques exist with various advantages and limitations. A basic resampling technique is to randomly sample particles with replacement from the previous set $\vec{X}_{t}$. A new set of particles $\vec{X}_{t+1}$ of the same size as the previous set 
is used on the next step. The corresponding weights are reset to be equal.

However, as discussed by Arulampalam et al. [1], resampling can also lead to a loss of diversity in the particles. While not resampling at all can lead to a poor estimate of the belief, resampling too frequently can significantly increase computation time and more importantly lead to a scenario where a lack of diversity of particles early on generates a poor estimate of the belief over time. Because resampling often draws samples from the discrete particle set rather than the continuous belief distribution and may sample the same particle more than once, the new set may lack enough variation to represent the belief well. An extreme example, called "particle collapse", occurs when all particles are duplicates which frequently very poorly represents the belief.

For these reasons, one may not want to resample on every iteration. Many ways to decide when to resample exist. A basic method is to calculate the "effective" number of particles left in the filter. The effective number of particles is given by

$$
N_{e f f}=\left(\sum_{i}^{n}\left(w_{k}^{i}\right)^{2}\right)^{-1} .
$$

Given a threshold for the necessary number of effective particles, the filter can automatically choose to resample when necessary to avoid the deprivation problem but not so frequently as to unnecessarily increase computational cost or cause diversity problems.

\subsection{Specific Assumptions for the Given Problem}

\subsubsection{Bank of Particle Filters}

The filtering problem can be stated in two equivalent ways given the previously discussed assumptions of the kinematic identification problem, namely, that mechanisms cannot transition to other mechanism types and that our observation model only depends on the current observation $o_{t}$ and the state $s_{t}$. In general, the state spaces of each mechanism type have different dimensionality. One can filter directly in the 
hybrid space comprised of the mechanism state spaces with a discrete dimension of model type. Equivalently, filtering can occur separately for each model type while normalizations of the distributions to generate a proper PDF consider all filters.

This implementation uses a bank of particle filters, one for each mechanism type. For filter $i, n_{i}$ particles are maintained at each step. Although not necessary, this implementation assumes that the number of sampled particles per filter is equal for all filters and will be referred to simply by $n$. We discuss the ramifications of this choice in Section 4.2. At each time step $t$, the marginal distribution over model types can be calculated by summing the particle weights. Namely, the probability of model $m_{i}$ is

$$
P\left(M=m_{i}\right)=\sum_{j}^{n} w_{j}^{i} .
$$

Below, filter is used to refer to the bank of particle filters.

\subsubsection{Initialization}

The implementation of the particle filter for this problem requires several initialization choices. The filter's initialization reflects our assumptions, and the parameters used are not heavily tuned.

\section{Initial Particles}

When an experiment begins, the origin of the coordinate system used for observations is set to be at the current believed position of the gripper. We assume no noise initially, and thus, in the robot's belief, any particle $\langle m, \theta, x\rangle$ for which $P(o=(0,0) \mid$ $m, \theta, x)=0$ should be discarded, where $\langle m, \theta, x\rangle$ is defined as in Section 1.1.1. That is any initially sampled particle which does not map to the origin of the observation space should receive zero probability and be discarded.

Doing so would be rejection sampling and require a model-specific test for validity to test particles for plausibility. Because only a small subspace of each model's full state space meets this initialization constraint, rejection sampling is very inefficient. 
Instead, hand-built, model-specific samplers are used to generate a set of initial particles. Note that because no parameter set is repeated for mechanisms which can have different parameters, each particle for these mechanism types is a mechanism hypothesis.

\section{Prior Distribution}

Our desired prior distribution is uniform over models. That is

$$
P(M) \sim U\left(m_{0}, m_{m}\right)
$$

Equivalently, the initial probability of each model type is equal. The probability mass associated with each model is distributed evenly among its $n$ particles such that

$$
w_{j}^{i}=1 / n
$$

for all models $m_{i}$.

This prior over models reflects the belief that no model type should be preferred initially over another. Along with the choice, discussed above, to have equal numbers of particles sampled for each model and the constraint that any initial particle must map to a handle pose at the origin, this prior also means initially no parameter set is preferred over another.

Of course, these choices may be modified to reflect other prior information or a different problem's needs. Through the prior distribution, the Bayesian framework can very naturally incorporate information known before the robot's execution of its first action. For example, the robot may be given the type of environment it is in and infer a different distribution over mechanisms based on its environment. Moreover, the robot could receive information, which may color the prior, from a different system or sensor about the particular mechanism it is interacting with before interaction starts. 


\subsection{Model and Filter Parameterization}

Our approach requires choosing a number of parameters. The three main parameters are the covariances of the transition and observation models and the number of particles to sample per filter. We chose the number of effective particles $N_{\text {eff }}=0.5$ as a middle ground and did not tune the parameter.

\subsubsection{Transition and Observation Model Covariances}

Specifying distributions for the transition and observation models in the filter requires choosing appropriate covariance matrices. As in the discrete case, we have limited the covariance matrices to be diagonal. We choose a single standard deviation for all variable dimensions for all mechanism types in the transition model and a standard deviation for both dimensions of the observation model. Two caveats for the transition model is that, first, we desire that the noise added to angles and displacements should be comparable in units of distance because our observation space is a two-dimensional Euclidean plane. To achieve this equality, we scale the noise added to angular dimensions appropriately for the given model and state. Second, when adding noise to the angles, we wrap the values into a specified range to avoid problems associated with aliasing of angular dimensions. We chose the variances on the basis of physical intuition and did not tune them for performance.

Again, as in the discrete case, the filter will undergo testing in simulation and with the real robot. In this case, simulation is a poor testbed for choosing the covariance matrices. In simulation, the robot's interaction with the world is calculated using the same models that the filter's transition model uses. Preliminary experiments with the first four basic models showed very positive results with small transition and observation noise where the noise in the filter matched the noise in the simulated robot interactions.

However, the models are, by necessity, approximate and incomplete when using the real robot. For example, friction, jamming, and other more complicated interaction phenomena are not considered in the models. This choice allows the models to be 
simple and computationally faster but likely leads to inconsistent predictions between the models and the real world. Another main approximation of the models is that, as in the discrete case, the simulated robot is a point robot moving the mechanisms. The full system in the real world is the mechanism connected to a robot arm. Although the simulated point robot acts consistently and symmetrically, the real robot does not, which leads to discrepancies between the predicted and actual transitions.

To examine the impact of model mismatch, we added a bias error to the simulated robot. Specifically, given a predicted nominal state resulting from an action, the simulator would move to a final state only partway to the nominal prediction. For small biases, the filter continued to perform well, but it began to fail frequently as the bias increased. The result demonstrates that the difference between the transition model and robot's interactions can cause errors. Moreover, because the mechanism simulators are not necessarily of equal fidelity, these errors may not be even across model types and may, therefore, skew the results of the filter's inference.

Some of these modeling errors can be effectively modeled as additional noise in the transitions. An appropriate level of noise for the transition model can be determined empirically with the real robot. Separately, experiments with the real robot are also necessary to correctly model the amount of noise in the real robot's observations as many different matched noise levels between a simulated robot and the filter perform well.

For the transition model, robot experiments revealed disagreement between a number of the models and true mechanisms. Often, the predicted transitions were far enough from the actual motions that few particles close to the true mechanism in the state space were likely to have generated the observations. Thus, the filter's belief in the model type of the true mechanism and states corresponding to it drops drastically.

With respect to the particle filter, two different approaches can be taken to address a setting where the transition model accuracy is low relative to the observation model accuracy. Thrun, Fox, and Burgard [23] give a clear explanation of the particle filter and its limitation in this circumstance and suggest various approaches to 
mitigating the problem, including the dual particle filter and a mixture particle filter. Their results suggest that the dual particle filter can also exhibit poor performance, similar to the standard particle filter approach, but that their proposed mixture particle filter can outperform the others. Our implementation avoids the use of a dual or mixture particle filter for two reasons. First and more importantly, in our context, the standard particle filter is easily implemented, requiring the same transition and observation models as our discrete filter. Our observation model requires forward kinematics to compare a state from a given model with an observation. Again, we treat the transition model and the forward kinematics as a black box. The dual particle filter requires the use of inverse kinematics which are more difficult to implement. Also, for sequential mechanisms, such as the latch models, or other complex mechanisms, the inverse kinematics may not be well defined. Second and less crucially, our motivation for moving to the particle filter from the discrete filter is decreasing computational complexity. Our discrete filter required $n=O\left(k^{d}\right)$ points per model where $d$ was the dimension of the model. Then, the discrete filter's time complexity was $O\left(n^{2}\right)$. The particle filter allowed a reduction in the time complexity to $O(n)$. However, a dual or mixture filter likely requires a density estimation step, for example, using a kd-tree, which likely increases the algorithm's time complexity to at least $O(n \log n)$. For these reasons, the chosen solution is adjusting the parameters of the standard particle filter approach for good performance in this problem.

Again, the problem is that the robot's actual transitions does not coincide with the predicted transitions. No adjustment of the noise of these transitions will model this bias. However, a large transition noise can mitigate the issue by stochastically placing some transitioned particles in the area of the observation under the assumption of sufficient particles.

For the observation model, a small variance is accurate for the system. This small noise leads to large differences in observation likelihoods for different states. However, in practice, the filter can perform well with this correctly-modeled variance. No clear motivation exists to increase the observation noise above the true level produced by the robot's sensors. 
The choice of a large transition noise and a small observation noise suggests that we trust the observations much more than the predictions given by models. As explained above, this trust is properly placed as the transition models are poor and the sensor measurements are good.

\subsubsection{Number of Particles}

As discussed in Section 5.1, large numbers of particles should accurately represent the belief but will slow down the filter update. Experimenting with various numbers of particles in simulation aids choosing a particle count that properly balances speed and accuracy.

Early experiments using the first four simple models led to the choice of $n$. For each mechanism type, a "true" mechanism was chosen. 50 experiments of 10 random actions each were run on each mechanism type for varying numbers of particles ranging from 10 per mechanism type to 10,000 . We use a simple distance metric as a measure of error. The metric is a state-space distance adjusted for varying number and type of dimension. The same metric is used for each particle count and, thus, the errors are comparable with each other. The errors given between the best particle in the filter and the true state show the relationship between the number of particles across all four models and average mean-squared error of the filter. Figure 5-1 shows this relationship. The graph shows decreasing marginal improvement in the mean-squared error with small changes between 1,000 and 10,000 particles per model. The graph also shows a decrease in variance over models with increasing particle count. Particle numbers above 10,000 were not tested as moving to 50,000 or 100,000 particles per filter slowed each update of the filter sufficiently such that running the desired number of experiments and actions per experiment required unreasonably long runs.

Without attempting to perfectly tune the particle number for the given problem, we chose 10,000 particles per model for the following simulated and robot results. 10,000 particles seemed sufficiently high to achieve reasonable state estimates while sufficiently low to leave the robot's actual movements, rather than the filter update, as the dominate time expenditure for each step of an experiment. 


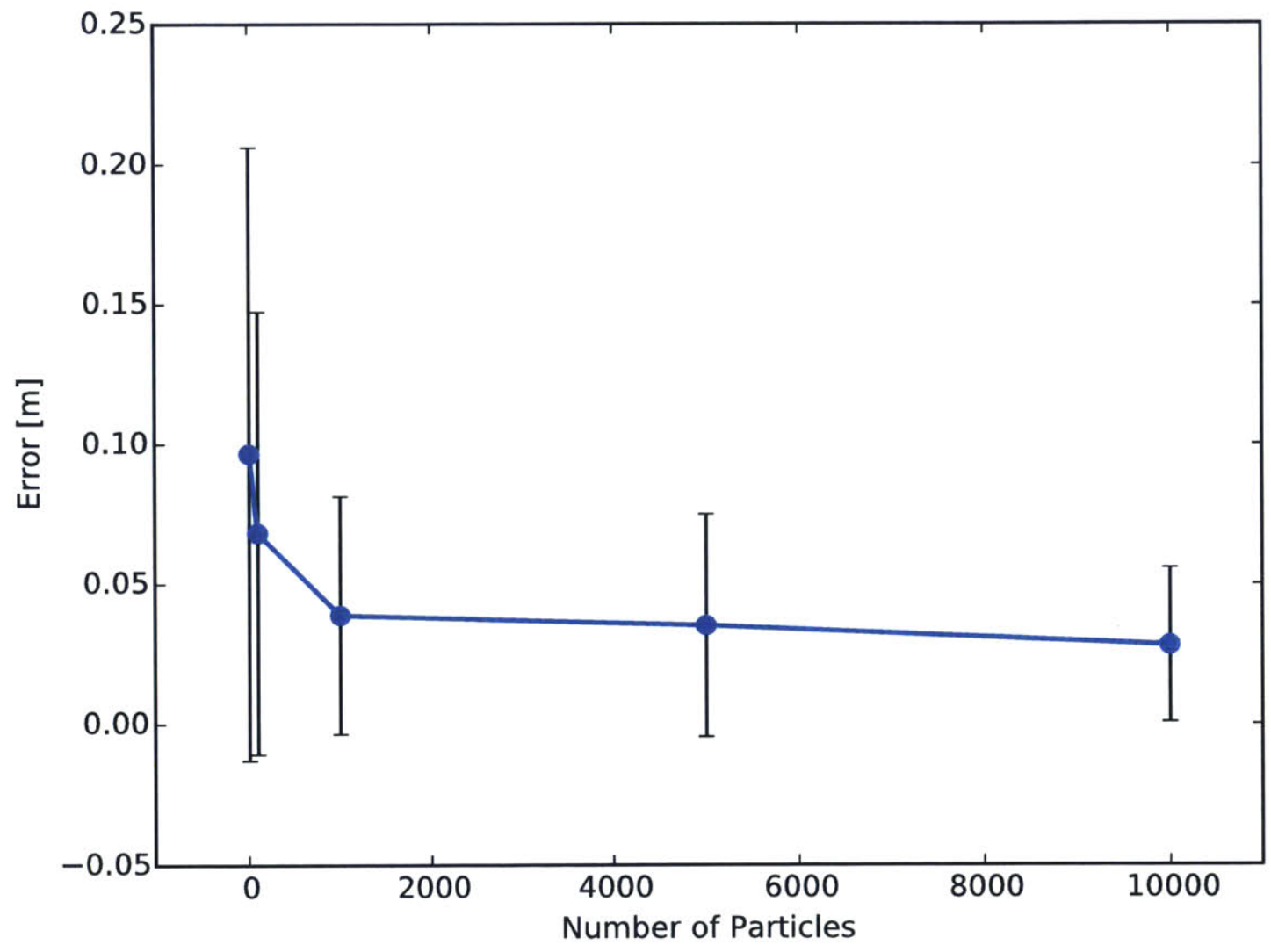

Figure 5-1: The mean-squared error after 10 actions between the true state and best particle in the filter for varying number of particles per model. 


\subsection{Results}

Simulated and real-world experiments demonstrate the performance of the particle filter for the mechanism identification problem. Each experiment runs 10 actions on the mechanism and receives 10 observations from the world. From the belief after the final filter update, the robot determines the type and parameters of the most likely mechanism that the robot is manipulating. In the results below, the particle with the highest weight is used as this model-parameter estimate. This method of choosing an estimate ignores the spatial distribution of the particles. A better estimate could be calculated by finding regions of high probability that may involve multiple particles.

\subsubsection{Evaulation Metric}

Initially, the question of proper classification of mechanism type appeared important. In the discrete case with few, well-spaced hypotheses, determining if the highest probability hypothesis was that corresponding to the true mechanism was nearly a classification problem. In the particle filtering case, with 50,000 hypotheses of the true mechanism in our implementation, which performance metric is appropriate is less clear.

A state-space distance metric is useful to measure error within a mechanism class. Hand-engineering can make these distances somewhat comparable across mechanism types. That distance metric may be sufficient for approximate parameter tuning, such as choosing the number of particles per filter as described above.

However, these metrics do not ask the fundamental question of what the robot will do with the information drawn from its belief. Presumably, the goal of identification is actuation of that mechanism. At a high level, the robot hopes to actuate a mechanism to complete some task. Therefore, a more appropriate metric would involve the robot's ability to actuate the mechanism.

Figure 5-2 depicts a scenario in which misclassification may not be the most appropriate evaluation metric. The robot's observation space is denoted by the axes, and the robot's workspace, denoted by the dashed box, is centered on the origin of 
this space. The straight green line denotes a true revolute mechanism with its handle, denoted as a black dot, at the origin of the observation space. The light green circle denotes the locus of nominal observations for this mechanism. Assume that the robot must actuate the mechanism in the workspace, which will require predicting the mechanism's motion. Compare two possible hypotheses the robot could have of the mechanism. The straight red line denotes a properly-classified revolute hypothesis whose parameters are significantly different than the true mechanism. Again, the light red circle denotes the locus of nominal observations for this estimated mechanism. The light blue line denotes the locus of nominal observations for an improperlyclassified prismatic hypothesis that is tangent to the nominal-observation locus of the true mechanism at the origin. In this example, using the prismatic hypothesis would better predict the motion of the true mechanism in the workspace.

To evaluate the robot's ability to predict the mechanisms motion, one idea would be to measure, in simulation, the disparity between the nominal observations of the estimated state and true state during actuation. For a given experiment, we assume we know the true state $s_{0}^{*}$ at time $t=0$. At the end of a $K$-step experiment, we use the highest-weighted particle from the filter as the estimate $\hat{s}_{K}$ at $t=k$.

As discussed in Section 5.2.2, each experiment begins with the robot's gripper at the origin of the observation space. The true, initial state $s_{0}^{*}$ must map to the origin. However, $\hat{s}_{K}$, the estimated state at the end of the experiment, in general will not map to the origin. We wish to run the same action sequence on both states and, thus, must reset the configuration variables of $\hat{s}_{K}$ to yield a state $\hat{s}_{0}$ that maps to the origin. Note that during this reinitialization, the mechanism type and parameters of the state are not changed.

Given $s_{0}^{*}$, we define a score on $\hat{s}_{0}$ as

$$
\operatorname{score}\left(\hat{s}_{0} \mid s_{0}^{*}\right)=\frac{1}{K} \sum_{k}\left\|\operatorname{OBS}\left(\hat{s}_{k}\right)-\operatorname{OBS}\left(s_{k}^{*}\right)\right\|
$$

where $\operatorname{OBS}(s)$ gives the nominal observation corresponding to state $s$. At each step, we measure the Euclidean distance between the nominal observations corresponding 


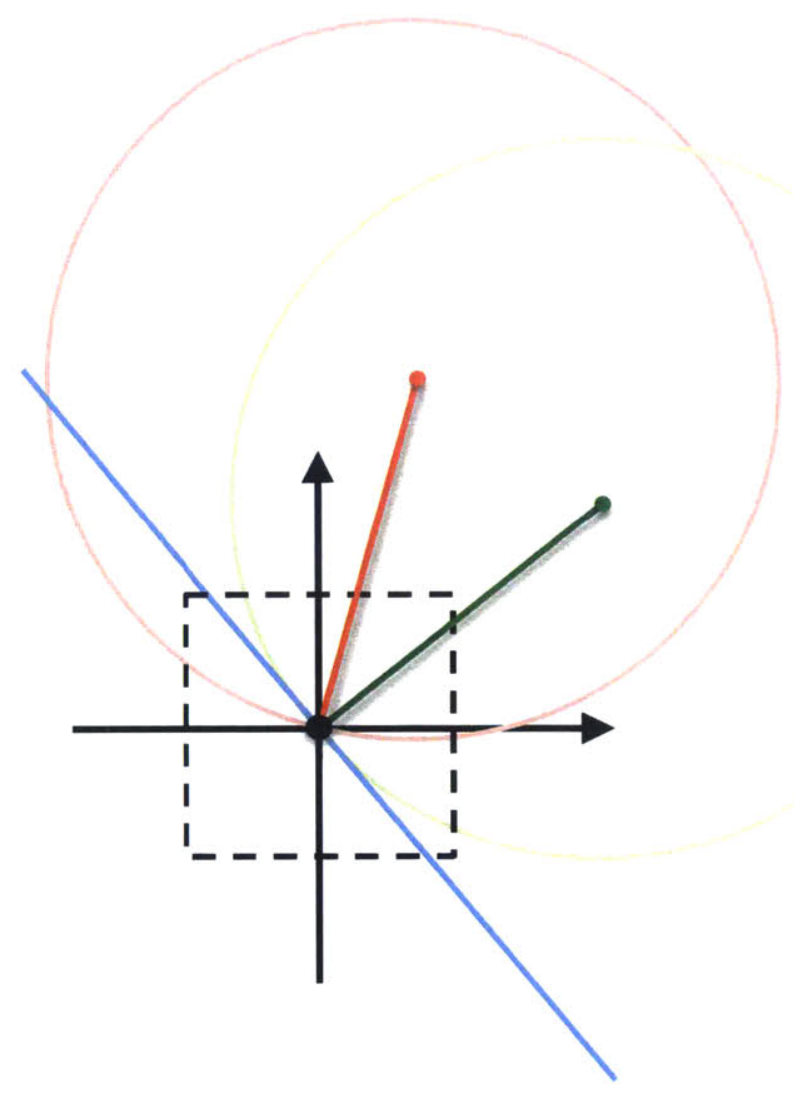

Figure 5-2: The ability to predict the true mechanisms motion may be a more applicable evaluation metric for the mechanism identification problem than misclassification. In this top-down view, the robot's workspace is denoted by a dashed box centered on the origin of the observation space denoted by the axes. A true revolute mechanism is shown by the straight green line with the mechanism's handle at the origin of the observation space. The light green circle shows the locus of nominal observations for this mechanism. The straight red line denotes a properly-classified revolute hypothesis with a corresponding nominal-observation locus shown as a light red circle. The light blue line denotes the nominal-observation locus, tangent to the nominalobservation locus of the true mechanism at the origin, of an improperly-classified prismatic hypothesis. In this example, the prismatic hypothesis would better predict the true mechanisms motions in the workspace. 
to the current estimated and true states. This distance is the error for that action. The average error over all actions in the sequence estimates an actuation-oriented error measure between the estimate and true states.

For the metric, we choose a sequence of actions that covers much of the robot's workspace. Shown in Figure 5-3, this action sequence should actuate all possible mechanisms that this implementation of the filter may encounter. The figure shows the actions in the robot workspace $(x, y)$ as viewed from above. This actuation sequence begins at the workspace origin. The first action is denoted by the lightest blue color while the last action is denoted by the brightest pink color. The first action is in the positive $x$ direction followed by an action in the negative $y$ direction. The actions move clockwise around the workspace. Some actions move towards the origin and back to cover more of the workspace.

This evaluation metric is appropriate because it measures the difference between actuating the estimated mechanism and the true mechanism. Some cases in which the mechanism type is misclassified may have low error in this measure. For example, revolute and prismatic mechanisms can be easily confused if the randomly selected actions do not move the mechanism sufficiently to clearly distinguish a possibly large-radius circle from its tangent line. However, if the robot's ultimate goal is to actuate the mechanism over a certain distance, both of these possible mechanisms may behave similarly in its workspace. The robot could misclassify a revolute joint as a prismatic joint and yet only experience small deviations from its predicted poses when moving the mechanism through its workspace. Alternatively, the robot could correctly classify a mechanism as revolute but estimate its parameters with a large error. Although initially, the classification may seem successful, the poses the robot moves through during actuation may deviate significantly from its prediction. In this case, misclassifying the mechanism to a prismatic joint with more appropriate parameters may provide lower error during actuation than the poorly-estimated but correct-classification case. Thus, a metric involving the quality of prediction of the mechanism's motion seems more appropriate for this problem.

For example, Figure 5-4 shows the result of running the evaluation action sequence 


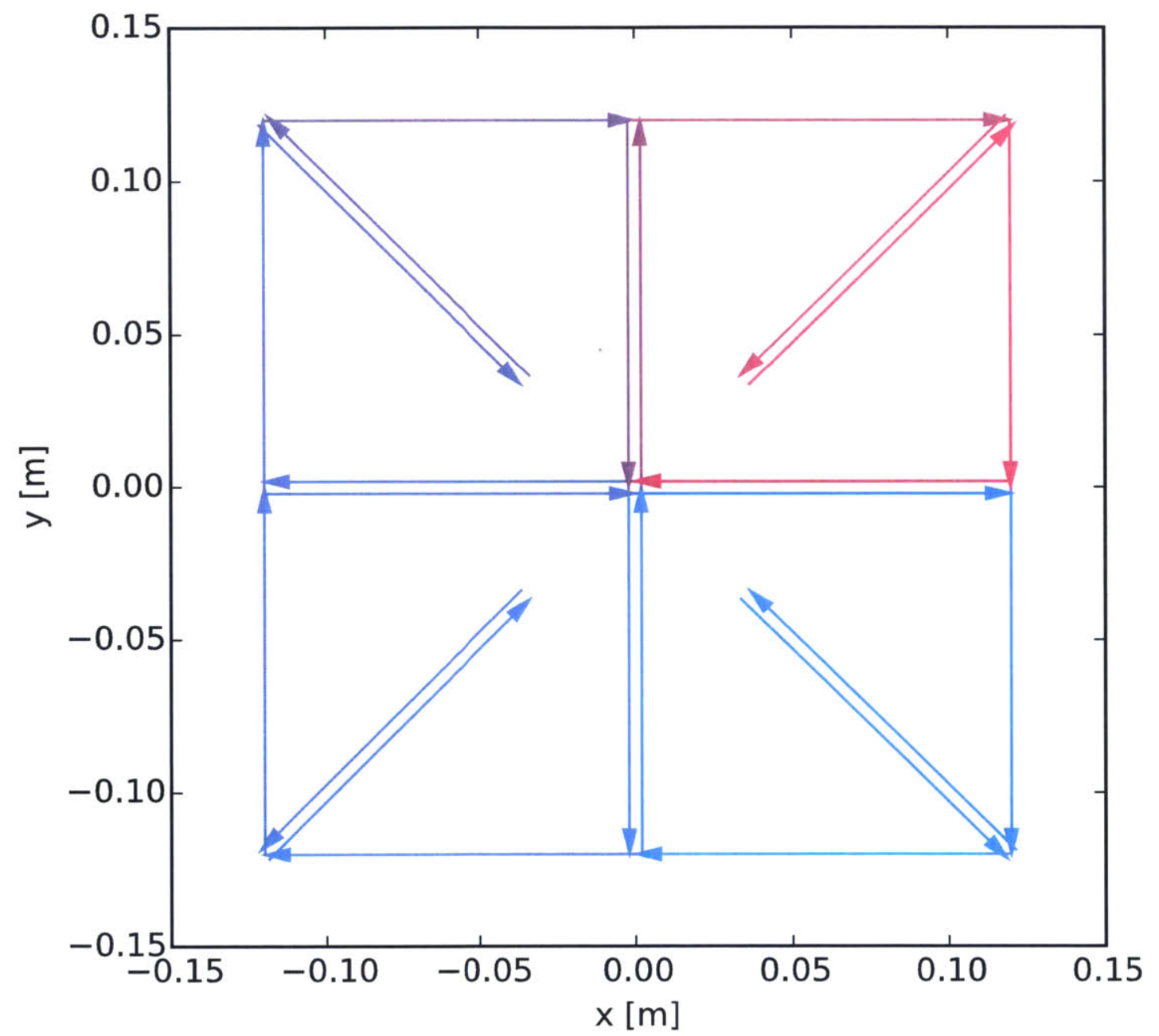

Figure 5-3: Sequence of actions used in the distance metric in the robot workspace $(x, y)$ viewed from above. The action sequence is 24 actions where the first action is denoted by the lightest blue color and the last action is denoted by the brightest pink color. The sequence starts at $(0,0)$, moves in the positive $x$ direction, then in the negative $y$ direction, and continues clockwise around the workspace. 
run on a true revolute joint and an estimated revolute joint. Assuming no noise, the green circle represents all possible nominal observations for the true joint, and the red circle represents the same for the estimated joint. Equivalently, the circles show the legal configuration space of the mechanism projected into observation space. For much of the robot's workspace, the two joints nearly overlap. Noisy observations of the true joint could produce the red joint as an estimate. Shown as colored points, the nominal observations after each action in the evaluation sequence are drawn for each joint overlapping the line designating that joint. The points are colored matching the colors of the corresponding actions shown in Figure 5-3 above. The dashed, blue lines show the distance between nominal observations for the same action on the two joints. Again, the average of this distance over all actions in the sequence is the quality measure of this estimate with respect to the true mechanism. For this action sequence and these two hypotheses, the average prediction distance is $7[\mathrm{~cm}]$. Note that although during experiments the robot's actions are constrained within a 30 $[\mathrm{cm}]$ square centered at the origin, the actions in the evaluation metric are allowed to move the hypothetical mechanisms out of the workspace to allow the same sequence of actions to be used for any state.

To compare, Figure 5-5 shows the result of running the evaluation on the same true revolute joint as above and an estimated prismatic joint tangent at the origin to the true mechanism. The figure is drawn and colored identically to the previous one. With an identical evaluation action sequence, the average prediction distance between these joints is $6[\mathrm{~cm}]$.

As seen in this example, the best-scoring hypothesis may misclassify the joint type and still provide better prediction quality than the highest-probability hypothesis of the correct type. These figures show a simple example of a type of result that was not uncommon during the experiments run in both simulation and on the robot. The discussion of evaluation metric aims to concentrate attention on the filter's ability to predict the result of an action taken and not solely on the filter's ability to correctly classify the mechanism. Of course, the quality of the filter's models of mechanisms limits the quality of the prediction in tasks conducted in the real world. 


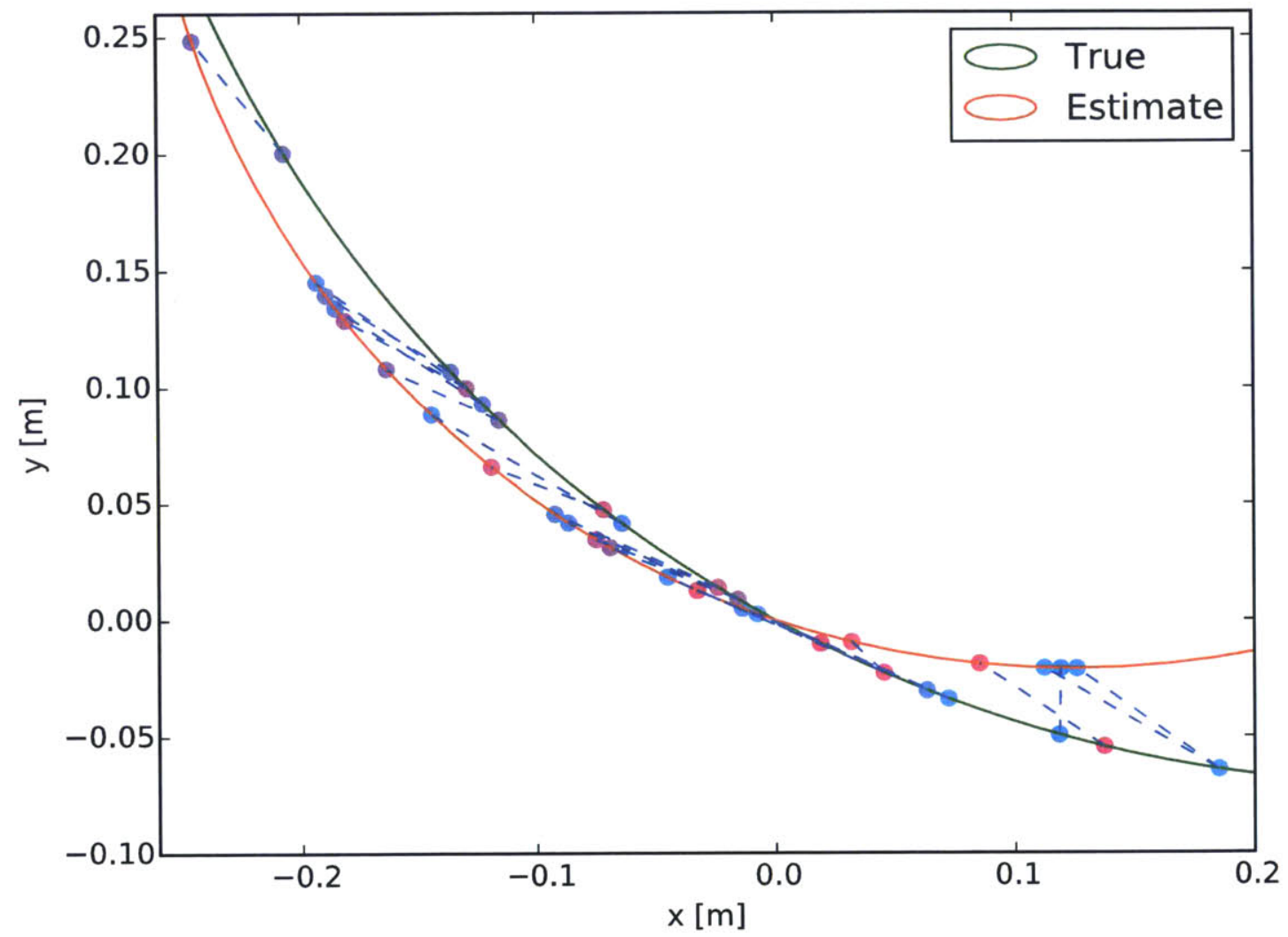

Figure 5-4: Top-down view of the sequence of observations that was generate by the same action sequence, shown in Figure 5-3, on both a true revolute joint, drawn in green, and an estimated revolute joint. The legal configuration space projected into the observation space of the true mechanism is drawn as a green circle, and that of the estimated mechanism is drawn as a red circle. Shown as points, the observations are colored the same as the corresponding actions in the previous figure and drawn on the corresponding mechanism. The dashed blue lines connect corresponding observations and show the distance of the prediction error. The average of these distances is used as the quality metric of the estimate. The average distance between nominal observations for these two mechanisms and initial states is $7[\mathrm{~cm}]$. 


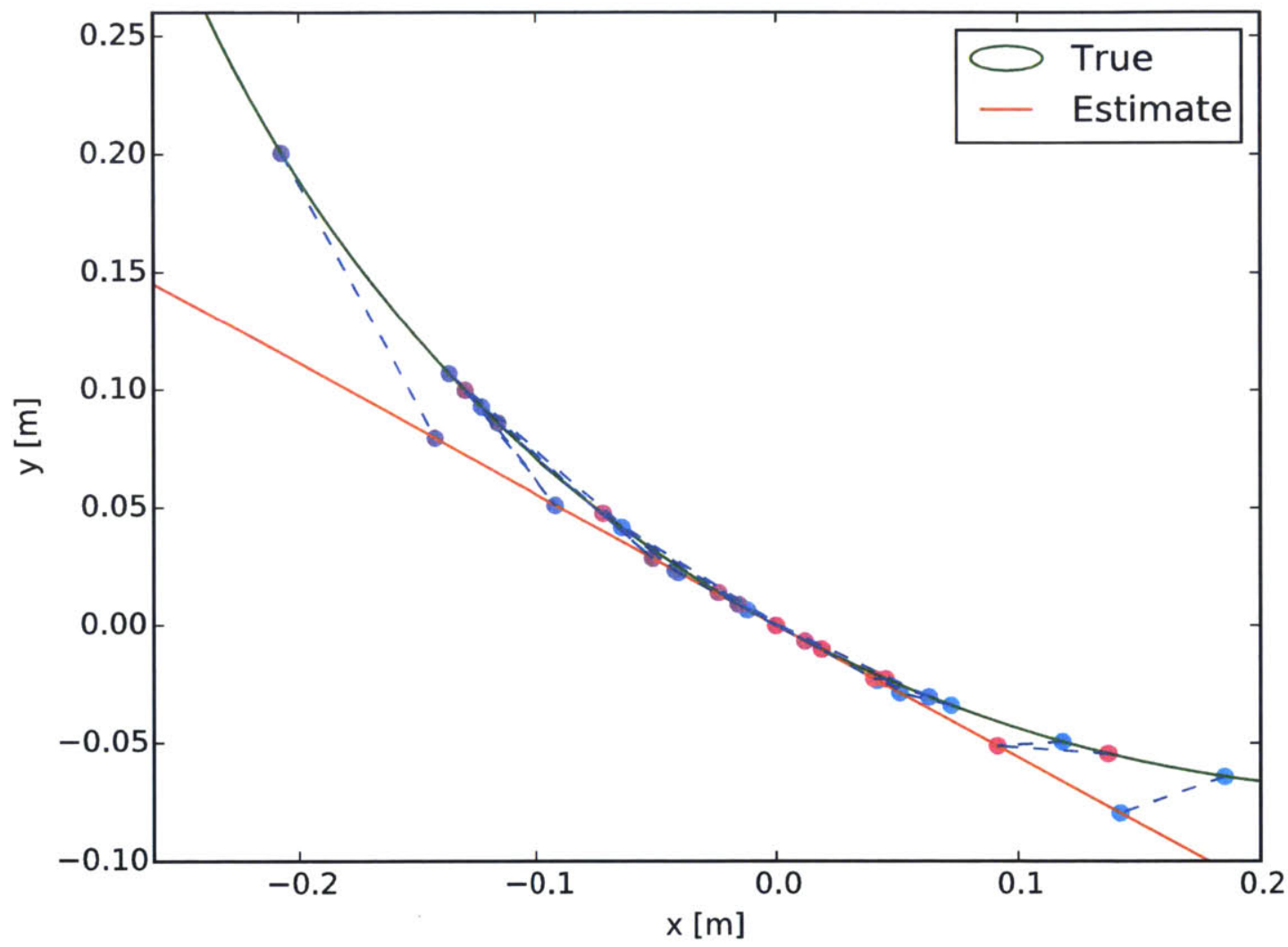

Figure 5-5: Top-down view of the sequence of observations that was generate by the same action sequence, shown in Figure 5-3, on both a true revolute joint and an estimated prismatic joint that is tangent to the true revolute joint at the origin. The legal configuration space projected into the observation space of the true mechanism is drawn as a green circle, and that of the estimated mechanism is drawn as a red line. The figure is drawn in the same manner as Figure 5-4. The average distance between nominal observations for these two mechanisms and initial states is $6[\mathrm{~cm}]$. 
Ultimately, considering this form of evaluation metric returns to the classic balance between exploration and exploitation. In the end, the robot's goal is to actuate mechanisms to perform some higher-level task. Mechanism identification itself can be useful, but identification for the purpose of actuation is the actual task. This evaluation metric, which is independent from the estimation strategy, points to that purpose. However, the robot acts without knowing ground truth. When interacting with a new mechanism instance, choosing a set of actions to gain enough information to make a useful initial actuation choice would appear reasonable. As the robot actuated the mechanism, the system could incorporate more information into the estimate to improve performance. The threshold at which the robot should change from exploring the mechanism to actuating it is a classic design choice.

\subsubsection{Simulation Results}

In simulation, the robot is a point attached to the handle. Generated by the same transition distribution as the filter uses in its transition model, the motions of the simulated robot are modeled on those of the real robot but are much simplified. In simulation, noise is added to the nominal transition of the handle for the "true" mechanism. The standard deviation of this noise is $2.5[\mathrm{~cm}]$ unlike the filter's transitionnoise standard deviation of $10[\mathrm{~cm}]$. As discussed in Section 5.3.1, the large standard deviation in the transition model compensates for deviations between the models and the real world. The world likely does not exhibit such a large noise level. Thus, the noise added to the simulated robot's actions is set at a more realistic level. The standard deviation of the simulated robot's observation model is matched with that of the filter's observation model at $1[\mathrm{~cm}]$ which is representative of the real robot's sensor noise.

To quantify the filter's performance in simulation, a single "true" mechanism for each of the 5 mechanism types underwent 50 experiments of 10 random actions per experiment. The action-sequence evaluation metric described above was used to characterize the quality of the estimate for each experiment. Again, the metric purposefully aims to evaluate the quality of the estimate of mechanism type and parameters 


\begin{tabular}{lc}
\hline Model & Misclassifications \\
\hline Free & 5 \\
Fixed & 0 \\
Revolute & 2 \\
Prismatic & 0 \\
Latch & 6 \\
\hline
\end{tabular}

Table 5.1: Total misclassifications in simulation experiments out of 50 total experiments.

and not simply to classify the mechanism. Also, the metric does not account for the estimation of the configuration of the mechanism after the final action. For completeness, this evaluation also examines the number and type of misclassifications during the experiments.

First, the number and type of misclassifications, a natural metric, are shown in Tables 5.1 and 5.2. The worst performance is while exploring a "true" latch which succeeds in classification in $88 \%$ of the experiments. The confusion matrix shown in Table 5.2 shows a number of important trends. Possible explanations of these trends will be discussed in detail in Section 4.2. First, the free model and latch are frequently confused. These two mechanism types behave similarly in certain configurations of the latch. Second, "true" revolute joints can be confused with prismatic joints. This confusion may result from the small local discrepancy between lines and circles. Third, the fixed and prismatic mechanisms are infrequently, if ever, misclassified. As a brief, approximate explanation, these models can be lower dimensional than other models or spread less in the observation space when particles of these models are transitioned with noise.

Tables 5.3 and 5.4 show the average value, for each model type, of the error calculated by the evaluation metric for the experiments including or not including the misclassifications respectively. First, without including misclassifications, the free and fixed models have exactly zero error. When a free or fixed state is reinitialized, it is identical to the true model by definition as we assume the true mechanism begins in the gripper at the origin. Without including misclassifications, these numbers will always be zero. Next, prismatic estimates generally lead to very small prediction 


\begin{tabular}{|c|c|c|c|c|c|c|}
\hline & \multicolumn{5}{|c|}{ Estimated } \\
\hline & & 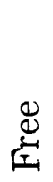 & $\begin{array}{l}\overrightarrow{\widetilde{d}} \\
\text { 运 }\end{array}$ & 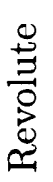 & 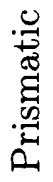 & 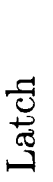 \\
\hline \multirow{5}{*}{ 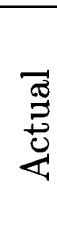 } & Free & 0 & 0 & 0 & 0 & 5 \\
\hline & Fixed & 0 & 0 & 0 & 0 & 0 \\
\hline & Revolute & 0 & 0 & 0 & 2 & 0 \\
\hline & Prismatic & 0 & 0 & 0 & 0 & 0 \\
\hline & Latch & 6 & 0 & 0 & 0 & 0 \\
\hline
\end{tabular}

Table 5.2: Confusion matrix for misclassifications in simulation experiments out of 50 total experiments.

\begin{tabular}{lcc}
\hline Model & Mean $[\mathrm{cm}]$ & SD $[\mathrm{cm}]$ \\
\hline Free & 0.0 & 0.0 \\
Fixed & 0.0 & 0.0 \\
Revolute & 5.2 & 6.8 \\
Prismatic & 0.9 & 0.6 \\
Latch & 9.9 & 5.9 \\
\hline
\end{tabular}

Table 5.3: Error and standard deviation (in $[\mathrm{cm}]$ ) of state estimate using the action sequence evaluation metric over models averaged over 50 simulation experiments without including misclassified estimates.

error, on the order of $1[\mathrm{~cm}]$. Revolute and latch estimates lead to larger errors with a maximum of $10[\mathrm{~cm}]$. We believe these errors are still relatively small considering a estimation angle error of only 0.17 [rad], $9.6^{\circ}$, will produce a handle position error of $10[\mathrm{~cm}]$ for a $60[\mathrm{~cm}]$ radius door.

Including misclassifications and comparing the values to the correctly classified values illuminates a few interesting patterns. First, the values in Table 5.4 will be identical to the values in Table 5.3 if the mechanism type had zero misclassifications. The free, revolute, and latch models all experienced misclassifications. However, the prediction error for these models only increases by a small percentage. The largest change occurs in the free model because it changes from exactly 0 error. These small changes suggest that even estimates of the wrong model type allow the robot to reasonably predict the motion of the "true" mechanism. These results support the use of a actuation-based evaluation metric. Focusing on misclassifications suggests 


\begin{tabular}{lcc}
\hline Model & Mean $[\mathrm{cm}]$ & SD $[\mathrm{cm}]$ \\
\hline Free & 1.4 & 4.7 \\
Fixed & 0.0 & 0.0 \\
Revolute & 5.3 & 6.7 \\
Prismatic & 0.9 & 0.6 \\
Latch & 10.0 & 5.5 \\
\hline
\end{tabular}

Table 5.4: Error and standard deviation of state estimate using the action sequence evaluation metric over models averaged over 50 simulation experiments including misclassified estimates.

a worse performance of the filter. Comparing the evaluation metric values with and without misclassifications suggests that misclassifications only make a small difference in the performance of the filter and perhaps are not paramount as the robot is still able to actuate the mechanism reasonably well even after misclassifying it. Again, as the robot begins to actuate the mechanism after estimating its type and parameters, the filter can use new observations to further refine its prediction.

\subsubsection{Real-robot Results}

The real-world mechanisms were created as much as possible from everyday objects such as desk drawers and cabinets. The robot uses a simple, parallel-plate, two-finger gripper. Because of the size and design of the gripper, the handles of the mechanisms are large to allow the robot to easily grip them and, again, as stated in Section 3.2.2, purposefully do not constrain rotation of the gripper, as the simulations do not model rotational coupling. The robot holds an unconstrained object or no object when interacting with a free model and holds a handle attached to a locked mechanism when interacting with a fixed model. The latch mechanism was hand-built for the robot but is similar to real versions such as a latching gate.

The transition model in the filter does not reflect the actual interaction of the robot with the world. As stated in Section 5.3.1, preliminary experiments with the filter, including simulations where the "true" mechanism's transitions had an added, artificial bias error, showed a decrease in performance as the disparity between the modeled and "true" dynamics increased. Because of the disparity between the models 


\begin{tabular}{lc}
\hline Model & Misclassifications \\
\hline Free & 2 \\
Fixed & 0 \\
Revolute & 4 \\
Prismatic & 0 \\
Latch & 0 \\
\hline
\end{tabular}

Table 5.5: Total misclassifications in simulation experiments out of 50 total experiments.

and the real robot, the robot's performance in the real world using the same filter is likely to be worse than in the simulated world.

To quantify the filter's performance, the robot interacted with objects of all 5 mechanism types. For each of the 5 types, the robot conducted 10 experiments of 10 random actions per experiment. For the revolute, prismatic, and latch mechanism, each experiment explored a mechanism with different parameters to test the filter's performance over different parameter values. However, the robot never explored a mechanism of the same true parameter set more than once. Again, for each experiment, the evaluation metric calculated the quality of the estimate. Similar to the simulation results, the following tables also show the number and type of misclassifications during the experiments.

Table 5.5 shows the total misclassificaitons in the robot experiments. Of the 5 model types, the free and revolute models are sometimes misclassified. Estimating the revolute model has the worst classification performance at $60 \%$. Table 5.6 shows the type of misclassifications for each model. Similar to the simulation results, the filter confuses the free model for the latch but, unlike the simulation results, does not confuse the latch as free. Finally, just as in simulation, the filter misclassifies the revolute model as prismatic.

For the robot experiments, Table 5.7 shows the average error calculated by the evaluation metric for each model. The same trends from simulation appear in the robot experiments. Again, the free and fixed model errors are identically zero. The prismatic model error is small. The revolute and latch mechanism errors are larger than other mechanism types but are small as discussed above for the simulation 


\begin{tabular}{|c|c|c|c|c|c|c|}
\hline & \multicolumn{5}{|c|}{ Estimated } \\
\hline & & 离 & 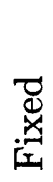 & 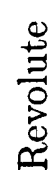 & 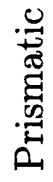 & 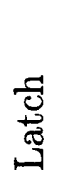 \\
\hline \multirow{5}{*}{ 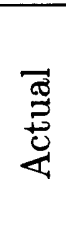 } & Free & 0 & 0 & 0 & 0 & 2 \\
\hline & Fixed & 0 & 0 & 0 & 0 & 0 \\
\hline & Revolute & 0 & 0 & 0 & 4 & 0 \\
\hline & Prismatic & 0 & 0 & 0 & 0 & 0 \\
\hline & Latch & 0 & 0 & 0 & 0 & 0 \\
\hline
\end{tabular}

Table 5.6: Confusion matrix for misclassifications in robot experiments out of 10 total experiments.

\begin{tabular}{lcc}
\hline Model & Mean $[\mathrm{cm}]$ & SD $[\mathrm{cm}]$ \\
\hline Free & 0.0 & 0.0 \\
Fixed & 0.0 & 0.0 \\
Revolute & 13.2 & 10.2 \\
Prismatic & 0.9 & 1.0 \\
Latch & 8.0 & 5.2 \\
\hline
\end{tabular}

Table 5.7: Error and standard deviation of state estimate using the action sequence evaluation metric over models averaged over 10 robot experiments without including misclassified estimates.

results.

Comparing the average error without including misclassifications shown in Table 5.7 with the average error including misclassifications show in Table 5.8 suggest the same result as in simulation. The errors increase by small percentages when including misclassifications which suggests that the robot can reasonably actuate a mechanism even based on a misclassified estimate. In context of our problem, this result reinforces the use of the actuation-based evaluation metric instead of focusing on model classification alone. Furthermore, comparing the values of Tables 5.4 and 5.8 suggest that the filter's performance is similar in simulation and on the robot. The free model performs worse on the real robot although the latch performs better. The revolute model shows the largest change where the errors are much smaller in simulation. Although the absolute value of the error on the revolute model on the robot is small compared to the dimensions of the true mechanism, the increase in 


\begin{tabular}{lcc}
\hline Model & Mean $[\mathrm{cm}]$ & SD $[\mathrm{cm}]$ \\
\hline Free & 2.3 & 4.6 \\
Fixed & 0.0 & 0.0 \\
Revolute & 14.3 & 8.2 \\
Prismatic & 0.9 & 1.0 \\
Latch & 8.0 & 5.2 \\
\hline
\end{tabular}

Table 5.8: Error and standard deviation of state estimate using the action sequence evaluation metric over models averaged over 10 robot experiments including misclassified estimates.

error is significant. Overall, the results suggest that the filter can perform reasonably well in simulation and in robot experiments in the real world with actual common mechanisms.

\subsubsection{Limitations}

The use of the particle filter increased the number of hypotheses from ten in the discrete filter to 50,000 . However, even the particle filter is time-complexity limited. In moving to more complicated models, the number of particles required to cover the state space at the same granularity increases exponentially in the dimension, in general. Although the space addressable by the particle filter is much larger, possible environments that the robot may encounter will easily have too many mechanism types with too high-dimensional state spaces to be handled under reasonable time constraints. Inevitably, the filter must fundamentally be sped up. Possibilities include using a Rao-Blackwellized or Gaussian mixture filter to drastically change the time complexity of belief updates. 


\section{Chapter 6}

\section{Conclusion}

Manipulation can be an extremely useful information-gathering tool. Robots attempting to act robustly in new environments can use many sensor modalities to identify the objects around them. This thesis presents an approach to identifying mechanisms based on manipulation data. The approach uses different versions of a Bayesian filter to estimate the probability of different model types, their parameters, and their variables. The filter utilizes simulations in its transition model which can be modeled in a physics engine or by equations derived by hand or can be represented by data. The robot performs actions, in the form of relative displacements, on different mechanisms. The robot's end-effector positions after these actions serve as the observations. We show in simulation and on our PR2 Robot that our approach can robustly identify and estimate the mechanisms we consider.

\subsection{Reflections}

In Section 1.2, we discuss the motivation for the design choices in our approach. In many cases, these decisions maintained the generality of our approach but made the problem more difficult to solve. Here we reflect on some of the consequences of those choices.

- Not relying on human actuation of the mechanism provides two crucial advantages. First, the robot can reason about the action it attempted, which naturally 
allows us to handle sequential latch mechanisms. Second, this choice allows our approach to be fully autonomous after the robot grasps the mechanism's handle.

- The use of "black-box" models was motivated by our desire not to restrict the form of the models. The mechanism models can be, in principle, hand built, created in a physics engine, represented by data, or learned, parametrically or not, in the world. Avoiding the need for the type of analytical models used in other work may prove crucial when attempting to identify significantly more complicated mechanisms or objects. However, this choice increased the difficulty of our problem. Utilizing a known analytical model provides many directions to simplify the problem or improve performance. The algorithms or even the belief representation could be made specific to the exact mechanisms. Maintaining the "black-box" assumption protected the approaches generality but also increased the difficulty of developing a robust solution.

- Our actions are simple displacements. In principle, the actions could be of many types. The robot could apply forces, instead, or move through trajectories. Depending on the sensors used, the actions may even be distinctly different from gripper motions such as actions to look in a direction or turn on a light. The requirement remains that the model of the mechanism can accept the initial state and whatever action type is used and return a new nominal state.

- Similarly, our choice of observations is not unique. We could measure forces or use visual sensing. The type of sensor used will only affect one part of our approach. For any sensor, the observation model must be able to return the probability of an observation given a state of the world. Our approach allows the use of other sensors and even multiple sensor modalities simultaneously.

- Although any prior may be used, we test the performance of our approach with a uniform prior and show that our strategy can robustly identify and estimate mechanisms. This choice also significantly increases the difficulty of the problem. Many previous works rely on knowing a good initial guess of 
the first direction to move the mechanism. This initial guess is provided by a human, supplied by heuristics using visual data, or from some other method. Having a strong prior on the belief or knowing which action to start with to gain information drastically simplifies the problem of mechanism identification and may not be possible, in general. Starting with a uniform prior in our experiments suggests that our method is robust even without supplied information.

- Similarly, our models of the world are imperfect, which increases the difficulty of developing a robust solution. Perfect models may either be difficult or impossible to create. Our results show that even with imperfect models, the robot perform well in the identification task. Note that if the filter must compare two hypotheses which act very similarly, the quality of the models necessarily must be highly accurate to distinguish the hypotheses. Our approach does not preclude this case, since the "black-box" models may be as precise as needed for the task.

- We chose Gaussian distributions for our transition and observation models. However, if another parametric or nonparametric distribution is more appropriate to model the world, the distributions can be replaced without affecting any other part of the system.

- The phenomena discussed in Chapter 4 strongly promotes the use of good action-selection strategies. Our particle filter performs reasonably well choosing actions randomly but is susceptible to misclassifications when insufficiently informative actions are chosen. Our results in Chapter 3 suggest that choosing actions explicitly to distinguish hypotheses and gain information can significantly improve the performance of the filter.

- In this thesis, we estimate kinematic parameters and configuration variables for the mechanisms. However, the approach is not restricted to these quantities. Future problems may require filtering over other parameters and configurations such as velocities, inertias, stiffnesses, or damping. If these quantities are con- 
tained in the state, the transition and observation models must account from these variables given the action and observation domains. However, Bayesian filtering for estimating the parameters of a mechanism is not restricted to certain types of parameters.

\subsection{Summary of Contributions}

We formulate the mechanism identification problem as a Bayes filter and a discrete filter which correctly distinguishes the mechanism types and parameters among 10 such pairs (Chapter 3). The models include two latching mechanisms types appropriately named as different parts of their configuration spaces provide them different constraints. Furthermore, we compare three action-selection techniques including two active strategies. We show that the myopic entropy-minimizing technique and statistical-racing technique both allow the robot to gather the same information as randomly selecting actions in much fewer steps. We also show that filter updates using the heuristic statistical-racing technique run in approximately the same time as updates using random action selection while the racing strategy provides the same or better performance as the much slower entropy-based strategy. Finally, we show that inevitably the discrete filter will be limited by its time complexity.

We examine Bayesian filtering in a hybrid state space for model comparison and discuss three important consequences (Chapter 4). First, we explain through an example why the belief distribution is unlikely to be unimodal. We believe that the belief may not maintain any standard parametric form in general for this problem domain. We explain by example that the quality of the model for a particular mechanism can affect the inference of the filter. If two mechanism types behave similarly in a portion of the observation space, the probability of hypotheses of the poorly modeled mechanism may decrease even when the robot takes observations for a true mechanism of that model type. We discuss the Bayesian Occam's razor phenomenon in which the filter will assign higher probability to a lower-dimensional mechanism type even if mechanism types of different dimensionality contain states which would 
assign the observation similar likelihoods. Finally, we discuss the projection problem in which the differences in mapping between state and observation spaces for different models also affects the filter's inference. Similarly to the previous phenomenon, we show that the probability of hypotheses for a model type which concentrates its probability near an observation because of its mapping function will increase relative to hypotheses of another type even if both types share the same dimensionality. These phenomena are not restricted to a particular belief representation or filtering technique.

To represent arbitrary distributions and decrease the computational complexity of filter updates, we move to a particle filter implementation. Particle filtering in our hybrid space allows us to expand our state space considered from 10 modelparameter pairs to 50,000 as each initial particle sampled is a different set of model type and parameters. We show in simulation that the filter can correctly classify the mechanism type with high accuracy. More importantly, we show that regardless of classification, the estimate of the mechanism type and parameter set leads to reasonable predictions of motion of the mechanism even when the mechanism type is misclassified. This result is very important given that the robot's ultimate goal is to actuate the mechanism for some purpose and, thus, must be able to predict its motion. Finally, we show that this implementation also works robustly, in terms of not only classification but also motion prediction, on a real-world PR2 robot interacting with common mechanisms.

Overall, we develop robust techniques for identifying mechanisms. We show that with gripper-position information alone, a robot in simulation and in the world can robustly estimate the type and parameters of a mechanism to accurately predict its motion. The results suggest that robots attempting to solve this problem with this approach can act robustly in new environments.

\subsection{Future Work}

Many extensions and new directions exist for this work. Some examples are: 
- In Chapter 3, we explored the affects of active action-selection on the problem. In Chapter 5, we were interested in scaling the state space. An initial next step is to apply active action-selection techniques to the particle filter implementation and explore any differences or consequences arise.

- We restrict our actions and observations to a two-dimensional, horizontal plane. Even given the same action and observation domains, extending these domains to high dimensions can make the approach more realistic for human environments.

- We only use gripper-position observations and displacement actions. Exploring the use of multiple sensor modalities simultaneously or more complex actions could yield significant performance improvements or new, interesting branches of this research.

- At most, we consider six mechanism types, all comprised of rigid bodies, and are only concerned with the kinematic state of the mechanism. The work can be extended to include other, possibly very different, mechanisms or objects as well as estimating other mechanism variables such as friction, inertia, damping, or stiffness.

- Incorporating prior information into the filter from contextual information about the environment or data provided by another system may improve the performance of this approach. This extension would likely be connecting related approaches to initialize this approach rather than changing our filtering strategy itself.

- Inevitably, the filtering techniques presented are not capable of handling very high-dimensional state spaces. Although the particle filter is must faster than the discrete filter, adding more complicated mechanisms with higher dimensionality will eventually make particle updates intractable. Although any technique will eventually succumb to the same curse of dimensionality, extending this work 
to use a Rao-Blackwellized or Gaussian mixture filter could potentially again increase the size of the space our approach can handle. 


\section{Bibliography}

[1] M Sanjeev Arulampalam, Simon Maskell, Neil Gordon, and Tim Clapp. A tutorial on particle filters for online nonlinear/non-gaussian bayesian tracking. volume 50, pages 174-188. IEEE, 2002.

[2] Jan Becker, Christian Bersch, Dejan Pangercic, Benjamin Pitzer, Thomas Rühr, Bharath Sankaran, Jürgen Sturm, Cyrill Stachniss, Michael Beetz, and Wolfram Burgard. The pr2 workshop-mobile manipulation of kitchen containers. In IROS workshop on results, challenges and lessons learned in advancing robots with a common platform, 2011.

[3] Jonathan Brookshire and Seth Teller. Articulated pose estimation via overparametrization and noise projection. In Proceedings of Robotics: Science and Systems $X, 2014$.

[4] Sebastian Hofer, Tobias Lang, and Oliver Brock. Extracting kinematic background knowledge from interactions using task-sensitive relational learning. In ICRA, pages 4342-4347. IEEE, 2014.

[5] Advait Jain and Charles C. Kemp. Pulling open doors and drawers: Coordinating an omni-directional base and a compliant arm with equilibrium point control. In ICRA, 2010.

[6] Advait Jain and Charles C Kemp. Improving robot manipulation with datadriven object-centric models of everyday forces. pages 1-17. Springer, 2012.

[7] Simon J. Julier and Jeffrey K. Uhlmann. Unscented filtering and nonlinear estimation. volume 92, pages 401-422. IEEE, 2004.

[8] Dov Katz and Oliver Brock. Manipulating articulated objects with interactive perception. In ICRA, 2008.

[9] Dov Katz, Yuri Pyuro, and Oliver Brock. Learning to manipulate articulated objects in unstructured environments using a grounded relational representation. In Proceedings of Robotics: Science and Systems IV, pages 254-261, Zurich, Switzerland, June 2008.

[10] Andreas Krause and Carlos Guestrin. Near-optimal observation selection using submodular functions. In $A A A I$, pages 1650-1654, 2007. 
[11] Johannes Kulick, Stefan Otte, and Marc Toussaint. Active exploration of joint dependency structures. In ICRA, 2014.

[12] David JC MacKay. Information theory, inference, and learning algorithms, volume 7. Citeseer, 2003.

[13] Roberto Martín Martín and Oliver Brock. Online interactive perception of articulated objects with multi-level recursive estimation based on task-specific priors. In IROS, pages 2494-2501. IEEE, 2014.

[14] Venkatraman Narayanan and Maxim Likhachev. Task-oriented planning for manipulating articulated mechanisms under model uncertainty. In ICRA, 2015.

[15] Stefan Otte, Johannes Kulick, Marc Toussaint, and Oliver Brock. Entropy-based strategies for physical exploration of the environment's degrees of freedom. In IROS. IEEE, 2014.

[16] Sudeep Pillai, Matthew Walter, and Seth Teller. Learning articulated motions from visual demonstration. In Proceedings of Robotics: Science and Systems $X$, Berkeley, USA, July 2014.

[17] Robert Platt, Leslie Kaelbling, Tomas Lozano-Perez, and Russ Tedrake. Efficient planning in non-gaussian belief spaces and its application to robot grasping. In International Symposium on Robotics Research, 2011.

[18] T Rühr, Jürgen Sturm, Dejan Pangercic, Michael Beetz, and Daniel Cremers. A generalized framework for opening doors and drawers in kitchen environments. In ICRA, pages 3852-3858. IEEE, 2012.

[19] Richard D. Smallwood and Edward J. Sondik. The optimal control of partially observable Markov processes over a finite horizon. volume 21, pages 1071-1088, 1973.

[20] Jürgen Sturm, Advait Jain, Cyrill Stachniss, Charles C. Kemp, and Wolfram Burgard. Operating articulated objects based on experience. In IROS, 2010.

[21] Jürgen Sturm, Cyrill Stachniss, and Wolfram Burgard. A probabilistic framework for learning kinematic models of articulated objects. volume 41, pages $477-526$, 2011.

[22] Sebastian Thrun, Wolfram Burgard, and Dieter Fox. Probabilistic robotics. MIT press, 2005.

[23] Sebastian Thrun, Dieter Fox, and Wolfram Burgard. Monte carlo localization with mixture proposal distribution. In $A A A I / I A A I, 2000$. 